\title{
Effects of bovine pregnancy-associated glycoproteins on gene transcription in bovine endometrial explants
}

\author{
A Thesis
}

Presented to

the Faculty of the Graduate School

at the University of Missouri-Columbia

In Partial Fulfillment

Of the Requirements for the Degree

Master of Science

by

AMANDA L. SCHMELZLE

Dr. Jonathan Green, Thesis Supervisor

July 2021 
The undersigned, appointed by the dean of the Graduate School, have examined the thesis entitled:

Effects of bovine pregnancy-associated glycoproteins on gene transcription in bovine endometrial explants

presented by Amanda L. Schmelzle, a candidate for the degree of Master of Science, and hereby certify that, in their opinion, it is worthy of acceptance.

Dr. Jonathan A. Green

Dr. Michael F. Smith

Dr. Charles R. Brown 


\section{ACKNOWLEDGEMENTS}

First and foremost, I would like to thank my advisor Dr. Jonathan Green for everything that he has done for me in the last several years. Not only did you provide me, a young naïve undergraduate at the time, with an opportunity to start my career in the sciences, but you taught me what it meant to be a good scientist. You are always encouraging me to be curious and to expand my mind. For that, I am so thankful because, without it, I would not have the have the thirst for knowledge that I do today. Your intellect, your problem solving skills, and your eagerness to learn are all qualities that I admire and that I aspire to have as a growing scientist. Most importantly, I am so appreciative of your unending patience with me. Even when my procrastination lead to missed deadlines or when I simply continued to forget to do a task for several weeks, you were patient and simply encouraged me to do my best work. I will be forever grateful of your support and guidance.

I wish to think Dr. Smith for serving as a member on my master's committee but also for being a constant source of support throughout my time at the University of Missouri. Even as a little undergraduate you supported me and taught me things in the lab. As a technician and later as a graduate student you trusted me to teach and help some of your own graduate students. Your trust and support means a lot to me and I am very grateful. I also wish to thank Dr. Brown for serving as a member on my master's committee. You always provided lots of good advice and insight into topics that I would not have thought of. You really helped to guide my reading and thinking of the immunological aspects of this project that I otherwise would have been lost in. You also put up with my bad or boring presentations at lab meetings and I appreciate that!

I would like to thanks my friends and fellow graduate students at MU for being a great support system. A special thanks goes to Ben Nelson for being a great friend and a great source of help in the lab. You saved my butt in the lab on more than one occasion. Play time with your dogs was also a great 
stress relief! Thank you Ben for being a great friend. Lastly, I need to thank my family for being a constant source of love and support throughout not only throughout my graduate program but throughout my entire life. I love you all and appreciate you all very much. 


\section{TABLE OF CONTENTS}

ACKNOWLEDGEMENTS ii

LIST OF FIGURES

LIST OF TABLES

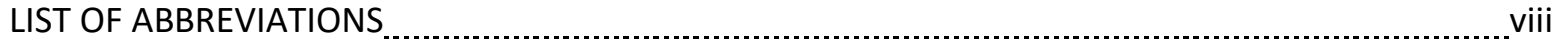

ABSTRACT

\section{CHAPTER}

1. Literature Review

A. Early Embryo Development

i. Blastocyst formation and conceptus elongation

B. Conceptus Attachment and Signaling.

i. Maternal recognition of pregnancy

ii. Apposition and adhesion

1. Adhesion proteins: Mucins

2. Adhesion proteins: Integrins

C. Tissues at the Fetal-Maternal Interface

i. Changes in the epithelia at the interface

ii. Endometrial glands and histotroph in the establishment of pregnancy

iii. Role of MMPs in attachment and placentation

D. Placentation

i. Placental classifications.

1. Gross structure

2. Tissue organization at the placenta-uterine interface

3. Additional placental classifications

E. Binucleate Cells

i. Binucleate cell origin, structure, and differentiation

ii. Binucleate cell function: migration and cell fusion

iii. Products of the binucleate cells

F. Bovine Pregnancy-Associated Glycoproteins

i. Overview

ii. The PAG family

iii. Temporal and spatial expression of PAGs

1. Expression at the interface

2. Use of PAGs in pregnancy diagnosis

iv. Possible functions of the PAGs

1. Matrix remodeling and adhesion

2. Luteotrophic

3. Immunomodulatory

G. Immune System 

i. Overview
ii. Lymphoid cell origins
iii. Myeloid cell origins
iv. Role of chemokines

H. Inflammation, Ovulation, and Pregnancy
i. Role of the immune system during ovulation
ii. Influence of progesterone on immune system
iii. Role of immune system for establishment of pregnancy

2. Effects of bovine pregnancy-associated glycoproteins on gene transcription in bovine endometrial explants
A. Abstract
B. Introductions
C. Materials and Methods
D. Results
E. Discussion 80

APPENDIX

1. The use of a monoclonal antibody to rapidly purify bovine pregnancy-associated glycoproteins
A. Summary
B. Materials
C. Methods
D. Notes

2. Sandwich ELISA for the detection of PAGs
A. Summary 91
B. Materials
C. Methods 92
D. Notes

3. SDS-polyacrylamide gel electrophoresis (PAGE) for detection and evaluation of bovine cotyledonary PAG extracts
A. Summary.
B. Materials
C. Methods 95
D. Notes 96

4. Assessment of changes in gene transcription of MMPs and prostaglandin synthases by real time PCR in bovine endometrial explants exposed to PAGs
A. Summary
B. Materials
C. Methods
D. Notes

5. Caspase-3 and PCNA IHC for assessment of apoptosis in endometrial explants exposed to PAGs
A. Summary 100
B. Materials 100
C. Methods 101
D. Notes 102 


\section{LIST OF FIGURES}

Figure $\quad$ Page

1. Representative images of the microvillar junction in the ewe .....................................................10

2. Pictorial illustrations of gross placental structures …....................................................................20

3. Pictorial illustrations of placental types based on histological structure ......................................21

4. Pictorial illustrations of fetal-maternal interdigitation types .........................................................24

5. Representative patterns of PAG immunolocalization in bovine placentomes ..............................31

6. Transcript abundance of chemokines in endometrium of nonpregnant heifers after

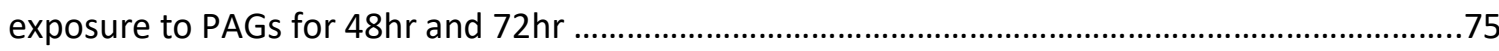

7. Transcript abundance of chemokines in endometrium of pregnant heifers after exposure to PAGs for $48 \mathrm{hr}$ and $72 \mathrm{hr}$

8. PAG-treated vs nontreated transcript abundance of chemokines in endometrium of nonpregnant heifers after 48 and $72 \mathrm{hr}$

9. PAG-treated vs nontreated transcript abundance of chemokines in endometrium of pregnant heifers after 48 and $72 \mathrm{hr}$

10. H\&E staining of nontreated and PAG-treated endometrial explants

11. Analysis of the purification of PAGs from cotyledonary extract by SDS-PAGE and Sypro Red staining

12. Transcript abundance of MMPs in endometrium of nonpregnant and pregnant heifers after exposure to PAGs for $48 \mathrm{hrs}$ and $72 \mathrm{hrs}$

13. Transcript abundance of prostaglandin synthase genes in endometrium of nonpregnant heifers after 48 and $72 \mathrm{hr}$

14. Transcript abundance of prostaglandin synthase genes in endometrium of pregnant heifers after 48 and $72 \mathrm{hr}$

15. Immunofluorescent staining for cellular apoptosis and proliferation in endometrial explants exposed to PAGs for 24 and 96 hours 


\section{LIST OF TABLES}

Table

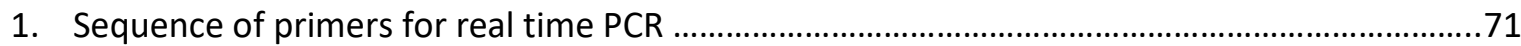




\section{LIST OF ABBREVIATIONS}

\begin{tabular}{|c|c|}
\hline Abbreviation & $\underline{\text { Meaning }}$ \\
\hline $\mathrm{CL}_{\text {. }}$ & Corpus luteum \\
\hline TE & Trophectoderm \\
\hline $\mathrm{LE}$ & Luminal epithelium \\
\hline IG & Integrin \\
\hline IFN. & Interferon \\
\hline $\mathrm{IFN \tau} \ldots .$. & Interferon-tau \\
\hline MUC1.... & Mucin-1 \\
\hline ECM & Extracellular matrix \\
\hline SPP1 & Secreted phosphoprotein $1 /$ Osteopontin \\
\hline GE & Glandular epithelium \\
\hline RGD & Arg-Gly-Asp \\
\hline MRP. & Maternal recognition of pregnancy \\
\hline ESR1 & Estrogen receptor alpha \\
\hline CG. & Chorionic gonadotropin \\
\hline $\mathrm{LH}_{\ldots} \ldots \ldots$ & Luteinizing hormone \\
\hline OXY & Oxytocin \\
\hline PGF2 $\alpha$ & Prostaglandin F2 $\alpha$ \\
\hline PGE2 & Prostaglandin E2 \\
\hline OXTR & Oxytocin receptor \\
\hline BNC. & Binucleated cells \\
\hline UGKO & Uterine gland knockout \\
\hline ECM $\ldots . .$. & Extracellular matrix \\
\hline MMP & Matrix metalloproteinase \\
\hline EnJSRN .... & Endogenous Jaagsiekte sheep retrovirus \\
\hline Env. & Envelope \\
\hline Berve-A & Bovine endogenous retrovirus envelope element-like transcript-A \\
\hline PAG $\ldots \ldots \ldots \ldots \ldots$ & Pregnancy-associated glycoprotein \\
\hline
\end{tabular}




\begin{tabular}{|c|c|}
\hline $\mathrm{PL}$ & Placental lactogen \\
\hline PSPB. & Pregnancy-specific protein B \\
\hline PSP60 & Pregnancy-specific protein of sixty thousand molecular weight \\
\hline boPAG & Bovine pregnancy-associated glycoprotein \\
\hline ELISA & Enzyme-linked immunosorbent assay \\
\hline GCP2. & Granulocyte chemotactic protein-2 \\
\hline CXCL6 & C-X-C motif chemokine ligand 6 \\
\hline CXCL5. & $\mathrm{C}-\mathrm{X}-\mathrm{C}$ motif chemokine ligand 5 \\
\hline ELR & Glutamate-Leucine-Arginine \\
\hline SERPINA14 & Uterine serpin \\
\hline NK. & Natural killer cells \\
\hline $\mathrm{TCR}_{\ldots}$ & T-cell receptor \\
\hline$B C R$ & B-cell receptor \\
\hline HSC & Hematopoietic stem cell \\
\hline PLC. & Protein lipase C \\
\hline $\mathrm{MCP}$ & Monocyte chemoattractant protein \\
\hline MIP... & Macrophage inflammatory protein \\
\hline
\end{tabular}




\title{
EFFECTS OF BOVINE PREGNANCY-ASSOCIATED GLYCOPROTEINS ON GENE TRANSCRIPTION IN BOVINE ENDOMETRIAL EXPLANTS
}

\author{
ABSTRACT \\ Pregnancy-associated glycoproteins (PAGs) are a complex gene family, whose members are \\ expressed by trophoblasts of ruminants and related species. In cattle, the PAGs accumulate at the \\ trophoblast-uterine interface and many can enter the maternal circulation. However, very little is \\ known about the role they play in pregnancy although preliminary results suggest that PAGs at the \\ placenta-uterine interface play roles involving matrix turnover and immune modulation. This study was \\ designed to provide further insight into the biological roles of bovine PAGs by measuring changes in \\ endometrial transcript abundance for some matrix metalloproteinases (MMPs) and \\ chemokines/cytokines. PAGs for these experiments were purified from mid-gestation bovine placental \\ extracts by affinity chromatography. Heifers were synchronized and bred by artificial insemination with \\ high fertility semen $(n=14)$ or dead semen $(n=5)$. Heifers were slaughtered at day 18 post- \\ insemination and the reproductive tracts were obtained and flushed to determine if a conceptus was \\ present. Endometrial explants were collected and split between 4 groups: pregnant with and without \\ $15 \mu \mathrm{g} / \mathrm{ml} \mathrm{PAG}(\mathrm{n}=10)$ and nonpregnant with and without $15 \mu \mathrm{g} / \mathrm{ml}$ PAG $(\mathrm{n}=9)$. Endometrial explants \\ were cultured with or without added PAGs for up to 96 hours at $37^{\circ} \mathrm{C}$ and $5 \% \mathrm{CO}_{2}$ and samples were \\ harvested at 24 hour intervals for extraction of RNA and fixation. This study focuses on the 48 and 72 \\ hour collection points. Transcript abundance for target genes was analyzed in the endometrial tissue by \\ quantitative PCR. The normalization control transcript was peptidylprolyl isomerase A (PPIA). After 48 \\ and 72 hours, significant increases in CXCL1, CXCL2, and CXCL5 as well as MMP1, MMP3 and MMP13 \\ were measured in the PAG-treated endometrium from both pregnant and non-pregnant animals \\ $(\mathrm{P}<0.05)$. CCL11 was upregulated at both time points in the pregnant endometrium but only after 72 \\ hours in the nonpregnant endometrium. There were also significant decreases in message for CCL2, \\ CCL8 and CCL16 in the PAG-treated groups from both pregnant and non-pregnant animals at each time
}


point $(\mathrm{P}<0.05)$. Significant decreases in $C X C L 10, C X C L 12$, and Regakine message were seen only in PAGtreated endometrium from pregnant animals $(P<0.05)$. Structural differences in the luminal and glandular epithelium were seen in the PAG-treated biopsies from both non-pregnant and pregnant heifers. These results suggest that PAGs are capable of inducing structural changes as well as changes in transcript abundance in bovine endometrial explants, which suggests that this model system might be useful to assess PAG function at the placenta-uterine interface. 


\section{CHAPTER I}

\section{LITERATURE REVIEW}

\section{Early Embryo Development}

\section{Blastocyst formation and conceptus elongation}

In cattle, the morula stage embryo enters the uterus 4-5 days after mating (2.5-3 days in sheep). By days 7-8 the blastocyst has formed and on days $9-11$ in cattle and days 7-8 in sheep, hatching from the zona pellucida occurs (Guillomot 1995; Hafez 1993). Because ruminant trophoblasts do not degrade or invade the maternal endometrium, it is believed that the ruminant conceptus compensates for a lack of direct access to maternal blood by occupying as much surface area as possible to increase the amount of exchange that can take place. Toward these ends, the embryo undergoes considerable expansion wherein it transforms from a $3 \mathrm{~mm}$ spherical/tubular conceptus to a $250 \mathrm{~mm}$ filamentous conceptus between days 13-17 in cattle (days 11-14 in the sheep). By day 18 (day 14 in sheep) the conceptus completely occupies the full length of the uterine horn ipsilateral to the corpus luteum (CL) and even extends into the contralateral horn (Guillomot 1995; Hafez 1993; Senger 2005; Spencer et al. 2004). Day 19 and day 15 after mating in cattle and sheep, respectively, marks the end of the pre-attachment phase and the beginning of the apposition phase. The conceptus will continue to elongate, however, until at least day 21 and day 16 in cattle and sheep, respectively.

\section{Conceptus Attachment and signaling}

\section{Maternal recognition of pregnancy}

Pregnancy maintenance requires that progesterone levels remain elevated beyond the length of a typical estrous or menstrual cycle. This means that the CL must be maintained throughout the entire pregnancy or until such time when the placenta can provide enough progesterone to support the 
pregnancy. For this to occur, the conceptus must signal its presence to the maternal system to prevent luteal regression. This process is known as maternal recognition of pregnancy (MRP; Spencer et al. 2004a). MRP signaling from the conceptus usually falls into two categories: 1) luteotropic, i.e. directly stimulating the $\mathrm{CL}$ to maintain luteal function and 2) anti-luteolytic, i.e. prevention of luteolytic signals such as prostaglandin $\mathrm{F}_{2 \alpha}$ (PGF2 $\alpha$; Bazer et al. 2009). Human and primate embryos produce chorionic gonadotropin (CG) as a luteotropic signal. CG is similar to luteinizing hormone (LH) and it can bind to the same receptors on the $\mathrm{CL}$, thereby promoting luteinization rather than luteolysis ( $\mathrm{R}$. Michael Roberts, Xie, and Mathialagan 1996; Fazleabas 2007; Fuller W. Bazer et al. 2009). Rodents maintain the $\mathrm{CL}$ through mating-induced release of prolactin from the anterior pituitary; this mechanism maintains the $\mathrm{CL}$ until about day 12 of pregnancy at which point placental lactogens from trophoblast giant cells take over the role (Soares 2004; Fuller W. Bazer et al. 2009). In pigs, the exact signal for MRP is unclear. It has long been accepted that conceptus-derived estrogens act as the primary MRP signal (Geisert et al. 1987 and 2015) by diverting PGF2 $\alpha$ secretion away from the uterine vasculature and into the uterine lumen (Bazer and Thatcher 1977). However, recent data suggest that conceptus estrogens are necessary but not sufficient for pregnancy recognition and establishment (Meyer et al. 2019).

In ruminants, the signal for MRP is interferon-tau (IFNT), a Type-1 interferon secreted by the trophoblasts of the peri-attachment conceptus (Helmer et al. 1987; Imakawa et al. 1987; Roberts et al. 1992; Roberts 2007). IFNT Mrna and protein expression in the blastocyst is low but starts to increase around the time of conceptus elongation. Around day 14-15 in cattle, and day 12-13 in sheep, Mrna expression rises dramatically accompanied by an increase in protein production. The dramatic rise in IFN $\tau$ expression is probably due to the proliferation of the trophectoderm during conceptus elongation. This allows for large accumulations of IFNt to interact across a large surface area of its likely target tissue (Ealy and Yang 2009). One study reported that a single filamentous ovine conceptus could produce up to 500ng of IFNt per hour (Ashworth and Bazer 1989). This increase in expression is also significant 
because it is occurring at the time in which MRP is required for pregnancy to be establish. In cattle, this is around day 16 and conceptus IFNT Mrna expression peaks around day 15-16 (Ealy and Yang 2009).

At the end of a normal ruminant estrous cycle when a viable conceptus is not in the uterus, the $\mathrm{CL}$ is regressed via a positive feedback loop between oxytocin (OXY) and $P G F_{2 \alpha}$. OXY, initially released from the posterior pituitary, induces the secretion of uterine $\mathrm{PGF}_{2 \alpha}$ from the uterus. The PGF $\mathrm{F}_{2 \alpha}$ enters the uterine venous drainage from where it quickly enters the ovarian artery via a counter-current exchange mechanism arising from the arrangement of the uterine venous and ovarian arterial vasculatures. The $\mathrm{PGF}_{2 \alpha}$ binds to its receptor on luteal cells, which induces the release of oxytocin from them. The oxytocin circulates and binds to its receptors on the uterus to induce a new pulse of PGF $F_{2 \alpha}$. The $\mathrm{PGF}_{2 \alpha}$ arising from this positive feedback loop induces a series of responses that suppress progesterone production from luteal cells and induce the functional demise of the corpus luteum. IFNT acts as an anti-luteolytic signal by downregulating the expression of estrogen receptor $\alpha$ (ESR1) in the endometrium. This change decreases estrogen-induced OXY receptor (OXTR) expression. Without OXTR expressed in the endometrium, OXY cannot induce the pulsatile release of luteolytic $\mathrm{PGF}_{2 \alpha}$ from the endometrium and thus prevents regression of the CL (Ealy and Yang 2009). IFNt may also prevent luteolysis through another mechanism. At high concentrations, IFN can increase production of $\mathrm{PGE}_{2}$ from bovine endometrial epithelial and stromal cells (Asselin, Bazer, and Fortier 1997). PGE 2 has long been regarded as a luteotrophic and luteoprotective prostaglandin (Shelton et al. 1990). Therefore, it is not unreasonable to infer that IFNז may be both inhibiting luteolysis and promoting luteinization for sustained CL function. Overall, there is strong evidence to support the hypothesis that IFN $\mathrm{\tau}$ is a crucial factor in the recognition and establishment of pregnancy in ruminants. 


\section{Apposition and Adhesion}

The initial stage of implantation, when the trophectoderm (TE) of the growing conceptus begins to closely associate with the uterine luminal epithelium (LE), is called the apposition phase. The apposition phase is marked by close association between the endometrial LE and the conceptus TE, loss of anti-adhesive components of the uterine glycocalyx, such as mucins, and subsequent unmasking of underlying adhesion proteins, such as integrins, to promote conceptus attachment to the LE. At this stage several morphological changes are also occurring within the LE and TE including cytoskeletal reorganization, microvilli formation, and epithelial interdigitation. The apposition phase is followed by the adhesion phase where more stable interactions are made between the two epithelia, which results in the TE becoming more fully adhered to the endometrial LE. This is mediated by adhesion proteins and interdigitation of endometrial and chorionic villi. This begins around day 21 in cattle and day 16 in sheep (Guillomot 1995; Spencer et al. 2004).

\section{Adhesion proteins: Mucins}

The anti-adhesive properties of the endometrial glycocalyx are due in part to the apical expression of mucins, primarily MUC1 and MUC4. Mucins are heavily glycosylated surface glycoproteins that are present on the surface of mammalian mucus membranes throughout the body, including the female reproductive tract. MUC1 is a main mucin found in several mucosal epithelia in mammals; among its many known functions, it also has been shown to play a crucial role in conceptus-uterine interactions. Mucins contain highly extended extracellular domains that can project $200-500 \mathrm{~nm}$ from the apical cell surface. By comparison, typical cell surface adhesion proteins, such as integrins, may only extent 10-30 nm from the cell surface (Thathiah and Carson 2002). MUC1 expression in the uterine LE is normally high prior to apposition and attachment. The abundance of MUC1 prevents the embryo from interacting intimately with other cell surface receptors. However, under the influence of progesterone 
in pigs and sheep, MUC1 expression decreases, which then allows the conceptus to interact with integrins and other adhesion proteins on the LE surface (Johnson et al. 2001; Thathiah and Carson 2002). In sheep, uterine LE MUC1 protein is very abundant on the apical surface from Days 1-7 of the estrous cycle. Expression then begins to decline though Day 15 of the estrous cycle. This decline in MUC1 expression correlates with increasing progesterone levels and the onset of implantation. Furthermore, MUC1 expression on the LE of pregnant sheep is very low and is barely detectable by Day 17 of pregnancy when intimate contacts between the LE and conceptus are being made (Johnson et al. 2001). A similar expression pattern has also been seen in pigs (Bowen, Bazer, and Burghardt 1996).

MUC1 expression in the uterus is regulated by ovarian steroid hormones. In mice, MUC1 expression is greatly stimulated by estrogen while progesterone appears to antagonize the actions of estrogens (Surveyor et al. 1995). Ovariectomized gilts treated with either estrogen, progesterone, or estrogen + progesterone showed high MUC1 immunostaining of the uterine LE in response to estrogen. However, in the presence of progesterone (with or without estrogen) MUC1 staining was barely detectable (Bowen, Bazer, and Burghardt 1996). Treatment of mice with antiprogestins restored MUC1 expression (Surveyor et al. 1995) and inhibited embryo implantation (Vinijsanun and Martin 1990). Collectively, these data demonstrate that loss of MUC1 on the uterine LE is required for embryo/conceptus implantation (DeSouza et al. 2000; Thathiah and Carson 2002).

\section{Adhesion proteins: Integrins}

As mucins and other anti-adhesive components of the endometrial glycocalyx are altered via steroid hormone regulation and/or the actions of local or blastocyst-derived proteases, the underlying adhesive proteins are unmasked. These alterations allow adhesive proteins on the LE and TE to interact. Integrins (IGs) are a family of heterodimeric transmembrane glycoproteins that facilitate cell-cell and cell-extracellular matrix (ECM) adhesion as well as mediate intracellular signaling, cellular 
differentiation, and cell motility (Y. Takada, Ye, and Simon 2007). IGs are present on both the uterine LE and the conceptus TE and are thought to play a crucial role in adhesion of these two epithelia during implantation. For example, null mutations in IG subunits $\alpha_{v}, \alpha_{5}, \beta_{1}$, or $\beta_{5}$ in mice lead to implantation failure, failure of the chorion and allantois to associate, and embryo lethality (Hynes 1996). Some species show spatial and/or temporal regulation of IGs in the endometrium throughout the estrus cycle, suggesting regulation by ovarian steroid hormones. Pigs constitutively express $\alpha_{v}, \alpha_{3}, \alpha_{4}, \alpha_{5}, \beta_{1}$ and $\beta_{3}$ subunits on the apical LE throughout the estrous cycle. However, $\alpha_{4}, \alpha_{5}$, and $\beta_{1}$ show spatial and temporal regulation (Bowen, Bazer, and Burghardt 1996). In cattle, $\alpha_{3}, \alpha_{4}$, and $\beta_{1}$ are constitutively expressed in the endometrium while expression of $\alpha_{6}$ and the dimer $\alpha_{v} \beta_{3}$ changes throughout the cycle (Kimmins and MacLaren 1999). In contrast to cattle, sheep do not appear to modify temporal or spatial expression of IGs in the endometrium. Variations in IG expression among these closely related species suggests differences in implantation mechanisms (Kimmins and MacLaren 1999; Johnson et al. 2001).

Often, similar IGs are expressed on both the endometrial LE and conceptus TE. In pigs and sheep, IGs $\alpha_{v}, \alpha_{4}, \alpha_{5}, \beta_{1}, \beta_{3}$, and $\beta_{5}$ are expressed on both the LE and TE around the time of conceptus attachment (Bowen, Bazer, and Burghardt 1996; Johnson et al. 2001). Similar IG expression may suggest common ligands, potentially serving as bridging molecules for conceptus adhesion. Osteopontin (Secreted phosphoprotein 1/SPP1) is one such bridging molecule. SPP1 is a secreted phosphoprotein of the endometrial glandular epithelium (GE) and is a component of the uterine histotroph. Studies in pigs (Garlow et al. 2002), sheep (Johnson et al. 2001; Johnson, Burghardt, Joyce, et al. 2003), and cattle (Kimmins, Lim, and MacLaren 2004) have shown that SPP1 protein increases in the endometrium and/or in uterine flushing around the time of implantation and may remain high throughout pregnancy. This is notable because SPP1 contains an Arg-Gly-Asp (RGD) sequence motif that mediates binding to integrins such as $\alpha_{v} \beta_{1}, \alpha_{v} \beta_{3}, \alpha_{4} \beta_{1}, \alpha_{4} \beta_{5}$, and $\alpha_{5} \beta_{1}$, some or all of which have been identified in the endometrium of domestic animal species (Bowen, Bazer, and Burghardt 1996; Johnson et al. 2001; Johnson, Burghardt, 
Joyce, et al. 2003; Thomas E. Spencer et al. 2004; Fuller W. Bazer et al. 2010; Johnson, Burghardt, and Bazer 2014; Johnson, Burghardt, Bazer, et al. 2003). Taken together, these data suggest that SPP1 could serve as a bridging ligand between IGs on the endometrial LE and the conceptus TE to facilitate conceptus adhesion and implantation. Other potential IG bridging molecules that could mediate or facilitate conceptus implantation include GlyCAM-1 (Giblin et al. 1997; Hwang et al. 1996; Thomas E. Spencer et al. 2004), galectin-15 (Kimber and Spanswick 2000; C. A. Gray et al. 2004; Thomas E. Spencer et al. 2004), and ECM components such as fibronectin, vitronectin, and laminin (Johansson et al. 1997; Burghardt et al. 2002; Wennerberg et al. 1996; Bowen, Bazer, and Burghardt 1996). These observations are suggestive that multiple adhesion mechanisms are involved in implantation and the establishment of a successful pregnancy.

\section{Tissues at the fetal-maternal interface}

Conceptus attachment not only involves changes in cell surface protein expression but also requires structural changes in the endometrium and TE to facilitate subsequent processes associated with placentation. Humans, primates, and rodents have an invasive type of placentation that requires extensive endometrial remodeling, such as LE erosion, stromal decidualization, and vascular remodeling (Enders and Blankenship 1999; Fazleabas 2007; Furukawa, Kuroda, and Sugiyama 2014). Ruminants and pigs exhibit a non-invasive type of implantation and placentation where little, if any, of the LE is lost. Instead, an extensive network of villous interdigitation can be seen between the LE and TE as well as vascular and glandular remodeling. The following sections will discuss these topics within the context of ruminant attachment. 


\section{Changes in the epithelia at the interface}

The epithelial changes that occur during conceptus attachment are similar between ruminant species, the main differences are associated with the timing of such events. The following section will discuss the timing of events in relation to bovine pregnancy.

As discussed previously, during apposition the LE and TE become closely associated with each other. This is accommodated by a reduction of the apical microvilli along the surface of the TE. Unlike in other species, however, loss of endometrial microvilli does not appear to occur in ruminants (Guillomot et al. 1981; Guillomot et al. 1982; Spencer et al. 2004). Prior to the start of apposition (around day 12 of pregnancy in cattle), the intercaruncular (described later in 'Placentation') LE starts to develop large, bulbus cytoplasmic protrusions that resemble the 'pinopods' observed in rodents. The function of these cytoplasmic protrusion is not well understood, unlike rodents, however, they are not predicted to perform pinocytotic functions like those of rodents. It's thought that they may, instead, play a role in exocytosis and secretion of endometrial products during early pregnancy for support of the growing conceptus (Guillomot et al. 1986). This also occurs in cyclic animals, however, when no conceptus is present the protrusions regresses (Guillomot and Guay 1982). Around the start of apposition (about day 18 in cattle) the caruncular (described later in 'Placentation') LE begins to take on a folded appearance. Simultaneously, the conceptus trophoblasts that are normally rounded and covered in microvilli along their apical surface start to lose their surface microvilli and take on a more spindle-like shape. Their surface becomes irregularly rigid and perhaps slightly folded (Guillomot, Fléchon, and Wintenberger-Torres 1981; Guillomot and Guay 1982; Wooding, Staples, and Peacock 1982). By day 20 , when firmer contacts are beginning to be made between the LE and TE, the TE is completely devoid of microvilli. This process appears to happen sequentially along the length of the conceptus starting in the TE closest to the embryo because non-adherent trophoblasts in the uterine 
horn contralateral to the $\mathrm{CL}$ are still covered in microvilli at the same stage (Guillomot and Guay 1982; M. Guillomot 1995).

As apposition progresses and the two epithelia are pulled closer to each other, the microvillar surface of the uterine LE presses against the TE which gives the appearance of trophoblast projections into the 'crypts' between the uterine microvilli (Figure 1A). The rigid, folded surface of the TE also accommodates the invagination of the TE into the uterine microvillar crypts (Lawn, Chiquoine, and Amoroso 1969; Guillomot, Fléchon, and Wintenberger-Torres 1981). Localized areas of the TE begin to proliferate and form large columns made exclusively of trophoblasts known as 'papillae'. These trophoblastic papillae form in the intercotyledonary regions of the TE and project deep into the uterine glands (Figure $1 \mathrm{~B}$ and $\mathrm{C}$ ). It's thought that this is meant to aid in adhering the conceptus to the uterine LE by acting as an anchoring point. The papillae could also be privileged sites of endocytosis of glandular secretions to supply the peri-attachment conceptus with histotrophic nutrition (Guillomot and Guay 1982; Wooding, Staples, and Peacock 1982). These structures are short-lived, however, and start to degenerate around the start of conceptus adhesion.

Another characteristic change in the uterine epithelium of ruminants during attachment is the formation of a fetal-maternal syncytium. The syncytium is formed from the migration of a unique trophoblast cell type known as a giant binucleate cell (BNC). BNCs differentiate from mononucleate trophoblasts. They first appear in the TE between day 18 and 20 and shortly thereafter begin to migrate across the fetal-maternal interface (Wooding and Wathes 1980; Wooding 1982b). When BNCs cross the interface into the uterine LE, they fuse with a uterine epithelial cell to create a hybrid trinucleate cell. The trinucleate cells can continue to fuse with migrating BNCs until a large multinucleated syncytium is formed. These syncytial masses seem to only occur within the caruncular regions. In the cow, the syncytium is only transiently present and is usual gone by day 40 of pregnancy. However, in the sheep 
and goat the syncytium is present throughout pregnancy. More on BNCs and syncytial formation will be discussed later in this review.

Figure 1 


\section{Endometrial Glands and histotroph in the establishment of pregnancy}

Due to the non-invasive properties of the ruminant conceptus and the prolonged preattachment period, the nutrition for the early conceptus is through endometrial secretions known as 'histotroph'. Uterine histotroph contains substances that are essential for the growth and development of the fetus. The main tissues responsible for producing histotroph are the uterine LE and glands.

Uterine glands are a very important feature of the endometrium. They serve many important roles in the uterus during pregnancy including 1) synthesizing, secreting, or selectively transporting histotrophic substances into the uterine lumen (Bazer 1975); 2) promoting conceptus elongation by producing embryotrophic factors (Simintiras, Sánchez, McDonald, and Lonergan 2019c); and 3) assisting in blastocyst attachment as discussed previously (Wooding 1982a). Uterine gland development, or adenogenesis, begins postnatally in most domestic animals. It involves the differentiation and budding of the LE, which forms the GE, followed by invagination, coiling, and extensive branching throughout the uterine stroma (C. A. Gray, Bartol, et al. 2001). These steps are mediated by intrinsic factors such as changes in gene expression, cell proliferation, and cell-cell interactions as well as extrinsic factors such as pituitary and ovarian hormones (Kelleher, DeMayo, and Spencer 2019). Progesterone and estrogen are known to be especially important in endometrial adenogenesis. Blocking estrogen action with prolonged exposure to progesterone from birth to puberty creates a uterine gland knockout (UGKO) model, i.e. an animal that has substantially fewer than normal glands (Bartol et al. 1997; C. A. Gray et al. 2000; Filant, Zhou, and Spencer 2012). UGKO ewes are completely infertile. When bred, they carry morphologically normal blastocysts up until the time of attachment, at such time conceptus growth becomes retarded (Gray, Taylor, et al. 2001; Gray, Bazer, and Spencer 2001; Gray et al. 2002). This would suggest that the failure of the conceptus to elongate is due to the absence of embryotrophic factors originating from the uterine glands (Gray, Bartol, et al. 2001; Spencer, Kelleher, and Bartol 2019). 
What are the embryotrophic factors in the uterine histotroph? Currently, a single embryotrophic factor cannot be determined. This is most likely due to the fact that the uterine histotroph is incompletely defined and because of its dynamic properties (Bazer 1975; Gray et al. 2001a; Mullen et al. 2012; Spencer et al. 2019). It is likely that there is no single embryotrophic factor, rather a dynamic heterogeneous mixture of growth factors, macromolecules and enzymes that supports growth and development of the trophoblasts. In addition to the composition of uterine histotroph itself, the composition of the secreted material is constantly altered by enzymes that are present (Simintiras et al. 2019c; Simintiras et al. 2019d) Not unexpectedly, histotroph production and secretion is regulated by steroid hormone actions. Current research suggests that the influence of progesterone alters histotroph composition to promote membrane biogenesis (Simintiras et al. 2019a), amino acid metabolism (Simintiras et al. 2019b), as well as glucose and nucleotide metabolism (Simintiras et al. 2019c). All of these data support the theory that uterine histotroph supports and/or promotes conceptus elongation by supplying the building blocks, metabolic energy and growth stimulates needed for trophoblast proliferation.

\section{Role of MMPs in attachment and placentation}

The morphological changes occurring during early pregnancy that have been discussed here all involve remodeling of the extracellular matrix (ECM). To support the attaching conceptus, and later the growing placenta, a delicate balance of degradation and formation of the extracellular environment must be regulated. This is done largely through the regulation of a family of enzymes known as the matrix metalloproteinases (MMPs). The MMPs are a gene family of more than 26 enzymes that are broadly grouped based on their preferred substrates (e.g. collagenases, gelatinases, stromelysins) or unique expression patterns (membrane-type-MMPs) (Birkedal-Hansen et al. 1993; Nagase and Woessner 1999; M. F. Smith et al. 2002). They are zinc- and calcium-dependent enzymes that are responsible for the degradation of the proteinaceous components of the ECM such as collagen I, II, III, 
and IV, fibronectin, gelatin, and laminin (M. F. Smith et al. 2002). Remodeling of a tissue relies on the regulation of these enzymes at the level of transcription, through activation of the latent enzymes, and by directly inhibiting proteolytic activity of the mature enzyme (Smith et al. 1999; Smith et al. 2002). Most MMPs are secreted as proenzymes and require further processing from other enzymes such as serine proteinases (L. L. Espey and Lipner 1994), mast cell proteinases (Suzuki et al. 1995), and by other MMPs (Lijnen et al. 1998; Ruangpanit et al. 2002; Ra and Parks 2007)However, some MMPs are activated prior to secretion by the serine protease furin. These include MMP-11, MMP-23, and all membrane-type (mt-) MMPs (Strongin et al. 1995; Cao et al. 1996; Nagase and Woessner 1999; Sato et al. 1999). The activated enzymes are predominantly regulated by a small family of inhibitors known as tissue inhibitors or metalloproteinases (TIMPs $-1,-2,-3$, and -4 ). TIMPs bind noncovalently to the catalytic domain of most activated MMPs with high affinity and a 1:1 stoichiometry (Nagase and Woessner 1999; M. F. Smith et al. 2002). Contrarily, it is predicted that TIMP-2 is involved in the activation of proMMP-2 in vivo (Z. Wang, Juttermann, and Soloway 2000).

Regulation of tissue remodeling is primarily dependent upon the ratio of MMPs and their inhibitors (M. F. Smith et al. 2002). For species with invasive implantation, MMPs are especially important in aiding of degradation of the LE as well as the surrounding matrix to accommodate the invading trophoblasts (Hulboy 1997). During conceptus attachment, MMPs are expressed in the bovine endometrium and the TE throughout pregnancy. In particular, MMP-2 and -9 have been shown to play a role in attachment/implantation in most species (Harvey et al. 1995; Menino et al. 1997; Salamonsen, Nagase, and Woolley 1995; Franek, Salamonsen, and Lopata 1999; Jeziorska et al. 1996). In ruminants and pigs, TIMPs are also produced by both the TE (Menino et al. 1997; Hirata et al. 2003) and the endometrium (Salamonsen, Nagase, and Woolley 1995). They are thought to be acting to limit the invasive activity of the TE by limiting MMP activity (R. M. Roberts, Xie, and Trout 1993; Menino et al. 1997; Salamonsen, Nagase, and Woolley 1995). The ruminant endometrium has the capacity to produce 
a range of MMPs. Of note, MMP-1, $-2,3,-9$, and -14 have been shown to be expressed by the endometrium during attachment (Salamonsen, Nagase, and Woolley 1991; Salamonsen et al. 1993; Walter and Boos 2001). The endometrium also secretes TIMP-1 and -2 (Hampton et al. 1995). MMP-2 may be the most abundant of these MMPs and it is often colocalized with MMP-14 and TIMP2, both of which are involved in the activation of proMMP-2 (Z. Wang, Juttermann, and Soloway 2000; Uekita et al. 2004; Hashizume 2007). The TE also secretes MMP-2 and -9 as well as TIMP-2. Interestingly, TIMP-2 is very abundant within BNCs. This perhaps demonstrates a regulatory function of the BNCs in matrix remodeling (Hirata et al. 2003). There is also a lot of vascular remodeling occurring in the endometrium and in the chorion during conceptus attachment. MMPs are known to play a large role in vascular remodeling by degrading the endothelial basement membrane and remodeling the surrounding ECM to allow endothelial migration (Rundhaug 2005). During pregnancy, MMP-2 and -9 are localized around blood vessels in the caruncular and intercaruncular endometrium as well as in the chorion (Walter and Boos 2001). This would suggest a role for these MMPs in vascular remodeling during pregnancy in ruminants. Though we've been able to observe changes in endometrial ECM components, remodeling of the connective tissue, and vascular remodeling during attachment and placentation in ruminants, the exact mechanism by which this occurs is still unclear (M. Guillomot 1995; 1999; Bairagi et al. 2016).

Matrix remodeling is a continuous process in the endometrium and the placenta throughout pregnancy. However, there is a pause in matrix remodeling prior to parturition (Gross, Williams, and Russek-Cohen 1991). During this time MMPs increase in the TE, most likely to begin the process of utero-placental separation (Dilly et al. 2011). An increase in trophoblastic apoptosis is also seen in the final stage of pregnancy. Degradation of the placental ECM by MMPs is not followed by ECM deposition, which leads to apoptosis of the trophoblasts and eventually detachment of the placenta and fetal membranes at the time of parturition (Boos, Janssen, and Mulling 2003). 


\section{Placentation}

\section{Placental classifications}

Many centuries of evolution has allowed mammals to develop a unique way to retain and sustain the fertilized egg through all necessary stages of development until birth (R Michael Roberts, Green, and Schulz 2016). This is accomplished through the development of a chorioallantoic placenta in a subset of animals called the Eutherians (placental mammals). In his monograph on comparative placentation, Mossman defined the placenta as an "apposition or fusion of the fetal membranes to the uterine mucosa for physiological exchange" (as cited by Roberts, Green, and Schulz 2016). He went on to describe the process of placentation as "an approximation or combination of an embryo's tissues with those of its natural or surrogate parent for physiological interchange" (as cited by Allen C Enders and Blankenship 1999). From these definitions we can take away two key concepts: 1) the placenta is derived from both fetal and maternal tissues, 2) these tissues must form close interactions to facilitate appropriate nutrient exchanges for a successful pregnancy. In particular, the placenta facilitates exchange of water, nutrients, and gasses; in addition, it eliminates fetal metabolic waste (R Michael Roberts, Green, and Schulz 2016). Due to the importance of these functions, it might be assumed that the placenta would be structurally conserved among eutherian mammals. This, however, is not the case. There are many morphologically distinct placental variations between mammalian species, but there are also several shared characteristics. This diversity among placental structures created a need for classification systems. Two particularly useful classification systems that were developed were based on 1) gross placental structure and 2) the cell layers present at the interface (P. Wooding and Burton 2008; Furukawa, Kuroda, and Sugiyama 2014). Other placental characteristics can be use in 
addition to these criteria such as whether any tissue (fetal or maternal) is lost at parturition and whether any accessory placental structures happen to be present (P. Wooding and Burton 2008).

\section{Gross structure}

The gross structure of the placenta refers to the area of fetal-maternal exchange. More specifically, it refers to the distribution of villi or folding between the endometrium and the chorion where exchange takes place. It defines whether that area of exchange is found over the entire chorionic surface or is restricted to certain specialized regions (P. Wooding and Burton 2008; Furukawa, Kuroda, and Sugiyama 2014).

(1) Diffuse - A diffuse placenta is characterized by folds/villi that are present over the entire chorionic surface. These vascularized villi are in contact with nearly the entire luminal surface of the endometrium (Figure 2A). This placental structure is most notably found in pigs and horses.

(2) Cotyledonary - A cotyledonary placenta is characterized by localized areas of vascularized chorionic villi known as 'cotyledons' that intercalate into aglandular regions of uterine endometrium known as 'caruncles' (Figure 2B). Together, the combined structure consisting of a cotyledon and a caruncle is a 'placentome' (G. J. King 1993). Depending on the species there could be up to 150 placentomes on a single placenta (P. Wooding and Burton 2008). This type of placenta is found in ruminant ungulates.

(3) Zonary - A zonary placenta is characterized by a restricted region of complex chorionic folding that forms a band wrapped around the middle of the conceptus (Figure 2C). This creates an equatorial 'zone' of fetal-maternal exchange, hence the name 'zonary'. This placental structure is most notably found in carnivores. 
(4) Discoid - This type of placenta is characterized by chorionic villi that are restricted to one or more distinct discs (Figure 2D), hence the name 'discoid' (P. Wooding and Burton 2008; Furukawa, Kuroda, and Sugiyama 2014). This placental structure is found in many species, most notable in rodents, lagomorphs and higher primates.

\section{Tissue organization at the placenta-uterine interface}

While classifying a placenta based on gross structure is simple, it does not provide much functional insight into how physiological exchange takes place. Grosser was the first to propose a more relevant classification system based on the number of intact cell layers at the fetal-maternal interface (Grosser 1909; 1927; referenced by Mossman 1987 and Amoroso 1952). This approach is still considered one of the most useful and instructive methods for functionally describing placental types. Grosser developed four placental categories that were essentially describing the number and type of maternal tissue layers that were apposed to the chorion in the mature placenta. These categories were: epitheliochorial, syndesmochorial, endotheliochorial, and hemochorial (Figure 3). The 'syndesmochorial' classification was later replaced with 'synepitheliochorial' after it was demonstrated to be a more accurate description of this placental type present in ruminant species (Wooding 1982a; 1982b; 1992).

(1) Epitheliochorial - The epitheliochorial placental type is the least invasive form of placenta. There is generally little to no invasion of the endometrium, resulting in the uterine LE being in direct apposition to the fetal chorion (Figure 3A). Species with this type of placenta are able to reduce the distance required for physiological exchange by interdigitation of vascularized chorionic and uterine luminal microvilli. This allows the fetal and maternal vasculatures to lie in close proximity to each other, which serves to decrease diffusion distance (Enders and Blankenship 1999; Wooding and Burton 2008; Furukawa, Kuroda, and Sugiyama 2014). There are a total of 6 tissue layers separating fetal and 
maternal blood: fetal endothelium, fetal connective tissue, fetal TE, uterine LE, uterine connective tissue, and maternal endothelium.

Epitheliochorial placentation is found in many species of the Artiodactyla (e.g. pigs, whales, camels) and Perissodactyla (e.g. horses, rhinos) orders. This form is also represented in prosimian primates and Pangolins (Philidota order). Even within this category, there are distinct adaptations. The horse serves as a good example. In the horse, there is transient invasion of the LE and subepithelial stroma by a subset of trophoblasts known as chorionic girdle cells. These cells aggregate and differentiate into structures known as endometrial cups. The uterine LE is briefly lost during the migration of these cell but it is quickly recovered. Later in pregnancy the endometrial cups are destroyed by the maternal immune system. Invasion of the endometrium by girdle cells starts around day 36 of pregnancy; endometrial cups are fully formed around day 70 and their destruction begins around day 100. Overall, the horse exhibits a modified epitheliochorial placenta for only about $1 / 4$ of the pregnancy (Allen, Hamilton, and Moor 1973; Hamilton, Allen, and Moor 1973; A. C. Enders and Liu 1991; Carter and Enders 2004; P. Wooding and Burton 2008)

(2) Synepitheliochorial - Ruminants within the Artiodactyla order are known to have a modified type of epitheliochorial placenta known as 'synepitheliochorial'. The 'syn-' prefix is derived from the distinguishing feature of this placenta: a fetal-maternal syncytium. This type of placenta was originally named 'syndesmochorial' because it was believed that the uterine LE was lost and the chorion was in direct contact with uterine connective tissue (Grosser 1909; 1927). Later work from Wooding and colleagues (F. B. Wooding 1982a; 1982b; 1984; F. B. P. Wooding 1992) demonstrated that the LE is not lost, rather, it is modified. In ruminant placentation, giant BNCs migrate across the trophoblast-LE interface and fuse with uterine luminal epithelial cells to form a unique trinucleated fetal-maternal hybrid cells. Depending on the species and/or the developmental stage, continued fusion of BNCs can lead to the formation of large multinucleated syncytial plaques. Only the caruncular uterine epithelium 
is modified in this way, however (Figure 3B). The LE within the intercaruncular areas remains intact and the fetal-maternal interface in these areas resemble an epitheliochorial arrangement (Wooding 1982b; 1984; Wooding et al. 1996). Overall, the number of tissue layers separating fetal and maternal blood is the same as for epitheliochorial placentation.

In sheep and goats, the syncytial plaques can persist throughout the entire pregnancy. In cattle, however, syncytial formation is transient. By day 40 of pregnancy most, if not all, of the syncytial plaques have been overgrown by the residual rapidly dividing luminal epithelial cells. Throughout the rest of the pregnancy, the placenta resembles an epitheliochorial placenta. BNC continue to migrate across the interface and form trinucleated cells, however, these cells are short-lived and are soon phagocytosed back into the TE layer (Wooding 1992).

(3) Endotheliochorial - An endotheliochorial type of placentation is more invasive than the previously mentioned placental types. This type of placentation involves the breaching of both the uterine $L E$ and its underlying connective tissue so that the chorion can be in direct apposition to the maternal endothelial basement membrane (Figure 3C). In this type of placenta the fetal and maternal capillaries are usually separated by 4 tissue layers: fetal endothelium, fetal connective tissue, fetal TE, and maternal endothelium. This type of placenta has be studied most extensively in carnivores, although it is widely represented in eutherians (Enders and Carter 2006; Wooding and Burton 2008; Furukawa, Kuroda, and Sugiyama 2014)

(4) Hemochorial - Hemochorial placentation is the most invasive type of placentation. In this form, the invasive trophoblasts can erode or circumvent the uterine LE, connective tissue, and the endothelium of the maternal vasculature to establish direct contact with maternal blood. This leaves only 3 tissue layers separating fetal and maternal blood: fetal endothelium, fetal connective tissue, and fetal TE (Figure 3D). Hemochorial placentation can be further subdivided to described how many layers 
of trophoblasts lie between the maternal blood and fetal connective tissue. When the placenta only contains a single layer of trophoblasts between maternal blood and fetal connective tissue it is called 'hemomonochorial'. When the placenta contains 2 layers of trophoblasts (usually a layer of syncytiotrophoblasts and a layer of cytotrophoblasts) it is called 'hemodichorial'. When the placenta contains 3 layers of trophoblasts it is called 'hemotrichorial' (Enders 1965; Enders and Blankenship 1999; Wooding and Burton 2008). This type of placentation is found in rodents, lagomorphs and higher primates.

\section{Figure 2}


Figure 3 


\section{Additional placental classifications}

There are additional ways to characterize a placenta that provide further insight into how physiological exchange is mediated. One way is by classifying the type of fetal-maternal interdigitation. The tissue at the fetal-maternal interface can be classified into 5 conformational groups: folded, lamellar, trabecular, villous, and labyrinthine (Figure 4). The simplest of these forms is the 'folded' type, an arrangement seen in swine placentation (Figure 4A). This type of interdigitation involves apposition of the folded surface of the TE to the primary and secondary folds of the uterine LE (G. J. King 1993). When these folds are further drawn out into tall, closely packed sheets, the result is a 'lamellar' conformation (Figure 4B). This forms an elaborate interdigitation of elongated, parallel chorionic and uterine lamellae that is observed in the placenta of carnivores (Leiser and Koob 1993). A 'trabecular' interface contains incomplete folds that engage in secondary branching (Figure 4C; B. F. King 1993). This conformation is observed in some primates. A 'villous' type interface consists of complex chorionic branching that results in a three-dimensional tree-like structure (Figure 4D). This type of configuration is observed in human placentation (Wooding and Flint 1994). Finally, the most complex interface is a 'labyrinthine' configuration. It is comprised of a complex, three-dimensional, interconnected meshwork of chorionic villi which creates cavities that can fill with maternal blood for placental exchange (Figure 4E; Pijnenborg et al. 1981). This type of interface is seen in the placenta of rodents.

Placentae can also be characterized by the loss of maternal tissue at parturition. A 'deciduate' type of placentation results in a variable loss of maternal tissue with the fetal membranes at parturition. This generally correlates with placentas that involve erosion of maternal tissue (e.g. endotheliochorial and hemochorial). A 'non-deciduate' type of placenta separates at the fetal-maternal interface at parturition with very little to no loss of maternal tissue. 
Finally, placentae can be classified by specialized accessory placental structures. Hemophagous zones are one such specialized structure that is defined as an 'area where the chorion and maternal tissue are widely separated by stagnant, but not necessarily clotted, maternal blood' (Wooding and Burton 2008). Trophoblasts in hemophagous zones are usually large and full of vacuoles containing phagocytosed erythrocytes. It is hypothesized that digestion of hemoglobin in these zones provides a source of fetal iron (Baker and Morgan 1973). Areolae are another type of specialized placental structure that are similar to hemophagous zones except, rather than filling with maternal blood, they fill with glandular secretions for endometrial glands. Areolae, most commonly found in epitheliochorial type placentation, consist of areas of columnar trophoblasts overlying the mouths of endometrial glands (A. C. Enders and Carter 2006; P. Wooding and Burton 2008). The areolar trophoblasts phagocytose the uterine glandular secretions known as 'histotroph' to supply the developing placenta with nutrients such as iron, amino acids, carbohydrates, proteins, and lipids (A. C. Enders and Carter 2006; Thomas E. Spencer 2014). 
Figure 4 


\section{Binucleate cells}

Ruminant ungulates possess a cotyledonary synepitheliochorial placenta. This form appears to be an adaptation of the epitheliochorial placenta (Roberts, Green, and Schulz 2016). As previously described, a large area of this placenta has retained an epitheliochorial phenotype, consisting of areas of simple apposition of the fetal and maternal tissues without loss of any maternal tissue. However, the 'syn' designation is derived from the multinucleated syncytium that is formed within specialized structures known as placentomes (the combined structure consisting of a maternal caruncle and a fetal cotyledon). The syncytium in ruminant ungulates is derived from the fusion of migrating fetal BNC with maternal endometrial epithelial cells (Wooding 1984; and 1992). The BNCs are very important for the structure and function of the ruminant placenta. In the following section, this review will attempt to describe the origins and the functional and structural significance of the BNC in the ruminant placenta.

\section{Binucleate cell origin, structure, and differentiation}

BNCs first appear in the ruminant placenta around the start of conceptus attachment. In cattle and goats this is around day 18 post coitus (Greenstein, Murray, and Foley 1958; Wango, Wooding, and Heap 1990) and in sheep this is around day 16 post coitus (Boshier 1969). Eventually, BNCs make up 15$20 \%$ of the TE of the mature ruminant placenta (Wooding 1992). Of the BNC population, $15-20 \%$ of those are migrating across the microvillar junction at any given time (Wooding 1983; Wooding et al. 1986).The origins of BNCs are unclear. There are no indications of stem cells or reserves in the TE that give rise to giant BNCs. However, the common assumption is that they originate from the columnar mononucleate cells (Wooding 1992; Wooding et al. 1994). Indeed, any mononucleate trophoblast appears to be capable of producing a BNC. It has been proposed that BNC are generated first through asymmetrical mitoses of mononucleate trophoblasts that gives rise to two distinct cells: a normal mononucleate trophoblast that incorporates into the TE and an irregular mononucleate cell that no 
longer rests on the basement membrane or participates in apical tight junctions. This cell would then undergo acytokinetic mitosis, resulting in a binucleate cell with two diploid nuclei (Wooding and Flint 1994; Klisch et al. 1999). Klisch and colleagues were able to visualize irregular mitoses in 'free floating' trophoblasts that did not result in cytokinesis, thereby supporting the previous theory.

There is also little known about what drives syncytial formation during synepitheliochorial placentation. Research on this area suggests that endogenous retroviruses may be involved in placental growth, BNC differentiation, and BNC fusion (Dunlap et al. 2005 and 2006). In sheep, the endogenous beta retrovirus Jaagsiekte sheep retrovirus (enJSRV) envelope (env) RNAs have shown to be expressed throughout gestation in the endometrium and in the conceptus. However, when enJSRV env was knocked down in the endometrium and conceptus, conceptus growth was notably retarded by day 16 and there were a lack of BNC in the TE (Dunlap et al. 2005). This would suggest that this endogenous retrovirus plays a major role in conceptus growth and elongation as well as BNC formation. Bovine trophoblasts also express endogenous retroviral RNAs, most notably is bovine endogenous retrovirus envelope element-like transcript-A (Berve-A), which is similar to human syncytin-1 (Koshi et al. 2011 and 2012). Berve-A as well as Berve-K1 env are detected in bovine trophoblasts during the peri-implantation period and their expression is enhanced during BNC induction. Like enJSRV in the sheep, these endogenous retroviruses may be involved in the formation of BNC ( Koshi et al. 2012).

Once a giant BNC is formed, it is considered terminally differentiated and has lost its ability to proliferate. However, there is still a maturation process that must occur. The maturing BNC not only increases in size but also develops an extensive network of rough endoplasmic reticulum and a large Golgi apparatus ( Wooding 1992). The mature BNC produces a large amount of proteins packaged into cytoplasmic granules; not unexpectedly, they possess large endoplasmic reticulum and Golgi. The cytoplasmic granules can occupy more than $50 \%$ of the total cell volume of a mature BNC. Contained within these granules is a mixture of proteins and glycoproteins; most notable they contain placental 
lactogen and pregnancy-associated glycoproteins (PAGs) (Wooding 1982a; Duello, Byatt, and Bremel 1986; Green et al. 2000; Nakano et al. 2001).

Interestingly, the contents of the BNC granules may differ at different times throughout pregnancy; the granule contents can also differ with the location of the BNC in the placenta. This led to the hypothesis that, while all BNC ultrastructure is very uniform, there may exist subpopulations of BNCs that contain different proteins within their cytoplasmic granules (Wooding 1992). Immunostaining of bovine placentae with the BNC-specific monoclonal antibody SBU-3 revealed that staining did not occur until day 30 of pregnancy, even though BNC are present in the TE by days 18-20 (Morgan et al. 1989). In the ovine placenta, cytoplasmic granules of BNC within the placentome have been shown to contain both placental lactogen and SBU-3 antigen, however, neither of these were present in the interplacentomal BNC (Lee, Wooding, and Brandon 1986a, b, c; Lee, Gogolin-Ewens, and Brandon 1986). Furthermore, spatial differences in potential BNC subpopulations have been noted in deer. A higher concentration of SBU-3 positive BNC were present in the chorionic villous tips compared to the base of the villi (Lee, Gogolin-Ewens, and Brandon 1986). The SBU-3 antigen(s) are probably likely PAGs and more than likely a specific sub-group of PAGs (Atkinson et al. 1993). If that is the case, then the staining patterns seen with this antibody may suggest that different BNC produce different PAGs based on stage of pregnancy and location within the placenta.

\section{Binucleate cell function: Migration and cell fusion}

BNCs have two characteristic functions; 1) They migrate across the microvillar junction from the TE into endometrial epithelium, fuse with an endometrial epithelial cell to form a trinucleate cell, and eventually form a syncytium that is either transient (cattle) or persistent throughout pregnancy (sheep).

2) BNCs synthesize and deliver their secretory products into the uterine stroma after fusion with an endometrial epithelial cell (Wooding 1992). 
After conceptus attachment, at any time during pregnancy BNCs can be seen migrating across the microvillar junction. About $20 \%$ of the total BNC population are actively migrating at any given time (Wooding 1983). In sheep and cattle, the earliest occurrence of BNC migration has been recorded as day 18 and 20, respectively. The free BNCs are not anchored to the TE by the basement membrane. Pseudopodia on the BNCs form that push through the TE apical tight junctions and make contact with the apical surface of an endometrial epithelial cell. The contact surface made between the BNC pseudopodium and the epithelial cell appears flat and without microvilli. The cytoplasm in the pseudopodium is devoid of organelles but abundant in small vesicles. This creates the initial fusion surface for the formation of a trinucleate cell (Wooding et al. 1994; Klisch et al. 1999). As the cytoplasm of the two cells mix during fusion, the cytoplasmic granules of the BNC move towards the maternal size of trinucleate cell. The secretory granules are shortly after exocytosed from the trinucleated cell towards the endometrial stroma where the contents either accumulate or then diffuse into the maternal circulation (Wooding et al. 1994).

This process is continuously occurring throughout pregnancy and its continuation is what ultimately forms the fetal-maternal hybrid syncytium that is characteristic of synepitheliochorial placentae. In sheep the syncytium can be quite extensive, consisting of syncytial 'plaques' with 20-24 nuclei. The syncytium in sheep also persists throughout pregnancy, whereas in cattle it is transient and usually disappears by day 40 (Wooding 1992). After day 40 of pregnancy in cattle, migration and fusion of BNC is still occurring, however, the trinucleate cells that are formed are short-lived and are quickly phagocytosed back into the TE (Wooding and Wathes 1980). Whether the syncytium is persistent or short-lived, continued migration and fusion of the BNC contributes to the development and growth of the placenta throughout pregnancy. 


\section{Products of binucleate cells}

Trophoblastic BNC are known to produce many proteins and glycoproteins. With their large extensive networks of endoplasmic reticulum and large Golgi they can make large quantities of proteins and package them into secretory granules (Wooding 1992). One such protein is placental lactogen (PL), also known as chorionic somatomammotropin. PL is a placental polypeptide hormone with a molecular weight of 30-32 kDa (Murthy et al. 1982). It can be measured in both the maternal and fetal circulation of cattle, sheep, and goats during pregnancy and is immunolocalized to the BNC. More specifically, it is localized in the cytoplasmic granules of the BNC (Duello, Byatt, and Bremel 1986). PL hormones (there are at least 2 identified in cattle) exhibit both lactogenic and somatogenic properties in the mother and the fetus (Alvarez-Oxiley et al. 2008). Binding sites for PL are present in the fetal tissues, especially the fetal liver, which has given rise to the belief that PL may act as a fetal growth hormone (Thordarson et al. 1987).

BNCs also produce some steroid hormones. Progesterone is essential for pregnancy establishment and also for maintaining pregnancy. Cattle primarily rely on the CL as the main source of progesterone throughout pregnancy. However, sheep depend on placental progesterone for the last two-thirds of the pregnancy. Placental progesterone primarily comes from trophoblasts, both mononucleate and binucleate. Perhaps because of their size, BNC produce significantly more progesterone per cell than mononucleate trophoblasts. Enriched suspensions of bovine BNC produced large amounts of progesterone over a 4 hour period. They also produced $\mathrm{PGI}_{2}$ and $\mathrm{PGE}_{2}$. Both $\mathrm{PGI}_{2}$ and $\mathrm{PGE}_{2}$ are important in maintenance of the $\mathrm{CL}$ during pregnancy (Reimers, Ullmann, and Hansel 1985). Sheep appear to rely more on placental progesterone and probably benefit from the high production capacity of the BNC. Cattle probably benefit more from the BNC prostaglandins because the $\mathrm{CL}$ continues to be the main source of progesterone during pregnancy. 
Lastly, the major products of the BNCs are the PAGs. A more detailed descriptions of these BNC products is discussed in the next section of this review. Briefly, the PAGs are a large family of placental aspartic proteinases produced by both the mononucleated and BNC trophoblasts of the ruminant and porcine placenta (Butler et al. 1982; Xie et al. 1991; Zoli et al. 1991). Ruminant PAGs can be put into two main categories: ancient and modern. The modern PAGs are exclusively produced by BNC (Figure 5B). Ancient PAGs can be produced by either the mononucleate trophoblasts or BNC or both (Figure 5A, 5C, 5D; Xie et al. 1991; Green et al. 2000). Like PL, some PAGs can enter the maternal circulation during pregnancy because of the migratory and fusogenic properties of the BNC. The PAGs in the BNC granules that get exocytosed into the uterine stroma either accumulate in the uterine stroma or diffuse into nearby endometrial capillaries (Green et al. 2000 and 2005; Wooding, Roberts, and Green 2005). Accumulation of PAGs in the maternal circulation forms the basis for most modern pregnancy tests for cattle and sheep (Green et al. 2005a). There is evidence to suggest that there may be subpopulations of BNCs that produce different PAGs based on the stage of pregnancy and location in the placenta (Lee, Wooding, and Brandon 1986a; 1986c; Lee, Gogolin-Ewens, and Brandon 1986; Wooding 1992). Currently, the function(s) of the PAGs are unknown. There is evidence to suggest that the PAGs may play a role in matrix remodeling at the interface and/or in the endometrium (R. M. Wallace et al. 2019), they may serve as luteotrophic agents during pregnancy (Weems et al. 2007), or they may be serving immunomodulatory roles (Hoeben et al. 1999). The next section of this review will attempt to give a more detailed overview on this complex family of placental glycoproteins with highlights on the gene family, its expression patterns, and proposed functions. 
Figure 5 


\section{Bovine Pregnancy-Associated Glycoproteins}

\section{Overview}

The pregnancy-associated glycoproteins (PAGs) are products of a gene family of placental aspartic proteases that are present in members of the Cetartiodactyla order (even-toed ungulates and cetaceans; Green and Hennessy 2018). They are produced exclusively by the trophoblasts of the placenta (mononucleated and BNC) throughout pregnancy. They were originally identified by three independent research groups from purified bovine placental extracts (Butler et al. 1982; Sasser et al. 1986; A P Zoli et al. 1991; S. C. Xie et al. 1991; Mialon et al. 1994). The protein that was initially isolated was named pregnancy-specific protein B (PSPB) and pregnancy-specific protein of sixty thousand molecular weight (PSP60). Today we know this protein as the first member of the bovine PAG family, PAG1 (Guruprasad et al. 1996). Since then, the characterization of this gene family has revealed an unanticipated complexity in the number and placental expression of the PAGs. At least two dozen distinct PAGs have been identified in cattle and sheep. Other species within Bovidae have comparable numbers of PAG genes; while there are fewer in pigs (Suidae) and whales (Cetacea). Although we still don't know much about the roles of the PAGs, they likely play important roles in placental function and pregnancy in even-toed ungulates. This review of the bovine PAGs will attempt to provide the reader an overview of the gene family, its products, and the potential roles they may play in pregnancy.

\section{The PAG family}

The PAGs are believed to have arisen as the result of a duplication and subsequent expansion of an ancestral pepsin F-like gene around the time that the Cetartiodactyla order arose (Hughes et al. 2000). This pepsin F-like gene itself is represented in most mammals and it is thought to have arisen from a duplication of pepsin $A$. Pepsin $F$ and pepsin $A$, are members of an enzyme family known as aspartic proteinases. Phylogenetic analyses of the PAGs shows them clustering with other known 
aspartic proteinases such as the cathepsin D, cathepsin E and renin (Hughes et al. 2003; Green and Hennessy 2018). Phylogenetic analyses also show a segregation of the ruminant PAGs into two distinct groups termed 'ancient' and modern' based on the times at which they arose during Artiodactyl evolution (Hughes et al. 2000). The ancient PAGs are predicted to have emerged approximately 87 million years ago and consist of bovine PAG (boPAG) $-2,-8,10,-11$, and -12 (Hughes et al. 2000). The modern PAGs are by far the most numerous and make up the rest of the PAG family. They are predicted to have arisen approximately 52 million years ago (Hughes et al. 2000) and have only been observed in the Ruminantia (B. P. V. Telugu, Walker, and Green 2009). The Bovidae (cattle, sheep, etc.), in particular, have experienced a large expansion of the PAGs with at least two dozen confirmed PAG genes plus numerous pseudogenes, adding to the complexity of this gene family.

The PAGs belong to the aspartic proteinase group of enzymes and they share the common structural features of these enzymes. They consist of a symmetrical bi-lobed, bean shaped structure with a cleft in between the lobes that forms the peptide binding/catalytic domain. Within the binding cleft, each lobe contributes a catalytic aspartic acid residue within a conserved sequence motif (Asp-Thr-Gly-Ser/Thr-Ser/Thr) that is preceded by two hydrophobic amino acid residues (Dunn 2002; Green and Hennessy 2018). The catalytic aspartic acid residues give rise to the name of this group of enzymes: 'aspartic' proteinases (Davies 1990). The catalytic aspartic acids participate in the hydrolysis of peptide bonds by orientating and stabilizing a water molecule between them. This water molecule can then serves as a nucleophile during hydrolysis of the bound peptide (Dunn 2002; Green and Hennessy 2018). The majority of the PAGs contain these conserved aspartic acid motifs, along with other 
conserved regions found in this enzyme class. Consequently, most have the potential to function as active aspartic proteinases. This activity has been demonstrated in some PAGs such as boPAG2, boPAG12 and porcine PAG2 (Telugu and Green 2008; Telugu et al. 2010) However, there are a few notable exceptions. For example, the first of the bovine PAGs to be cloned, boPAG1, has the catalytic aspartic acid residues but one of the invariant glycine residues in the $\mathrm{N}$-terminal catalytic domain is replaced with an alanine (Xie et al. 1991). BoPAG10 also has an amino acid substitution in its C-terminal catalytic domain. Further cloning of the PAGs has revealed multiple bovine and ovine PAGs with mutations within the conserved catalytic domains that may render these proteins enzymatically inactive (Guruprasad et al. 1996; Sancai Xie et al. 1997; Green et al. 2000). At present, it is unclear if the enzymatic activity is relevant to their biological role(s) in vivo.

\section{Temporal and spatial expression of PAGs}

\section{Expression at the interface}

The complexity of the PAG family not only comes from the large number of family members but also in their expression patterns throughout pregnancy. Because of the large expansion of the PAGs in Ruminantia, the expression patterns of various PAGs have been well studied throughout pregnancy. Initially the characterization of PAG expression was done using in situ hybridization in placentomes of cattle, sheep, and goats (Garbayo et al. 2000; Green et al. 2000). These studies revealed differences in cell-specific expression patterns of the PAGs. The majority of the PAGs studied were expressed solely by the BNCs. However, a few of the PAGs were expressed by all trophoblasts (mononucleated and binucleated). The cell-type expression seemed to correspond with the 'ancient' versus 'modern' divisions of ruminant PAG phylogeny;. In general, the modern PAGs are BNC-specific. Both Mrna and 
protein expression of these PAGs are restricted to the BNC (Green et al. 2000). The ancient PAGs are a bit more complicated because they can be expressed by the mononucleated trophoblasts, BNC, or both. For example, boPAG1 is a modern PAG that is expressed solely by the BNCs (Xie et al. 1991). BoPAG2 is an ancient PAG that is expressed uniformly by all trophoblasts (Sancai Xie et al. 1994). Immunolocalization of boPAG2 agrees with the in situ localization (R. M. Wallace et al. 2015), however, one immunolocalization study did not observe boPAG2 staining in the BNC (Touzard et al. 2013). BoPAG8 is also an ancient PAG but it expressed only by the mononucleated trophoblasts (R. M. Wallace et al. 2015). In contrast, with in situ hybridization boPAG10 (an ancient PAG) appears to have a broad trophoblastic distribution (Green et al. 2000) but immunolocalization localizes this PAG specifically to BNCs (R. M. Wallace et al. 2015). These expression patterns do not apply to all species, however. There are exceptions, such as deer PAG3 (a modern PAG) that is expressed in both mononucleated trophoblasts and BNC (Brandt et al. 2007).

With the exception of the Tragulidae family (mouse deer), which have a diffuse placenta (Kimura et al. 2004), Ruminantia members have developed a unique strategy for utero-placental exchange. As discussed previously in this review, the ruminant placenta consists of two distinct areas of exchange; 1 ) the intercotyledonary regions where the exchange surface is that of an epitheliochorial placenta, and 2) the cotyledonary regions where the exchange surface is increased by networks of cotyledonary and caruncular villi and syncytial cells or multinucleated syncytial plaques. While BNCs are present in both of these regions, only in the placentome do they seem to cross the interface into the endometrial epithelium (Wooding, Morgan, and Adam 1997). This provides a new delivery mechanism for the BNC-produced PAGs that is distinct from the intercotyledonary regions of the placenta. The population of PAGs expressed between these two regions often differs as well as the accumulation of PAGs at the interface or within the tissues. BoPAGs1, $-2,3,-15,-17$, and -21 expression is greater in the cotyledons than in the intercotyledonary TE (Touzard et al. 2013). Even within the cotyledons there may 
be uneven spatial distribution of the PAGs. Immunolocalization of boPAG2 revealed that this protein is not equally distributed within the placentome. BoPAG2-positive trophoblasts were seen primarily in the secondary and tertiary cotyledonary villi and little to no staining in the villous stems or the villous base (Touzard et al. 2013). In contrast, boPAG8, -10, -11, and -12 expression is greater in the intercotyledonary TE throughout pregnancy. Western blots of boPAG11 (an ancient BNC-specific PAG) extracted from cotyledonary and intercotyledonary TE agrees with the Mrna expression, however, immunostaining labeled boPAG11-positive BNCs in both tissues. The intercotyledonary TE showed an even distribution of boPAG11 but in the cotyledons, boPAG11 BNCs were restricted to the chorionic plate and were absent in the secondary and tertiary villi (Touzard et al. 2013). Some PAGs are capable of accumulating in the uterine stroma. As discussed previously in the binucleate cell section of this review, BNCs within the placentome can migrate across the utero-placental interface to enter the uterine LE and fuse with an epithelial cell. Fusion of the BNC with a LE cell allows the BNC granules to be exocytosed into the endometrial stroma (Wooding 1992). It's very likely that the PAGs produced by the BNCs within the placentome can enter and accumulate in the uterine stroma as a result. Wooding et al. were the first to report a systematic evaluation of the boPAGs by immunolocalization (Wooding, Roberts, and Green 2005). They reported that immunostaining of some boPAGs, primarily modern PAGs, also stained the endometrial connective tissue of the caruncular villi as early as day 30 of pregnancy. This pattern of villous connective tissue staining was seen throughout the rest of pregnancy only for the modern PAGs and within the placentome. In the intercotyledonary TE, immunostaining for modern PAGs was restricted to BNCs and no staining of the endometrium was noted (Wooding, Roberts, and Green 2005). Because the ancient PAGs are often produced by mononucleated trophoblasts that do not migrate across the interface, no uterine connective tissue staining was ever observed. However, accumulation of these PAGs was observed along the microvillar junction as early as day 20 or pregnancy. This pattern is similar to what is seen in pigs, where fetal-maternal interdigitation is less extensive and 
there is no BNC migration. Accumulation of these PAGs along the microvillar junction was observed throughout pregnancy (Wooding, Roberts, and Green 2005). It is clear that each PAG may possesses a unique spatial expression pattern within the placenta. Whether or not these expression patterns contribute to their functions is still unclear.

While the PAGs are not distributed evenly throughout the placenta at a given time, the same is true of their temporal expression throughout pregnancy. Early efforts to characterize the expression of the PAGs throughout pregnancy were done by Green et al. using Rnase protection assays to identify various PAG transcripts from day 25 of pregnancy to term. The authors demonstrated that each PAG seems to have a distinct temporal expression pattern. Some PAGs are expressed throughout pregnancy while expression of other may not turn on until a mature chorioallantoic placenta has formed. One commonality, however, is that expression of the BNC-specific PAGs studied was invariably absent in term placenta (Green et al. 2000). Patel and colleagues focused on boPAG1 and boPAG9 (modern PAGs) expression from days 30-250 of pregnancy. They showed that expression of boPAG9 is up to 10 times greater in early pregnancy compared to boPAG1 expression. BoPAG1 Mrna abundance seemed to increase as pregnancy progressed, peaking in cotyledonary tissue on day 250 (Osman V. Patel et al. 2004). These results were consistent with the earlier study by Green et al. that showed that the earliest expression of boPAG1 was seen on day 45 while boPAG9 was highly expressed on day 25 (Green et al. 2000). Work by Telugu et al. focused on temporal expression of the ancient PAGs. They demonstrated that each of the ancient PAGs has a distinct temporal expression pattern that, with the exception of boPAG10, the ancient PAGs are most abundant in early pregnancy. BoPAG10 displays an opposite expression pattern that is low in early pregnancy and peaks at term (Telugu, Walker, and Green 2009).

Overall, the PAGs express a range of expression patterns, both spatial and temporal, throughout pregnancy. In general, the ancient PAGs are usually expressed early in pregnancy at a greater abundance than the modern PAGs (Patel et al. 2004; Telugu, Walker, and Green 2009; Touzard et al. 
2013). The modern PAGs vary considerably in their temporal expression but most of them appear to be absent or minimally expressed in term placenta (Green et al. 2000; Touzard et al. 2013). The cellspecific expression combined with the distinct spatial and temporal expression patterns of the PAGs sheds light on the complexity of this family of aspartic proteinases. It is reasonable to assume that the unique expression patterns of the PAGs is reflective of their functional role(s) during pregnancy. What those roles are remains unclear but several possibilities are discussed in the 'Possible functions' section of this review below.

\section{Use of PAGs in pregnancy diagnosis}

Pregnancy detection in cattle traditionally was done via rectal palpation. While this is still performed today, farmers and researchers recognize that this is not the most reliable method of pregnancy diagnosis, particularly when performed in early pregnancy. In the '70's the measurement of progesterone concentration in the milk became a popular tool and was found to be relatively accurate and this approach became a useful method for researchers and producers (Pennington, Spahr, and Lodge 1976; Pennington, Schultz, and Hoffman 1985; Laing and Heap 1971; Heap et al. 1976). However, there are a lot of factors that affect progesterone concentrations that often led to incorrect pregnancy diagnosis; these include 1) variations in the length of the estrous cycle (short vs. long cycle) and 2) embryonic mortality (Pennington, Schultz, and Hoffman 1985). In other species, assays for placental proteins, such as human chorionic gonadotrophin $(\mathrm{Hcg})$ in humans, was commonly used to diagnose pregnancy. When the PAGs were first discovered (originally called PSPB) they became a prime candidate for pregnancy diagnosis. As discussed previously in the binucleate cells section of this review, the BNC-produced PAGs are released into the endometrial stroma after fusion of BNCs with LE cells. The PAGs released into the uterine stroma often accumulate around capillaries in the shallow stroma which allows them to enter the maternal circulation, making them a good candidate for placentalspecific pregnancy diagnosis (Wooding, Roberts, and Green 2005). Sasser et al. were the first to develop 
a radioimmunoassay targeting PSPB in the maternal serum of cows. They were able to reliably detect PSPB as early as day 30 of pregnancy and noticed that PSPB serum concentrations rose through pregnancy and peaked about two days before parturition (Sasser et al. 1986). Later studies were able to develop an enzyme-linked immunosorbent assay (ELISA) for the detection of PAGs in bovine and ovine sera (Green et al. 2005a; Egen et al. 2009). These studies were able to create a PAG serum profile that mirrored the profile recorded by Sasser et al. The bovine ELISA uses monoclonal anti-boPAG antibodies that detect a rise in serum PAG concentrations around days 23 of pregnancy and could diagnose pregnancy by day 28 with 93-96\% accuracy (Silva et al. 2007; Pohler et al. 2013). In cattle, serum PAG concentration begins to increase to from day 23 to about day 36, reaching an average concentration of $8.75 \mathrm{ng} / \mathrm{Ml}$ by day 28 and $12.3 \mathrm{ng} / \mathrm{Ml}$ by day 35. Between days 36 and 60 PAG concentrations decline to about half of that on day 35, but then start to steadily increase through the remainder of pregnancy. During the last week of pregnancy, serum PAG concentrations rise dramatically and peak between $500 \mathrm{ng} / \mathrm{Ml}$ to as much as $5 \mathrm{ug} / \mathrm{ml}$, depending on the assay that is employed. Concentrations decline after parturition, but persistent circulating PAGs post-partum can limit this method of pregnancy detection for a subsequent pregnancy. PAGs in the postpartum circulation creates a potential for false positives if an animal were to be rebred too soon after calving. The ELISA established by Green et al. attempted to address this scenario by using monoclonal antibodies that target PAGs that were present early, were less abundant at term, and exhibited a shorter half-life (Green et al. 2005a). Overall, this ELISA has become a useful tool for ruminant pregnancy diagnosis. Today various forms of this assay have been developed commercially and are regularly used by companies to provide pregnancy diagnostic services.

In addition to pregnancy diagnosis, PAGs circulating in maternal blood have also been used to characterize fetal number, fetal health, parity, lactation status, and early embryonic loss $(\mathrm{O}$. V. Patel et al. 1995; J. M. Wallace et al. 1997; Chavatte-Palmer et al. 2006; López-Gatius et al. 2007; Constant et al. 2011; Pohler et al. 2013). Additionally species differences are indicated between genotypes (Lobago et 
al. 2009; Mercadante et al. 2013). For example, circulating PAGs in cattle with Bos indicus genetics are higher than cattle with Bos taurus genetics at a mean gestational age of 53 days (Mercadante et al. 2013). Placental insufficiencies, such as those from pregnancies of somatic clones, are also known to cause elevated systemic PAG concentration. Somatic clone pregnancies have been known to present with higher circulating PAG concentrations from days 24-50 (Chavatte-Palmer et al. 2006) and day 62 of pregnancy (Constant et al. 2011).

\section{Possible functions of the PAGs}

\section{Matrix remodeling and adhesion}

Though the PAG family has been extensively studied over the last forty years, little is known about the role(s) that the PAGs play during pregnancy. Speculation have been made based on their localization patterns during pregnancy and their predicted proteolytic activity, if any. Some of the PAGs are known to be proteolytically active. BoPAG2, boPAG12, and porcine PAG (poPAG)-2 have all been shown to cleave a common aspartic proteinase FRET substrate (Telugu and Green 2008; Telugu, Walker, and Green 2009). These PAGs have been shown to accumulate along the microvillar junction, so it is reasonable to predict that some PAGs could be acting to proteolytically process proteins at this location. Latent growth factors at the interface are potential substrates for aspartic proteases such as PAGs (Munger et al. 1998; Rifkin et al. 1999; Moussad et al. 2002). If PAGs at the interface are proteolytically active, then it's likely that PAGs accumulating in the uterine stroma could be proteolytically active as well. It's also possible that the PAGs could affect matrix remodeling by upregulating expression of MMPs. In bovine endometrial explants, treatment with PAGs (a mixture of modern PAGs) increased the endometrial expression of several MMPs (R. M. Wallace et al. 2019). Whether this is a direct result of PAG action or a downstream effect of the PAGs is unclear. It is also not known whether this same response occurs in vivo. 
Another possible role for the PAGs is as adhesion proteins. All PAGs contain an amino acid binding cleft and several glycosylation sites on the protein's surface. The binding cleft could allow for interactions with other proteins, especially proteins at the microvillar junction, such as integrins. The carbohydrates could conceivably interact with lectins, thereby permitting the PAGs to act as bifunctional linking molecules (Klisch et al. 2007; Wooding, Roberts, and Green 2005). So long as the Ph at the microvillar junction is not too low, the enzymatically active PAGs could also serve as binding/linking molecules (aspartic proteinase activity is typically greatest at low $\mathrm{Ph}$ ). If the $\mathrm{Ph}$ were to drop and the PAGs were to become activated, which might occur around parturition, then the active PAGs could begin to cleave their bound proteins and sever the protein-protein connections between the placenta and endometrium (Green and Hennessy 2018). Very little research has been done on this potential aspect of the PAGs so this is all speculative.

\section{Luteotrophic}

Many PAGs are first expressed by the placenta during conceptus attachment, the time when most early embryonic loss occurs (Short 1969; Martal et al. 1979; Godkin et al. 1984). Therefore, it is not unreasonable to predict that the PAGs may play a role in early pregnancy maintenance. Early studies demonstrated that PAG/PSPB could increase production of $\mathrm{PGE}_{2}$ (Del Vecchio, Sutherland, and Sasser 1996), which in turn could increase progesterone production by bovine luteal cells in vitro (Del Vecchio, Sutherland, and Sasser 1995). Later studies by Weems et al. showed that PAGs increased the production of $\mathrm{PGE}_{2}$ relative to $\mathrm{PGF}_{2 \mathrm{a}}$ in cultured bovine luteal and endometrial cells and explants (Weems et al. 1998; 2003; 2007). This is relevant because $\mathrm{PGE}_{2}$ is considered to be a luteotrophic prostaglandin (Shelton et al. 1990; Arosh et al. 2004) and its concentration in relation to PGF $2 a$ is important in determining the fate of the CL (Christenson et al. 1994; Ziecik 2002; Kaneko and Kawakami 2009). Therefore, it is has been predicted that the PAGs may serve a luteotrophic role during early pregnancy by regulating $\mathrm{PGE}_{2}$ and $\mathrm{PGF}_{2 a}$ production by the $\mathrm{CL}$ and/or the endometrium. 


\section{Immunomodulatory}

The PAGs have also been implicated in modulation of the maternal immune system in cattle. Circumstantial evidence was recorded by Dosogne et al. who noticed that peak plasma PAG concentrations at parturition immediately preceded a decrease in circulating polymorphonuclear cell (PMN) phagocytosis (Dosogne et al. 1999). Reports from the same group reported that a decrease in the respiratory burst activity of circulating PMN coincided with an increase in plasma PAG concentrations in cattle (Hoeben et al. 2000). Experimental evidence from this group also demonstrated that treatment of immune cells with PAGs decreased the proliferative ability of bovine erythroid and myeloid cells (granulocytes and monocytes) in vitro, suggesting that high concentrations of circulating PAGs around parturition could contribute to a decrease in innate immune responses and an increased susceptibility of cattle to mastitis (Hoeben et al. 1999). PAGs have also been linked to chemokine release from the endometrium. An early study identified an $8 \mathrm{kDa}$ protein that was secreted by bovine

endometrial explants in response to treatment with PSPB and IFN (Austin et al. 1999; T. R. Hansen et al. 1999). This protein was identified as bovine granulocyte chemotactic protein-2 (GCP2). It is an alpha chemokine, also known as CXCL6, whose primary function is to recruit and activate neutrophils (Rajarathnam et al. 2019). In the current bovine genome build, this chemokine is now named CXCL5 as it more closely resembles human CXCL5 (more on this point in the 'Immune system' section of this review). More current research from our lab supports the results seen by Austin et al.; bovine endometrial explants treated with boPAGs for 24 and 96 hours showed a significant upregulation in CXCL5 Mrna expression (R. M. Wallace et al. 2019). While the role of neutrophils during early pregnancy is not well studied in ruminants, current evidence for potential immunosuppressive and immune recruitment functions of the PAGs may indicate a role in regulating immunity and inflammation associated with attachment in ruminants. 
Several alpha chemokines, such as CXCL5, not only recruit neutrophils to sites of inflammation but are notable players in tissue angiogenesis. Chemokines containing a Glu-Leu-Arg (ELR) amino acid motif have been shown to promote angiogenesis in vitro and in vivo (Strieter et al. 1995; Balestrieri et al. 2008). Angiogenesis is very important in the endometrium and in the developing placenta for facilitating transplacental exchange (Reynolds and Redmer 1995 and 2001). Since boPAGs can upregulate transcription and production of endometrial $\mathrm{CXCL5}$, it may be likely that this function is not only important for immune regulation but also angiogenesis of the endometrium. Results from the current research of this thesis demonstrate that PAGs can induce an increase in expression of several angiogenic chemokines ( $C X C L 1, C X C L 2$, and CXCL5), providing further support for this hypothesis. The role of chemokines in angiogenesis will be further discussed in the 'Immune system' section of this review.

Uterine serpin is another immunomodulatory protein that has been shown to interact with PAGs (Mathialagan and Hansen 1996). The serpins are a large family of serine proteinase inhibitors. One member of this family, in particular, is expressed in the endometrium of some mammals. Uterine serpin, also known as SERPINA14, is a serpin that is regulated by progesterone and appears to have lost its inhibitory activity towards serine proteinases (Padua and Hansen 2010). It is thought that the function of SERPINA14 may be species specific; in sheep it has been shown to have immunomodulatory properties. Ovine SERPINA14 can block lymphocyte proliferation induced by IL-2 (Skopets, Liu, and Hansen 1995; Peltier, Liu, and Hansen 2000), block ovine and murine NK cell activity (W.-J. Liu and Hansen 1993), and reduce antibody production in ewes immunized against a T-cell dependent antigen (Skopets, Liu, and Hansen 1995). While SERPINA14 does not bind to serine proteinases, it does have an affinity for aspartic proteinases and can weakly inhibit pepsin A and C. It can also bind ovine PAGs (Mathialagan and Hansen 1996). Circumstantial evidence in bovine endometrium demonstrated that endometrial expression of SERPINA14 and boPAG1 and -2 was inversely related. In cows artificially 
infected with $N$. caninum, endometrial boPAG1 and -2 expression was elevated while SERPINA14 expression was reduced (Serrano-Pérez et al. 2016). Due to the fact that PAGs are produced by the placenta, measuring PAG Mrna expression in the endometrium usually means that separation of the fetal membranes from the endometrium was incomplete and there are still trophoblasts left over in the endometrium. That being said, PAG Mrna was consistently upregulated in the endometrium of $N$. caninum infected animals, perhaps suggesting an increase in trophoblast PAG expression and deposition in the endometrium in response to the infection (Serrano-Pérez et al. 2016). Increasing PAG expression coupled with decreasing SERPINA14 expression could signify an inverse or antagonistic relationship between these two proteins. If an active infection is present it would be counterintuitive to increase production of an immunosuppressant such as SERPINA14. Perhaps the PAGs both decrease immunosuppressive proteins and increase immune cell recruitment via chemokine signaling. Of course this is speculative and more research will need to be done to conclude this.

Lastly, circumstantial evidence may suggest that the circulating PAGs may modulate circulating immune cell activity. In the cow, systemic PAG concentrations peak around parturition (Green et al. 2005a) but circulating PMN activity is lowest around parturition (Kehrli et al. 1989; Saad et al. 1989; P. J. Hansen 2013). Immune cells treated with boPAGs in vitro showed decreased proliferative capacities of erythroid and myeloid lineages. If this also occurs in vivo then it might imply a role for the PAGs in the maternal circulation in immune tolerance and acceptance of the fetus (Hoeben et al. 1999). However, reduction of systemic PAGs in sheep through autoimmunization did not result in fetal rejection (Egen et al. 2009).

There is still quite a bit that is unknown about the PAG family, the biggest mystery being their function(s). Some of the immunomodulatory data for the PAGs seems conflicting, which further adds to the growing complexity of this family of placental enzymes. Are the PAGs inhibiting immune cell function or are they recruiting immune cells to the site of attachment through chemokine signaling? 
Perhaps the chemokines are not recruiting immune cells at all, rather are they promoting angiogenesis instead. Are the PAGs helping to promote maternal tolerance to the semi-allogenic fetus? It is most likely that the answer is multifactorial. It is also possible that there are functional differences between the individual PAGs and even between species. The majority of research related to PAG function has been conducted in vitro, so perhaps more in vivo research is needed to understand the role of the PAGs during pregnancy.

\section{Immune system}

\section{Overview}

The first record of immunological observation is believed to date all the way back to $430 \mathrm{BC}$ after a plague epidemic in Athens, a Greek historian noted that those who survived did not catch the disease a second time (Retief and Cilliers 1998). Many years later, Louis Pasteur postulated that disease is caused by unseen germs (Plotkin 2005). In contrast to popular belief at the time, this was shortly after proven to be true by Robert Koch, who went on to discovered several infections agents such as tuberculosis, cholera, and anthrax (King 1952). Pasteur and Koch are regarded as the fathers of modern

immunology because their observations and discoveries set the groundwork for the characterization and study of the immune system (Sattler 2017). Today, we know the immune system as a biological system whose primary roles are to control inflammation and to prevent infection from harmful agents such as bacteria and viruses. It has to ability to distinguish 'self' from 'non-self'. This means that the material and/or microorganisms that make up the body and its many physiological systems (i. e. self) are tolerated, while those that are foreign (non-self) are quickly eliminated. This way, the immune system provides us with rapid, specific, and protective responses towards the many potentially pathogenic microorganism/substances that inhabit the world around us (Parkin and Cohen 2001; Paul 2003; De and Tomar 2014; Sattler 2017). 
The immune system is comprised of many specialized cells collectively referred to as leukocytes. Leukocytes are all derived from hematopoietic stem cells in the bone marrow. Hematopoietic stem cells can differentiate into two types of progenitor cells that give rise to the two main classes of immune cells: myeloid and lymphoid. Myeloid progenitor cells further differentiate into many different cell types such as erythrocytes, megakaryocytes, mast cells, granulocytes, and monocytes. Lymphoid progenitor cells further differentiate into T-cells, B-cells, and natural killer (NK) cells (Huston 1997). Further descriptions of these two cell lineages (myeloid and lymphoid) will be discussed in more detail in the following sections of this review.

Immunity can be broken down into 2 main branches: innate and adaptive immunity. Innate immunity is considered to be phylogenetically more ancient than the adaptive immune system and is found in all plants and animals (De and Tomar 2014). Chaplin defined the innate immune system as "all aspects of the host's immune defense mechanisms that are encoded in their mature functional forms by the germ-line genes of the host" (Chaplin 2010). This includes physical barriers such as epithelial and mucosal barriers (such as those in the respiratory and digestive tracts), soluble proteins and other small molecules present in biological fluids (such as complement proteins), cytokines and chemokines, effector cells, and membrane bound receptors that bind pathogenic antigens (Chaplin 2010; Aristizábal and González 2013). The effector cells of the innate immune system are the first responders to sites of damage or infection. They act first because they don't require much, if any, time for terminal differentiation or clonal expansion in response to an immune stimulus, unlike the cells of the adaptive immune system. These include granulocytes, monocytes, dendritic cells, and NK cells (Medzhitov and Janeway 2009). Together all of these components work to control and contain infection and inflammation until the adaptive immune system is able to step in.

The adaptive immune system is made up primarily of lymphocytes, i.e. T-cells and B-cells. NK cells can also be considered an adaptive immune cell, though they possess qualities of both innate and adaptive 
immunity. The adaptive immune system is activated by the innate immune system. Cells from the innate immune system present processed antigens to immature T-cells and B-cells within secondary lymphoid organs, primarily lymph nodes. Binding of the antigen to T-cell receptors (TCR) and B-cell receptors (BCR) activates the naïve lymphocyte and initiates a process of activation, differentiation, proliferation, and clonal expansion (Parkin and Cohen 2001; Medzhitov and Janeway 2009; Takada and Jameson 2009; Sun et al. 2020). The result is generation of a large population of lymphocytes that express cell surface receptors or produce antibodies that specifically target the foreign antigen. This process can take three to seven days to generate sufficient numbers of clones for an efficient immune response. Because of this, the adaptive immune response is slower than the innate response. However, the response generated is highly specific (Parkin and Cohen 2001; F. Liu and Whitton 2005; Kurtulus, Tripathi, and Hildeman 2013; De and Tomar 2014).

\section{Lymphoid cell origins}

Cells of the lymphoid lineage primarily consist of T-cells and B-cells. These cells are collectively called lymphocytes because of their lymphoid origin. Lymphocytes contribute specificity to the immune system. They possess receptors that are specifically made to target a single antigen, and through the process of clonal expansion, they can create thousands of copies of cells that express a single receptor specific for a single antigen (Adams et al. 2020). The ability to produce a large number of cells with high antigen specificities makes lymphocytes a very powerful too for the immune system in fighting potentially pathogenic substances.

Lymphopoiesis - the generation of lymphocytes - starts in the bone marrow. To make mammalian T-cells, hematopoietic stem cells (HSCs) in the bone marrow first differentiate into lymphoid progenitor cells. A subset of lymphoid progenitors that will be destined to become T-cells migrate to the thymus as thymocytes, where maturation and selection of T-cells takes place (Parkin and Cohen 2001; 
Paul 2003; Kumar, Connors, and Farber 2018). Once in the thymus they undergo a series of complex rearrangements and splicing of cell surface receptors. First, the CD4-CD8 thymocytes begin expression of these receptors to become $\mathrm{CD} 4^{+} \mathrm{CD} 8^{+}$'double positive' cells. The double positive cells then undergo a selection process that ultimately results in T-cells $\mathrm{CD} 4^{+}$or $\mathrm{CD} 8^{+}$'single positive' cells that also co-express several other receptors such as CD45, CCR7, CD25, and Foxp3 (Hori, Nomura, and Sakaguchi 2003; Watanabe et al. 2005; Seddiki et al. 2006; Kumar, Connors, and Farber 2018). The single positive cells then leave the thymus to take up residency in tissues or lymph nodes throughout the body. As previously mentioned, in the lymph nodes, antigen presenting cells can display pieces of processed antigens to the resident T-cells. The MHC-II-antigen-TCR complex activates the naïve T-cell and starts the process of maturation and clonal expansion (K. Takada and Jameson 2009; L. Sun et al. 2020). In most, if not all mammals, this process starts in utero. By the time humans are born they already have a full complement of T-cells sufficient for anti-pathogenic immunity. In mice, T-cells don't begin to populate the lymph nodes until the very end of gestation (Burt 2013).

The other major lymphoid cells are B-cells. Like T-cells, B-cell development starts in the bone marrow where HSCs differentiate into lymphoid progenitor cells. However, unlike T-cells, B-cells derived from the same common lymphoid progenitor cells do not migrate out of the bone marrow. Instead, cell surface receptor rearrangement, splicing, and cellular maturation takes place in the bone marrow (Hardy and Hayakawa 2001). Early B-cell precursors can be identified by expression of the B220 isoform of CD45 and a lack of expression of CD19, a molecule whose expression characterizes all mature B-cells (Li et al. 1996; Allman, Li, and Hardy 1999; Ogawa et al. 2000). Early B-cells also have little or no immunoglobulin rearrangement. At this stage they are referred to as Pro-B-cells or pre-Pro-B-cells (Hardy et al. 1991; Osmond et al. 1998). As the Pro-B-cells mature the BCR is assembled and the process of selection starts where only cells with BCR that are non-reactive towards self are allowed to live (Goodnow et al. 1988; Hartley et al. 1991; 1993). At this stage the B-cells can be recognized by 
expression of cell surface immunoglobulin. When the BCR are fully assembled and autoreactive B-cells have been removed, the now mature B-cell can enter the circulation to take residence in the peripheral tissues and secondary lymphoid organs (Richard R. Hardy and Hayakawa 2001).

\section{Myeloid cell origins}

The other cell lineage derived from HSCs and that constitute the largest percentage of the total immune cells in the body is the myeloid lineage. Myeloid immune cells consist of monocytes and granulocytes. Erythrocytes and megakaryocytes are also derived from the myeloid lineage. Monocytes further differentiate into macrophages and dendritic cells. These cells are generally a little larger than the other leukocytes and have a large bean-shaped nucleus. Monocytes and dendritic cells are referred to as professional antigen presenting cells because their main job in the immune system is to process and present antigens to lymphocytes in order to stimulate the adaptive immune response (Rodgers and Rich 2013). Granulocytes are a group of myeloid cells so named for their characteristic cytoplasmic granules. This group consists of neutrophils, basophils, eosinophils, and mast cells. The granulocytes are considered to be 'first responders' to sites of infection. They provide a fast and efficient defense against an infectious agent and keep the infection under control until the adaptive response can take over (Parkin and Cohen 2001; Kawamoto and Minato 2004).

Like lymphocytes, myeloid cells originate from HSCs in the bone marrow. HSCs can produce myeloid progenitor cells which can differentiate into the many members of the myeloid lineage. Hematopoiesis - the formation of the cellular components of the blood - first occurs during embryonic development and continues throughout the life of the animal. The earliest waves of hematopoiesis during embryogenesis appear to be largely regulated by the transcription factors Gata1 and Pu.1 (Cantor and Orkin 2002; Cantor, Katz, and Orkin 2002). Gata1 is known for its major role in regulating erythropoiesis (formation of erythrocytes) but it is also known to be a negative regulator of myeloid cell 
fate. Gata1 knockdown in zebrafish resulted in a hematopoietic switch to myeloid lineage (Rhodes et al. 2005). In contrast, Pu.1 is a positive regulator of myeloid cell fate (Scott et al. 1994). Later waves of hematopoiesis (later in gestation and in the mature animal) are regulated by several other transcription factors such as Runx1 (Q. Wang et al. 1996), Wnt (Luis et al. 2011; 2012), and Notch (Guruharsha et al. 2012). Though it is currently not fully understood what determines the lineage commitment of the HSCs, it is predicted to be a combination of transcription factors and the tissue microenvironment (Kawamoto and Minato 2004). Other factors such as soluble cytokines and chemokines are believed to be the main drivers of terminal differentiation of the myeloid progenitor cells (Metcalf 1998). For example, cytokines such as G-CSF and M-CSF are important in maintaining homeostatic myelopoiesis (the formation of myeloid cells). Cytokines produced by T-cells, such as IL-3 and GM-CSF, are also crucial in maintaining myelopoiesis.

\section{Role of chemokines}

While the cellular component of the immune system is critical for the recognition and destruction of potentially harmful pathogens, secreted proteins from the immune cells and the surrounding tissues are just as crucial for the initiation and coordination of the immune response. One such family of secreted proteins is the chemokine family. Chemokines are part of a family of small 8-10 kDa chemotactic cytokines (hence the name 'chemokine') that play important roles in immune cell chemotaxis, activation, and in angiogenesis. There have been about 50 chemokines and 20 chemokine receptors identified in humans and mice (Mackay 2001). Chemokines have been well studied in humans and mice and have been sought after as potential therapeutic targets for various diseases, especially cancer (Gangur et al. 2002; Strieter et al. 2006). Studying chemokine functions can be difficult because of the promiscuous nature of the ligands and their receptors. A single chemokine may bind two or three different chemokine receptors. Likewise, a single chemokine receptor may be able to bind several different chemokines (Le et al. 2004). However, ruminants chemokine families and their functions have 
not been as extensively studied. To date, there have been about 40 chemokines identified in cattle and they have been named based on their closest human homolog (Widdison and Coffey 2011). Assigning functions to the bovine chemokines based on amino acid homology is tricky, though. There has been little study of the direct biological roles of the bovine chemokines. Most of the functional research is circumstantial, using experimentally infected animals and measuring changes in immune markers, including chemokines (Santos et al. 2002; Buza et al. 2003; Zhang et al. 2003; Taubert et al. 2006). Phylogenetic and syntenic comparison of the bovine and human chemokine genome would suggest that bovine-specific gene duplication and/or mutations could result in chemokines with high sequence homology but very different functions (Widdison and Coffey 2011). However, there is some direct evidence that suggests some chemokines may indeed be functional homologs of their human counterparts (Rainard et al. 2008; Behrendt et al. 2008). Without further research into the direct functionality of the bovine chemokines, it is unwise to assume they are direct functional homologs of the human genes.

Chemokines fall into three main classes, CC (beta chemokines), CXC (alpha chemokines), and $\mathrm{CX}_{3} \mathrm{C}$ (gamma chemokines), based on the arrangement of two conserved $\mathrm{N}$-terminal cysteine residues, where the $\mathrm{X}$ represents any amino acid residue other than cysteine. The CXC chemokines can be further grouped based on the presence of the previously mentioned ELR amino acid motif preceding the first $\mathrm{N}$ terminal cysteine. CXC chemokines that contain the ELR motif (ELR+) are known for recruiting neutrophils to sites of inflammation (Kobayashi 2008; Rajarathnam et al. 2019) as well as for promoting angiogenesis (Strieter et al. 2006a; Balestrieri et al. 2008). These include CXCL1, -2, -3, -5, -6, -7, and -8. Interestingly, most species only possess a CXCL5 or CXCL6 gene but not both. Humans and non-human primates appear to be the only species who have both genes. Phylogenetic analysis would suggest that a duplication event occurred post-speciation resulting in two duplicate genes in the human genome. All of the ELR+ CXC chemokines bind to the chemokine receptors CXCR1 and/or CXCR2 (Addison et al. 
2000). This appears to be the basis for their neutrophil chemotactic properties because neutrophils express both of these receptors. Binding of an ELR+ chemokine to CXCR1 or CXCR2 on the surface of neutrophils activates G-protein and $\beta$-arrestin-mediated second messenger cascades (Richardson et al. 1998; 2003; Barlic et al. 2000; Molteni et al. 2009). Chemokine-activated PLC and Rac1 in neutrophils induces changes in actin dynamics that are crucial for formation of the leading edge of neutrophils during cellular migration (Hirayama et al. 2007; Xu et al. 2015; Sun et al. 2007). In regards to the role of ELR+ chemokines in angiogenesis, the ELR motif seems to be the key. A very elegant study by Strieter et al. demonstrated this by using a corneal micropocket angiogenesis assay. Rat corneas treated with recombinant CXCL5 or CXCL8 exhibited a significant increase in angiogenesis while corneas treated with CXCL9 or CXCL10 (non-ELR containing CXC chemokines) exhibited little to no angiogenic response. When only the ELR motif of CXCL8 was mutated to TVR (the amino acid sequence preceding the Nterminal cysteine of CXCL10), little to no angiogenesis was initiated. Additionally, when CXCL9 was mutated to contain the $\mathrm{N}$-terminal ELR motif, it was able to induce angiogenesis similar to that of CXCL5 and CXCL8 (Strieter et al. 1995). These results demonstrate that the angiogenic activity of the CXC chemokines is likely to be dictated by the presence or absence of the ELR motif.

CXC chemokines that lack the ELR motif (ELR-) are generally not chemotactic towards neutrophils and some of them possess angiostatic activity (Belperio et al. 2000; Balestrieri et al. 2008). CXCL12 is an exception to this rule, however. CXCL12 is an ELR- chemokine however it plays important roles in chemotaxis and promoting angiogenesis (Kijowski et al. 2001; Salcedo and Oppenheim 2003; Bachelder et al. 2002). Three of the ELR- CXC chemokines, CXCL9, -10 , and -11, are known to be induced by interferons, namely IFN $\gamma$, and are predicted to promote Th1/type 1 cytokine-mediated immune responses (Sharma et al. 2003; Hillinger et al. 2003). CXCL9, -10, and -11 not only promote angiostasis but are potent anti-angiogenic molecules. CXCL9 and CXCL10 can actively inhibit the angiogenic effects of the ELR+ CXCL5 and CXCL8 (Strieter et al. 1995). In corneal micropocket angiogenesis assays, 
treatment with CXCL5 or CXCL8 (ELR+) together with CXCL9 or CXCL10 resulted in little to no angiogenic response after 6 days. This is in stark contrast to the angiogenic response seen with ELR+ chemokines alone (Strieter et al. 1995). The angiostatic/anti-angiogenic activity of these chemokines is mediated through the receptor CXCR3 (Lasagni et al. 2003; Kelsen et al. 2004). The ELR- CXC chemokines are also chemotactic towards several leukocytes, primarily mononuclear leukocytes such as T-cells, B-cells, macrophages, and NK cells. Chemotaxis of these cells is mediated through CXCR3, CXCR4, and CXCR5 signaling, depending on the chemokine (Griffith, Sokol, and Luster 2014). CXCL12 is known for its crucial role in regulating hematopoiesis and retention of immune cells in the bone marrow (Moser and Loetscher 2001). CXCL12- or CXCR4 (the receptor for CXCL12)-deficient mice die perinatally due to deficient B lymphopoiesis and myelopoiesis (Ma et al. 1998). Overall, the CXC chemokines serve many roles in the body that are important not only for immune system responses but also for tissue homeostasis. Maintaining a balance between innate and adaptive immune cell recruitment as well as between angiogenesis and angiostasis is important in maintaining homeostasis throughout the body.

The CC chemokine family is quite large in humans, consisting of about 27 individual chemokines. They can be group into 2 main gene clusters: the monocyte chemoattractant protein (MCP) cluster and the macrophage inflammatory protein (MIP) cluster (Widdison and Coffey 2011). The other members of the CC chemokine family that do not fall into these two clusters don't cluster with any other CC chemokine upon phylogenetic analysis. These chemokines likely represent an evolutionarily older group of chemokines because they are generally pretty conserved across species (Widdison and Coffey 2011). The MCP cluster consists of chemokines CCL2 (MCP-1), CCL7 (MCP-3), CCL8 (MCP-2), and -13 (MCP-4). CCL11 (eotaxin), despite not being a MCP, also falls into this cluster because it shares about $70 \%$ amino acid identity with the other MCP members (Luster and Rothenberg 1997). The characteristic feature of this group of CC chemokines is their ability to recruit monocytes and T-cells. All MCP-clustered CC chemokines (except CCL11) bind the receptor CCR2. When monocytes leave the bone marrow they can 
further differentiate into anti- or pro-inflammatory monocytes that express $\mathrm{CX}_{3} \mathrm{CR} 1$ and $\mathrm{CCR} 2$, respectively (Geissmann et al. 2003). CCL2, $-7,-8$, and -13 likely aid in the inflammatory process by recruiting CCR2 ${ }^{+}$pro-inflammatory monocytes. CCL2 and CCL8 have been studied in the ovary and have been implicated in the process of luteinization and/or $\mathrm{CL}$ regression by recruiting pro-inflammatory monocytes to clear the cellular debris after follicular rupture or during luteolysis, respectively (Tsai et al. 1997; Haworth et al. 1998; Duffy et al. 2019). CCL11 is the one member of the MCP cluster that does not recruit monocytes or lymphocytes. CCL11, also known as eotaxin, is responsible for promoting residence of eosinophils and basophils in peripheral tissues and promoting their release from the bone marrow (Palframan et al. 1998; Mishra et al. 1999) The other cluster of CC chemokines is called the macrophage inflammatory protein (MIP) cluster. This cluster consists of CCL3, $-4,-5,-6$ (mouse only), $14,-15,-16,-18$, and -23 . This group of chemokines are primarily responsible for recruiting macrophages, NK cells, and T-cells to sites of inflammation. These chemokines serve many roles not just in inflammation but also in tissue homeostasis. For example, CCL3 and CCL5 are constitutively expressed in lymphoid tissues, however, CCL3, CCL4, and CCL5 are all implicated in fighting HIV infections (Cocchi et al. 1995; Baggiolini 1998).

It is clear that chemokines play important roles in various processes throughout the body. Because of the large number of them and their receptor promiscuity, there is still quite a bit that is unknown of chemokines. Differences between species only adds an additional layer of complexity to this already complex system of immunomodulators.

\section{Inflammation, ovulation, and pregnancy}

\section{Role of the immune system during ovulation and luteinization}

As previously described, the immune system is pivotal in protecting the body from the many threats posed by our environment. However, the immune system does far more than simply fight 
infections. It is crucial for many homeostatic processes throughout the body. Leukocytes are constantly surveying the tissue environment and help to aid in removal of cellular debris during tissue remodeling and after apoptosis. Two processes that requires rapid modeling and cellular turnover are the processes of ovulation and luteinization. Ovulation can be defined as the rupture of the ovarian follicle for the release of a mature oocyte into the oviduct. During ovulation, the pre-ovulatory follicle wall undergoes proteolytic degradation that results in rupture and release of the oocyte. Immediately following follicular rupture, the follicle is infiltrated with new vasculature and the remaining follicular cells are reorganized to begin the process of luteinization (Espey 1967; Smith et al. 2002; Duffy et al. 2019).

In the pre-ovulatory ovarian follicle, the ovarian vasculature normally delivers circulating leukocytes, such as macrophages and neutrophils, to the ovarian tissue, some of which take up residency in the ovary. During follicular rupture, the secretions of cytokines and chemokines from the follicular cells and the resident immune cells induces an acute inflammatory response that, coupled with proteolytic activity, likely causes weakening of the follicular wall and rupture (Brännström and Enskog 2002; Duffy et al. 2019). There is a lot of evidence suggesting that immune infiltration of the ovarian follicle is not necessary for ovulation but rather are facilitators of ovulation. In vitro, rabbit and rat ovaries perfused with LH were still able to ovulate, but the efficiency was decreased (Brännström et al. 1988; Pall et al. 2000; Viana et al. 2011). While this does not rule out resident immune cells as contributors to ovulation, it may suggest that, without influx of circulating immune cells, full ovulatory efficiency cannot be reached. This is supported by data of immune supplementation of in vitro LHperfused rat ovaries that showed increased ovulation rates (Hellberg et al. 1991). It's likely that the immune cells are performing multiple roles in the ovary during ovulation. Many leukocytes secrete proteases, such as MMPs, so it may be that the immune cells are facilitating matrix remodeling in the ovary (Webster and Crowe 2006). Immune cell protease substrates are not restricted to matrix proteins, however, but also include cytokines, chemokines, cell surface receptors, and adhesions molecules. This 
would indicate their involvement not only in the matrix remodeling but in signal activation as well (Duffy et al. 2019). Additionally, monocytes, macrophages, and neutrophils (immune cells found in ovarian follicles) are all known to secrete angiogenic factors such as VEGF. Angiogenesis is a critical part of inflammation and ovulation so it's possible that the resident or invading immune cells are facilitating follicular angiogenesis (Gargett and Rogers 2001; Guimerà et al. 2009; Heissig et al. 2010).

There are several chemokines that are upregulated in ovarian tissues during ovulation. In the bovine ovary, CXCL8 levels are usually low. After the LH surge, CXCL8 production by the granulosa and theca cells of the ovary increases dramatically (Bukulmez and Arici 2000). As previously mentioned in this review, CXCL8 is not only a pro-angiogenic chemokine but it also is a potent neutrophil chemoattractant. Furthermore, neutralization of either CXCL8 or neutrophils with neutralizing antibodies reduces ovulation rate in rabbits (Ujioka et al. 1998). This clearly demonstrates the importance of the interplay between the follicular cells and the immune system. Other chemokines are upregulated in the ovulatory follicle including CCL20, CXCL12, and CCL2 (Kryczek et al. 2005; DahmKähler et al. 2009; Al-Alem et al. 2015). Each of these chemokines recruits a different subset of leukocytes and CXCL12, in particular, is known to for being a pro-angiogenic chemokine. Taken together, these results demonstrate the intimate communication between the ovary and the immune system and the importance of the immune system in ovulation.

\section{Influence of progesterone on immune system}

During pregnancy, progesterone predominates as the main ovarian steroid hormone influencing the uterine environment. Progesterone is not only important for pregnancy maintenance but it is also a key regulator of immune function in the endometrium. Experiments placing skin allografts within the uterine lumen showed that treatment with progesterone can delay resorption of the allograft (Hansen et al. 1986). Progesterone can also reduce the number of endometrial lymphocytes in sheep. Analysis 
of endometrial leukocyte populations in ovariectomized ewes treated with progesterone showed that treated ewes has significantly fewer CD45R+ lymphocytes at all time points (Gottshall and Hansen 1992). In vitro experiments have demonstrated that progesterone can directly inhibit activation and proliferation of ovine peripheral blood lymphocytes (L. D. Staples, Binns, and Heap 1983; Linton D. Staples et al. 1984; Monterroso and Hansen 1993). There is conflicting evidence as to whether progesterone can act as an immunosuppressant in nonuterine sites. Ewes experimentally infected with the parasitic worms Haemonchus contortus and treated with $25 \mathrm{mg}$ of progesterone daily exhibited an increase in load of adult and larval worms and a decreased proliferative response of peripheral blood lymphocytes to concanavalin A compared to the untreated controls (Fleming and Gamble 1993). One study did not observe any differences in peripheral lymphocyte proliferation between pregnant and nonpregnant ewes (Miyasaka and McCullagh 1982) while another study did record a reduced proliferative response during pregnancy (Rai-el-Balhaa et al. 1987). Whether progesterone has systemic immunosuppressive effects is unclear. However, progesterone does have local immunosuppressive/immunomodulatory effects in the uterus during pregnancy.

\section{Role of immune system for establishment of pregnancy}

Immune regulation in the uterus is crucial for the establishment and maintenance of pregnancy. Over 60 years ago, Sir Peter Medawar proposed that the presence of the maternal immune system at the implantation site was the key to understanding why the semi-allogenic fetus is not rejected by the maternal system (Medawar 1948 and 1952). This began the pursuit to understand the roles of the maternal immune system throughout pregnancy. Today, we still don't have a clear answer as to why the fetus and placenta are not rejected and destroyed by the maternal immune system. The common theory for many years was that pregnancy induced an immunosuppressed state in the mother and that was the reason the fetus was not rejected. This theory has gradually been disproven and we now know this to be incorrect. The popular theory today is that pregnancy does not suppress the maternal 
immune system, instead it induces a more tolerogenic immune state (Mor et al. 2011). The true answer is most likely multifaceted and more than likely differs between species. In humans it has been proposed that placental trophoblasts may be able to induce differentiation of immune cells into a trophoblast-supporting phenotype (Mor et al. 2011). It has also been proposed that expression of classical and non-classical MHC molecules on the trophoblasts allows them to evade immunological attack (Papúchová et al. 2019; Xu et al. 2020).

In ruminants, little is known about how the maternal immune system responds to the attaching conceptus. It is thought that IFNt may be responsible for priming the uterine microenvironment and maternal immune system for support of the developing placenta (Ott and Gifford 2010; Nagaoka et al. ; Oliveira et al. 2013; Oliveira and Hansen 2008; Kamat et al. 2016). As previously discussed, progesterone dominance during attachment and it's immunosuppressive actions in the uterus could also be another mechanism for immune tolerance. INFT can upregulate the expression of chemokines CCL2 and CCL8 in the pregnant endometrium of cattle, both of which are known to be chemotactic for monocytes and macrophages. Additionally, an expansion of monocytes and dendritic cells in the bovine endometrial stroma has been recorded as early as day 13 of pregnancy (Mansouri-Attia et al. 2012). In mice, CCL2 has been suggested to stimulate Th2-type responses (Gu et al. 1997), so perhaps the increase in CCL2 and CCL8 expression during bovine pregnancy is a consequence of monocyte and macrophage invasion and is augmented by IFN $\tau$ to promote an anti-inflammatory Th2-type immune response. IFNT has also been shown to upregulate endometrial expression of CXCL10, which is also chemotactic for macrophages (Nagaoka et al. 2003b). Endometrial CXCL10 has also been implicated in promoting trophoblast adhesion in cattle and sheep (Nagaoka et al. 2003a; Imakawa et al. 2006) This would further support the idea that interferon Tau is modulating the uterine immune environment to promote attachment and pregnancy. IFN may also modulate systemic immune cells in cattle. There is a pregnancy-associated increased in expression of interferon stimulated genes such as ISG15, Mx1 and 
Mx2 in peripheral blood leukocytes as early as day 16 of pregnancy (Gifford et al. 2007b). Because this is during the window of MRP and INFT secretion by the trophoblasts in cattle, it is believed that IFN may be acting systemically to modulate the maternal immune response. Additionally, the influx of macrophages and dendritic cells in the bovine endometrium is most likely in response to paternal antigens and MHC-I molecules on the pre- and peri-attachment conceptus (Doyle et al. 2009). It's possible that the cytokine and chemokine profiles created in the endometrium by IFN conceptus-derived products can drive differentiation of the invading macrophages towards the M2 activation pathway, which can decrease the activation of anti-conceptus immune responses (Oliveira et al. 2010).

In addition to macrophage infiltration, an increase in NK cell infiltration into the endometrium occurs during early pregnancy in cattle (Oliveira et al. 2013). In other species, NK cells make up a large population of the endometrial leukocyte population during implantation (Dietl et al. 2006; Hanna et al. 2006). In mice, NK cells have been shown to be crucial players in promoting fetal growth and development. The absence of maternal uterine NK cells during pregnancy in mice resulted in impaired fetal growth. Replacement of uterine NK cells from a normal individual was able to restore this phenotype (Fu et al. 2017). However, the role of uterine NK cells in cattle is still unclear. In mice and humans it is believed that uterine NK cells may be promoting vascular remodeling via the production of growth factors and angiogenic factors (Mor et al. 2011; Munoz-Suano, Hamilton, and Betz 2011). It's possible that NK cells in the bovine endometrium may be playing a similar role. However more research is needed to further explore this theory.

Overall, the immune system during early pregnancy is not suppressed as was once believed. Rather, the immune response appears to be modulated to perhaps promote a more protective environment for the conceptus. Research in the field of reproductive immunology is ongoing. 
More insights into the maternal immune status during pregnancy are being gained with each study. The focus of the research of this thesis will focus on the potential roll of the PAGs as immunomodulators in the endometrium of early pregnancy. The focus will be primarily on the ability of the bovine PAGs to alter gene transcription of chemokines in the bovine endometrium. 


\section{CHAPTER II}

\section{EFFECTS OF BOVINE PREGNANCY-ASSOCIATED GLYCOPROTEINS ON GENE TRANSCRIPTION IN BOVINE ENDOMETRIAL EXPLANTS}

\section{ABSTRACT}

Pregnancy-associated glycoproteins (PAGs) are a complex gene family, whose members are expressed by trophoblasts of ruminants and related species. In cattle, the PAGs accumulate at the trophoblast-uterine interface and many can enter the maternal circulation. However, very little is known about the role they play in pregnancy although preliminary results suggest that PAGs at the placenta-uterine interface play roles involving matrix turnover and immune modulation. This study was designed to provide further insight into the biological roles of bovine PAGs by measuring changes in endometrial transcript abundance for some matrix metalloproteinases (MMPs) and chemokines/cytokines. PAGs for these experiments were purified from mid-gestation bovine placental extracts by affinity chromatography. Heifers were synchronized and bred by artificial insemination with high fertility semen $(n=14)$ or dead semen $(n=5)$. Heifers were slaughtered at day 18 postinsemination and the reproductive tracts were obtained and flushed to determine if a conceptus was present. Endometrial explants were collected and split between 4 groups: pregnant with and without $15 \mu \mathrm{g} / \mathrm{ml} \mathrm{PAG}(\mathrm{n}=10)$ and nonpregnant with and without $15 \mu \mathrm{g} / \mathrm{ml}$ PAG $(\mathrm{n}=9)$. Endometrial explants were cultured with or without added PAGs for up to 96 hours at $37^{\circ} \mathrm{C}$ and $5 \% \mathrm{CO}_{2}$ and samples were harvested at 24 hour intervals for extraction of RNA and fixation. This study focuses on the 48 and 72 hour collection points. Transcript abundance for target genes was analyzed in the endometrial tissue by quantitative PCR. The normalization control transcript was peptidylprolyl isomerase A (PPIA). After 48 and 72 hours, significant increases in CXCL1, CXCL2, and CXCL5 as well as MMP1, MMP3 and MMP13 were measured in the PAG-treated endometrium from both pregnant and non-pregnant animals $(\mathrm{P}<0.05)$. CCL11 was upregulated at both time points in the pregnant endometrium but only after 72 hours in the nonpregnant endometrium. There were also significant decreases in message for CCL2, 
CCL8 and CCL16 in the PAG-treated groups from both pregnant and non-pregnant animals at each time point $(\mathrm{P}<0.05)$. Significant decreases in $C X C L 10, C X C L 12$, and Regakine message were seen only in PAGtreated endometrium from pregnant animals $(P<0.05)$. Structural differences in the luminal and glandular epithelium were seen in the PAG-treated biopsies from both non-pregnant and pregnant heifers. These results suggest that PAGs are capable of inducing structural changes as well as changes in transcript abundance in bovine endometrial explants, which suggests that this model system might be useful to assess PAG function at the placenta-uterine interface.

\section{INTRODUCTION}

Establishment of pregnancy is a complex process that depends on a variety of factors taking place in a spatially and temporally synchronized fashion. This includes the establishment of endometrial receptivity, conceptus signaling, hormonal signaling, , maternal immune tolerance and much more. Over the years these factors, which contribute to the establishment of successful pregnancies, have been extensively studied. Although we now know a lot about these processes, there is still much that remains unclear. In cattle, one area that remains a major focus is placental development and function, especially in regard to the release of secretory products and the way in which they interact with maternal tissues. However, the roles that some of these secretory proteins play at the fetal-maternal interface, within the endometrium, or in the maternal system remain unclear. By studying the events of early pregnancy, we can potentially find ways to improve reproductive efficiency in livestock and gain a better mechanistic understanding of the roles of the placenta and its secretory products during pregnancy.

The role of the placenta during pregnancy is multifaceted. It acquires nutrients from maternal tissues, exchanges gasses, eliminates waste and provides protection for the growing fetus. Despite that mammalian placentas all serve the same basic functions, placental structure and extent of trophoblast 
invasiveness varies among species (Roberts et al., 2016). Some of these distinctions are reflected by unique trophoblast forms and gene products that are restricted to certain orders or suborders of species or to certain placental types. An example of these aspects can be found within the ruminant placenta, which is distinct in several ways. One unique feature is the presence of a population of giant binucleated trophoblasts cells. These binucleated cells (BNC) are distinct in that they produce vast amounts of proteins that are packed into dense granules for secretion into maternal tissue (Wooding 1982 and 1992). A major component within these secretory granules is a family of proteins known as the pregnancy-associated glycoproteins (PAG; Wooding et al, 2005). The PAGs are products of a gene family of aspartic proteases produced exclusively by the trophoblasts of most even-toed ungulates and cetaceans within the Artiodactyla order (Xie et al, 1997; Hughes et al, 2000 and 2003).

Over the years the PAGs have been extensively studied. Their temporal and spatial Mrna expression patterns have been well characterized and their protein localization patterns at the interface and in the endometrium have been described as well. However, most of the research focus has been on the presence of PAGs within the maternal circulation during pregnancy. Their accumulation in maternal blood soon after the establishment of a functional chorioallantoic placenta has been used as the basis for pregnancy diagnosis (Zoli et al. 1992; Green et al. 2005). The gestational age at which PAGs become detectable and/or their circulating concentrations earlier in pregnancy can serve as useful markers for predicting embryonic loss (Pohler et al. 2013; 2016).

Despite these efforts, there is little understood about the specific functions being performed by the PAGs during pregnancy. However, several published reports have speculated about their potential roles. In ruminant ungulates, the PAGs can be divided into two distinct groups that have been defined as 'ancient' and 'modern' PAGs based on the times at which they arose during Artiodactyl evolution. The modern PAGs are expressed primarily in BNCs and these PAGs show distinct expression patterns. Most or all of the BNC-specific PAG can enter the maternal circulation. Some of these also accumulate in 
the endometrial connective tissue adjacent to the cotyledonary villi. The ancient PAGs, most of which are produced by the mononucleated trophoblasts, tend to accumulate along the interface (Green et al, 2000; Wooding et al, 2005). Roles related to adhesion and protein turnover in the developing placentome have been proposed (Telugu et al, 2010; Wallace et al, 2019; Wooding et al, 2005). Previous research from our laboratory has demonstrated that PAGs can induce changes in transcript abundance of some matrix-metalloproteinases (MMP) in bovine endometrial explants (Wallace et al, 2019). Other research groups have suggested luteotropic roles for the PAGs. Bovine PAGs have been shown to increase secretion of PGE2 from luteal cells and endometrial tissue in vitro (Weems et al, 2003 and 2007); PGE2 has antiluteolytic and luteotrophic activity. Those PAGs that enter into the maternal circulation exhibit a large periparturient rise in circulating levels (Green et al, 2005), which is suggestive that the PAGs may be functioning to some degree outside of the uterine environment.

Among the speculated roles of the circulating PAGs is the ability to modulate the maternal immune system. The localization of some modern PAGs in the endometrial stroma of caruncular villi would place these proteins in a position to directly influence maternal immune responses (Wooding et al, 2005). Bovine PAG-1 was shown to reduce the proliferative activity of myeloid cells and granulocytes in vitro (Hoeben et al, 1999). Later, Klisch et al. suggested that the glycans on the surface of the bovine PAGs (boPAG) might be able to interact with selectins - adhesion molecules that function in lymphocyte migration - and thereby inhibit selectin-mediated cell adhesion of lymphocytes (Klisch et al. 2006). Furthermore, the PAGs are capable of interacting with uterine serpins - proteins capable of inhibiting proliferation of peripheral blood lymphocytes and natural killer cell activity in vitro (Mathialagan and Hansen, 1996; Peltier et al, 2000; Serrano- Pérez et al, 2016). The PAGs have also been implicated in modulation of immune cell chemotaxis by modulating the expression and release of chemokines - small chemotactic cytokines involved in immune cell recruitment and activation. Of particular interest are a group of chemokines in the C-X-C chemokine family known as the neutrophil activating chemokines. In 
cattle, this group consists of CXCL1, $-2,-3,-5,-7$, and -8 . Bovine pregnancy-specific protein B (PSPB; which is similar or identical to boPAG-1) was shown to induce the secretion of granulocyte chemotactic protein 2, now known as CXCL5 in cattle, in the endometrium in vitro (Austin et al, 1999). Previous research from our lab has demonstrated that PAGs can increase the Mrna abundance of CXCL5 in endometrial explants in vitro (Wallace et al, 2019).

Clearly, the role of the PAGs remains unclear and more research is needed to better define their function. This study was designed to gain more insight into the biological roles of bovine PAGs in the endometrium during pregnancy. Given the research that suggests the PAGs may play a role in immunomodulation, we hypothesized that the application of bovine PAGs to bovine endometrial explants would produce measurable changes in transcript abundance of immune-related proteins. Based on the putative roles of the PAGs mentioned previously, we investigated changes in chemokines known to be involved in immune cell recruitment and modulation. We also investigated the pathways associated with prostaglandin production because prostaglandins not only play an important role in pregnancy but in immune responses as well.

\section{MATERIALS AND METHODS}

\section{Animals and Collection of Tissue}

Angus cross bred heifers $(n=19)$ were synchronized by using a modified 5-Day CO-Synch + CIDR protocol. Heifers received an intravaginal CIDR insert (controlled Internal Drug Release; Eazi-Breed CIDR Insert; Zoetis Animal Health, Kalamazoo, Michigan) containing 1.38g progesterone from day -7 to day -2 with day 0 being the day of insemination. Heifers received $100 \mu \mathrm{g} \mathrm{GnRH}$ (Cystorelin; Merial, Duluth, Georgia) at the time of CIDR insertion. At the time of CIDR removal, heifers received an intramuscular injection of a prostaglandin $\mathrm{F}_{2} \mathrm{a}$ analog (Estrumate; Merck, Summit, New Jersey; equivalent to $1 \mathrm{mg}$ of 
cloprostenol) and were fitted with estrous detection patches (Estrotect; Rockway Inc., Spring Valley, Wisconsin). Heifers were artificially inseminated at a fixed-time (60 hours) after CIDR removal. At the time of breeding, heifers received another injection of $100 \mu \mathrm{g}$ GnRH and were inseminated with high fertility Jersey bull semen. Five heifers were assigned to the control group (dead semen). For the control heifers, semen from the same Jersey bull was left at room temperature for approximately $24 \mathrm{hr}$ to immobilize the sperm. Lack of sperm motility was verified under a microscope before insemination of the control heifers. A second CIDR was inserted in all heifers at day 16 post-insemination to ensure progesterone levels remained elevated in both nonpregnant and pregnant animals at slaughter. On day 18 post-insemination, reproductive tracts were collected from each heifer immediately after slaughter.

\section{Endometrial Explant Culture}

Reproductive tracts from all heifers were collected and transported to the laboratory within 30 minutes after collection. At the abattoir, tracts were placed in a Ziploc bag that was then placed in a second Ziploc bag. In the lab, the outer Ziploc bag was removed and the inner bag rinsed with 10\% bleach followed by sterile saline. The cervix and oviducts of each tract were clamped upon removal from the bag to prevent any outside materials from getting into the tract. Excess tissue was trimmed for ease of handling. The exterior of the tract was sprayed with $70 \%$ ethanol then rinsed with Dulbecco's PBS. Uterine horns were flushed with sterile Dulbecco's PBS and pregnancy was verified by the presence or absence of a conceptus in the flush. The conceptus was immediately snap frozen in liquid nitrogen and stored at $-80^{\circ} \mathrm{C}$ until use. Before opening the tract, the exterior was rinsed once more with $10 \%$ bleach followed by Dulbecco's PBS. The tract was placed on a clean tray and immediately transferred to a laminar flow safety cabinet where all subsequent steps were then carried out. 
Explants, 5-6mm in diameter $(n=20 ; 50-60 \mathrm{mg}$ each), were collected from the horn ipsilateral to the corpus luteum. Due to the early stage of pregnancy and the heifers being nulliparous, caruncular tissue were nearly indistinguishable from intercaruncular tissue. Therefore, no effort was made to restrict explants exclusively to caruncular or intracaruncular tissue. Groups of explants were placed into culture medium with or without added bovine PAGs. Ultimately, four experimental treatment groups were created: 1$)$ nonpregnant + no PAG $(n=9), 2)$ nonpregnant $+15 \mu \mathrm{g} / \mathrm{MI}$ PAG $(n=9), 3)$ pregnant + no PAG $(n=10)$, and 4) pregnant $+15 \mu \mathrm{g} / \mathrm{MI}$ PAG $(\mathrm{n}=10)$. The nonpregnant group contained the heifers bred to dead semen $(n=5)$ as well as the bred heifers that did not have a conceptus present at the time of collection (day 18; $n=4$ ). Endometrial expression of interferon-stimulated genes (IFI6, ISG15, OAS-1) was analyzed in the four bred animals that were lacking a conceptus on day 18 . No differences in the expression of these genes were seen when compared to the control nonpregnant group. This would suggest that they did not conceive or the embryo was lost prior to interferon tau production. These animals were then put into the control group.

Bovine PAGs for this experiment were purified from mid-gestation bovine cotyledonary extracts and evaluated to access quantity and quality (see Appendix I, Appendix II, and Appendix III). Endometrial explants from individual animals were cultured in $100 \times 20 \mathrm{~mm}$ cell culture plates ( $720 \mathrm{mg}$ tissue $/ 20 \mathrm{ml}$ of media) at $37^{\circ} \mathrm{C}$ under $5 \% \mathrm{CO}_{2}$ with constant movement on a slowly rocking platform. The culture medium was made up of Dulbecco's Modified Eagle Medium with 4.5g/L D-glucose (Invitrogen, Grand Island, New York), 0.1\% pure bovine serum albumin, 1\% L-glutamine (Gibco, Grand Island, New York, 15 antibiotic-antimycotic (Gibco, Grand Island, New York), 10\% heat treated horse serum (Gibco, Grand Island, New York), progesterone (10ng/MI), and estradiol (5pg/MI).

Four pieces of tissue were collected prior to incubation ( 0 hour) and after 24, 48, 72, and 96 hours of culture. The collected samples were stored at $-20^{\circ} \mathrm{C}$ in RNAlater ${ }^{\mathrm{TM}}$ (Invitrogen; 3 replicates per heifer per experimental group) or in $10 \%$ Neutral Buffered Formalin at $4^{\circ} \mathrm{C}$ ( 1 replicate per heifer per 
experimental group) for RNA isolation and histology, respectively. At the 48 -hour time point half of the spent media was removed and replenished. Aliquots of the media (2MI) were collected at the 48 - and 96-hour time points and stored at $-80^{\circ} \mathrm{C}$.

\section{RNA Isolation, Reverse Transcription, and Primer Validation}

Total RNA was extracted from one endometrial tissue sample per heifer per experimental group using the Rneasy Mini Kit (Qiagen, Valencia, California) with On-Column Dnase Digestion according to the manufacturer's instructions. The following adjustment was made to the protocol: samples were minced by hand with two 10-gauge scalpels or homogenized using a GentleMACS Dissociator (Miltenyi Biotec, Bergisch Gladbach, Germany). The concentration and purity of RNA samples were determined using spectrophotometry at 260 and $280 \mathrm{~nm}$.

Complementary DNA was synthesized from $0.5 \mu \mathrm{g}$ total RNA by using the PrimeScript $1^{\text {st }}$ Strand Cdna Synthesis Kit (Clontech Laboratories, Inc., Mountain View, California). For quality control and to check for genomic DNA contamination, water blanks and reactions without reverse transcriptase (-RT) were run alongside the normal endometrial RNA samples. On each 96-well plate, water blanks and -RT samples were run in triplicates with each real-time quantitative PCR (Qpcr) reaction.

All primer sets (Table 1) were validated using SYBR ${ }^{\mathrm{TM}}$ Green reagents according to the manufacturer's instructions prior to use with the endometrial explant Cdna samples. Briefly, normal pregnant and nonpregnant endometrial Cdna pools were run in triplicates at a consistent concentration (1ng per reaction) while the forward and reverse primers were run in a checkerboard fashion with working concentrations ranging from $100 \mathrm{Nm}$ to $900 \mathrm{Nm}$. To assess potential DNA contamination and primer dimerization, water blanks were also run in parallel with each primer set combination. The 
optimal primer concentrations were ultimately picked based on the endometrial sample with the lowest Ct value and with the highest $\mathrm{Ct}$ value in the corresponding water blank.

\section{Evaluation of transcript abundance}

Gene expression analysis was performed by using real-time quantitative PCR with the SYBR ${ }^{\mathrm{TM}}$ Green detection assay. Specific primer sequences, the size of the PCR products, and their assay concentrations are shown in Table 1. Peptidylprolyl isomerase A (PPIA) was selected as the bovine reference gene for normalization (Y. Liu et al. 2015; Puech et al. 2015; Walker et al. 2009). All reactions were performed using 1.25ng of Cdna, 6.25UI of 2x SYBR Green PCR Master Mix (Applied Biosystems), and primers at optimized concentrations. Final reaction volumes were made up to $12.5 \mathrm{Ul}$ with Rnase/Dnase-free $\mathrm{H}_{2} \mathrm{O}$. All reactions were carried out in triplicate in a QuantStudio 3 Real-Time PCR System (Applied Biosystems) under the following cycling conditions: $95^{\circ} \mathrm{C}$ for $10 \mathrm{~min} ; 40 \mathrm{cycles}$ of $95^{\circ} \mathrm{C}$ for 15 seconds and $58^{\circ} \mathrm{C}$ for 1 minute. A dissociation curve was included in each Qpcr run to ensure specificity of the amplicons.

\section{Histology}

Fixed endometrial biopsies from each collection timepoint were dehydrated, embedded in paraffin, sectioned into $5 \mu \mathrm{m}$ thick sections and mounted on charged glass microscope slides. All antibodies and antigen retrieval methods used for immunohistochemical staining were previously optimized to work in bovine endometrial tissue.

A general hematoxylin and eosin staining procedure was performed. Briefly, mounted sections were deparaffinized in 3 changes of xylene for 5 minutes each. Sections were then gradually rehydrated 
with decreasing concentrations of ethanol starting at $100 \%$ ethanol and ending in distilled water. Slides were immersed in Meyer Modified Hematoxylin for 6 minutes then washed in distilled water for 2 minutes. Slides were quickly rinsed in $95 \%$ ethanol then immersed in eosin $Y$ solution (1\% eosin $Y, 76 \%$ ethanol) for 30 seconds or up to 1 minute. Sections were then dehydrated with 2 changes of $95 \%$ ethanol for 3 minutes each then 100\% ethanol for 3 minutes. Sections were cleared with 2 changes of xylene for 3 minutes each then set out to air dry completely before mounting with Permount. Permount was allowed to set overnight at room temperature. All slides were examined under a light microscope

\section{Statistical Analysis}

Data were analyzed for homogeneity of variance by single factor ANOVA. Heterogeneity of variance was detected. Where homogeneity of variance was confirmed, a two sample $t$-test assuming equal variances with a two-tail distribution was used to analyze the log-transformed data. Where heterogeneity of variance was found, a two sample $t$-test assuming unequal variance with a two-tail distribution was used to analyze the log-transformed data. The graphs depict normalized, relative expression with accompanying standard errors. Significant differences were displayed as $\mathrm{P}<0.05, \mathrm{P}<0.01$, and $\mathrm{P}<0.001$. 
TABLE 1. List of oligonucleotide sequences, their product sizes, and concentrations used for RT-Qpcr

Oligonucleotide sequences $5^{\prime}$ to $\mathbf{3}^{\prime}$

\begin{tabular}{|c|c|c|c|c|c|c|}
\hline Gene & $\begin{array}{c}\text { Accession } \\
\text { number }\end{array}$ & Forward Primer & $\begin{array}{c}\text { Forward Primer } \\
\text { Concentration }\end{array}$ & Reverse Primer & $\begin{array}{l}\text { Reverse Primer } \\
\text { Concentration }\end{array}$ & $\begin{array}{l}\text { Product } \\
\text { Size (bp) }\end{array}$ \\
\hline CCL2 & NM_174006.2 & CAG CCA GAT GCA ATT AAC TCC CA & $300 \mathrm{Mm}$ & TGC TGG TGA CTC TTC TGT AGT TCA & $300 \mathrm{Mm}$ & 99 \\
\hline CCL8 & NM_174007.1 & GTG CTC GCT CAG CCA GAT TC & $300 \mathrm{Mm}$ & GCT GTC CAG CTT CTT GAA GGG & $100 \mathrm{Mm}$ & 89 \\
\hline CCL11 & NM_205773.2 & ACA TGA AGG TCT CTG CAG TGC T & $900 \mathrm{Mm}$ & TGG TTG GAA TAG AAG CTG GCT GA & $300 \mathrm{Mm}$ & 92 \\
\hline CCL16 & XM_024980640.1 & CCG GCA ATC GTC TTC ATC ACC & $300 \mathrm{Mm}$ & GCG GAT GAA GTC TGG GAT CCT T & $300 \mathrm{Mm}$ & 99 \\
\hline CXCL1 & NM_175700.2 & AGA TGC TAA ACA AGG CTA GTG CC & $300 \mathrm{Mm}$ & СTT TTA СTT САC TTC CAC TGA GGC T & $300 \mathrm{Mm}$ & 89 \\
\hline CXCL2 & NM_174299.3 & GAT GCT AAA CTA GGC CAG CTC TAA & $300 \mathrm{Mm}$ & TTT CTG TAG GGG CAG GGT CT & $100 \mathrm{Mm}$ & 89 \\
\hline CXCL10 & NM_001046551.2 & AGT ACC TTC AGT TGC AGC ACC A & $100 \mathrm{Mm}$ & AGA GAG AGG TAC ACC TTG ACT CAG A & $300 \mathrm{Mm}$ & 89 \\
\hline CXCL12 & NM_001113174.1 & TTC TTT GAG AGC CAT GTC GCC & $300 \mathrm{Mm}$ & CAG CCT TGC CAC GAT CTG AA & $300 \mathrm{Mm}$ & 89 \\
\hline CXCL14 & NM_001034410.2 & GAA CGA GAA GCG CAG GGT CT & $300 \mathrm{Mm}$ & CAA AGT CCT TTG CTT GTT TCC CAA C & $100 \mathrm{Mm}$ & 89 \\
\hline CXCR2 & NM_001101285.1 & AAG CCC AGA ATC ATG GCT GAA A & $100 \mathrm{Mm}$ & TGT AAT TGC CAA AAT CTT CAT CGC T & $300 \mathrm{Mm}$ & 90 \\
\hline MMP1 & NM_174112.1 & ACA GGG ATG AGG TCC GGT T & $300 \mathrm{Mm}$ & TTC ACC GTT CTC GGA AAG CC & $300 \mathrm{Mm}$ & 117 \\
\hline MMP3 & NM_001206637.1 & AGT TCC TGT ACG GGT CTC CC & $300 \mathrm{Mm}$ & CTG CAT CGA AGG ACA AGG CA & $300 \mathrm{Mm}$ & 116 \\
\hline MMP7 & NM_001075130.1 & GGA GCG AAG CAA TCC CAC TG & $300 \mathrm{Mm}$ & GGC CCA TCA AAG GGA TAT GGG & $300 \mathrm{Mm}$ & 108 \\
\hline MMP13 & NM_174389.2 & AAG ACA GAT TCT TCT GGC GGC & $300 \mathrm{Mm}$ & AGG CGG CAT CAA TAC GGT TG & $300 \mathrm{Mm}$ & 101 \\
\hline MMP14 & NM_174390.2 & AGA CAC CAT GAA GGC CAT GAG & $900 \mathrm{Mm}$ & ATG CTG CCA TTT GAG TCC CTG & $900 \mathrm{Mm}$ & 117 \\
\hline PLA2 & NM_174646.3 & ACC TGC AGC AGC GAA AAC AA & $300 \mathrm{Mm}$ & TGT GCT CCT TGT TGT ATG GCA C & $900 \mathrm{Mm}$ & 99 \\
\hline PPIA & NM_178320.2 & GCA TAC AGG TCC TGG CAT CT & $300 \mathrm{Mm}$ & CAC GTG CTT GCC ATC CAA C & $300 \mathrm{Mm}$ & 108 \\
\hline PTGES & NM_174443.2 & CAA AAT GTA CGT GGT GGC CGT & $900 \mathrm{Mm}$ & GCC TCC ATG TCT CTG AGC GT & $300 \mathrm{Mm}$ & 90 \\
\hline PTGS1 & NM_001105323.1 & GCC CGC GCC AGT GAA C & $300 \mathrm{Mm}$ & GTG CGG GTG CAG TCA CAT TG & $300 \mathrm{Mm}$ & 98 \\
\hline PTGS2 & NM_174445.2 & CCC ATG GGT GTG AAA GGG AGG & $300 \mathrm{Mm}$ & GCC CTG GGG ATC AGG AAT GAA & $300 \mathrm{Mm}$ & 95 \\
\hline Regakine & NM_001034220.2 & TCC TCG GTA ACA AGG AAA ATC CCA & $300 \mathrm{Mm}$ & CTG GTC TGG AAG ATG ACC GCT & $300 \mathrm{Mm}$ & 94 \\
\hline
\end{tabular}




\section{RESULTS}

\section{Changes in transcript abundance of endometrial chemokines}

Endometrial explants from nonpregnant and pregnant heifers were incubated for 48 and 72 hours in the presence or absence of $15 \mu \mathrm{g} / \mathrm{ml}$ bovine PAGs (boPAG) in the culture medium. The PAGs used in this culture system were the same as those used by Wallace and others (Wallace et al 2019). Briefly, the boPAG isolate consisted primarily of boPAG4, boPAG6, and boPAG9, which are all modern PAGs that are produced by the BNCs. To further explore the effect of the PAGs on chemokines in the bovine endometrium, we analyzed the collected explants for various chemokine transcripts using RTQpcr. The ability of this PAG preparation to alter transcript abundance was evaluated for 11 chemokine transcripts (Table 1). The abundance of each was determined relative to peptidylprolyl isomerase A (PPIA). The results are presented by status (pregnant or nonpregnant) with the results of the nontreated control samples next to those of the PAG-treated samples.

In the nonpregnant samples, transcript abundance of chemokines CXCL1 $(P<0.01), C X C L 2$ $(P<0.01)$ and $C X C L 5(P<0.001)$ were all significantly increased in response to boPAG after 48 and $72 \mathrm{hr}$ (Figure 6A). Conversely, transcripts for CCL2 $(\mathrm{P}<0.05)$, CCL8 $(\mathrm{P}<0.001)$ and CCL16 $(\mathrm{P}<0.05)$ were decreased in response to boPAG at each timepoint. CXCL14 and CCL11 were increased in the nonpregnant samples only after $72 \mathrm{hr}(\mathrm{P}<0.05)$ of culture with boPAG (Figure 6A and 6B). Some chemokines were not affected by treatment in the nonpregnant samples; CXCL10, CXCL12 and Regakine were not significantly changed by treatment, although there was an overall trend toward a decrease in transcript abundance of these transcripts (Figure 6C).

In the pregnant samples, transcripts for CXCL1, CXCL2 and CXCL5 were significantly increased at both the 48 and $72 \mathrm{hr}$ timepoints $(\mathrm{P}<0.001$; Figure $7 \mathrm{~A})$. Much like in the nonpregnant samples, CCL2 $(P<0.05)$ and $C C L 8(P<0.001)$ were decreased in response to boPAG at both timepoints. Unlike the 
nonpregnant samples, where CCL11 was increased only at $72 \mathrm{hr}, C C L 11$ was increased at both timepoints in the pregnant samples $(P<0.05) . C X C L 14$ was decreased in response to boPAG in the pregnant samples but it was only significant at the 72hr timepoint (Figure 7A and 7B). An effect of status was seen for CXCL10 $(\mathrm{P}<0.001)$, CXCL12 $(\mathrm{P}<0.01)$, and Regakine $(\mathrm{P}<0.01)$. All three transcripts were significantly decreased in response to boPAG in the pregnant samples but not the nonpregnant (Figure 7C).

To better illustrate the individual responses generated by treatment of bovine endometrium with boPAG, we also presented the data as a ratio of relative transcript abundance of the boPAG-treated explants vs. the respective non-treated controls. This was done for each transcript analyzed and the data are represented in Figures 9 and 10. These data also allowed us to more clearly see common trends of changes in transcript abundance between the nonpregnant and the pregnant samples. In both the nonpregnant (Figure 8) and pregnant (Figure 9) endometrial samples, CXCL1, CXCL2 and CXCL5 were all greatly increased by treatment with boPAG after 48 and $72 \mathrm{hr}$ while CCL2, CCL8 and CCL16 were all decreased by treatment with boPAG after 48 and $72 \mathrm{hr}$ in culture. CCL11 was also increased by boPAG in both nonpregnant and pregnant samples but the fold-change was greater in the pregnant samples. While the overall trend of decreasing transcript abundance of CXCL10, CXCL12, and Regakine in response to boPAG was present in both pregnant and nonpregnant groups, the fold-change was much greater in the pregnant samples. The one difference between the pregnant and nonpregnant groups was the change in CXCL14. CXCL14 was increased by boPAG in the nonpregnant samples at $72 \mathrm{hr}$ $(P<0.05)$ but was decreased in the pregnant samples at both the 48 and $72 \mathrm{hr}$ time points $(P<0.05)$.

\section{Histology}

To evaluate the effects of boPAG on a whole tissue level we fixed some of the collected endometrial explants after $24 \mathrm{hr}, 48 \mathrm{hr}, 72 \mathrm{hr}$ and $96 \mathrm{hr}$ of culture with or without added boPAG for 
histological analysis. The histological effects of boPAG were similar between the pregnant and nonpregnant groups so the results stated here will be generalized for both groups. Treatment with boPAG seemed to have an effect on glandular morphology. After $24 \mathrm{hr}$ in culture, many of the endometrial glands has areas of very thin or missing epithelium. In the lumen of those glands was darkly stained cellular material. Some glands lacked epithelia entirely. The luminal epithelium also took on an irregular morphology in the boPAG treated tissues. There were areas where the luminal epithelium was very thin or even missing and in some cases the epithelium appeared vacuolated. In some cases the stroma appeared more diffuse than its untreated counterpart. The untreated control tissue after $24 \mathrm{hr}$ of culture showed some signs of glandular epithelial sloughing, as seen by darkly stained cells in the lumen, but the overall morphology of the glandular and luminal epithelia were normal (Figures 10A and 10B). By 48h, many of the boPAG-treated endometrial glands had entirely lost their epithelium. The luminal epithelium was still irregular with visible areas of detachment (Figures 10C and 10D). After $72 \mathrm{hr}$ in culture, it appeared that the some of the glandular epithelia as well as the luminal epithelia in the boPAG treated tissues were starting to grow back. The untreated controls at this time were starting to exhibit areas of thin or missing epithelium (Figures 10E and 10F). By 96hr in culture, the PAG-treated tissues were beginning to look much more normal. In the boPAG-treated tissues the glandular and luminal epithelia were returning to normal and the amount of cellular debris in the glandular lumen was decreased, though the luminal epithelium was still irregular. The untreated controls were now showing signs of glandular cell death and an irregular luminal epithelium (Figures $10 \mathrm{G}$ and $10 \mathrm{H})$. 

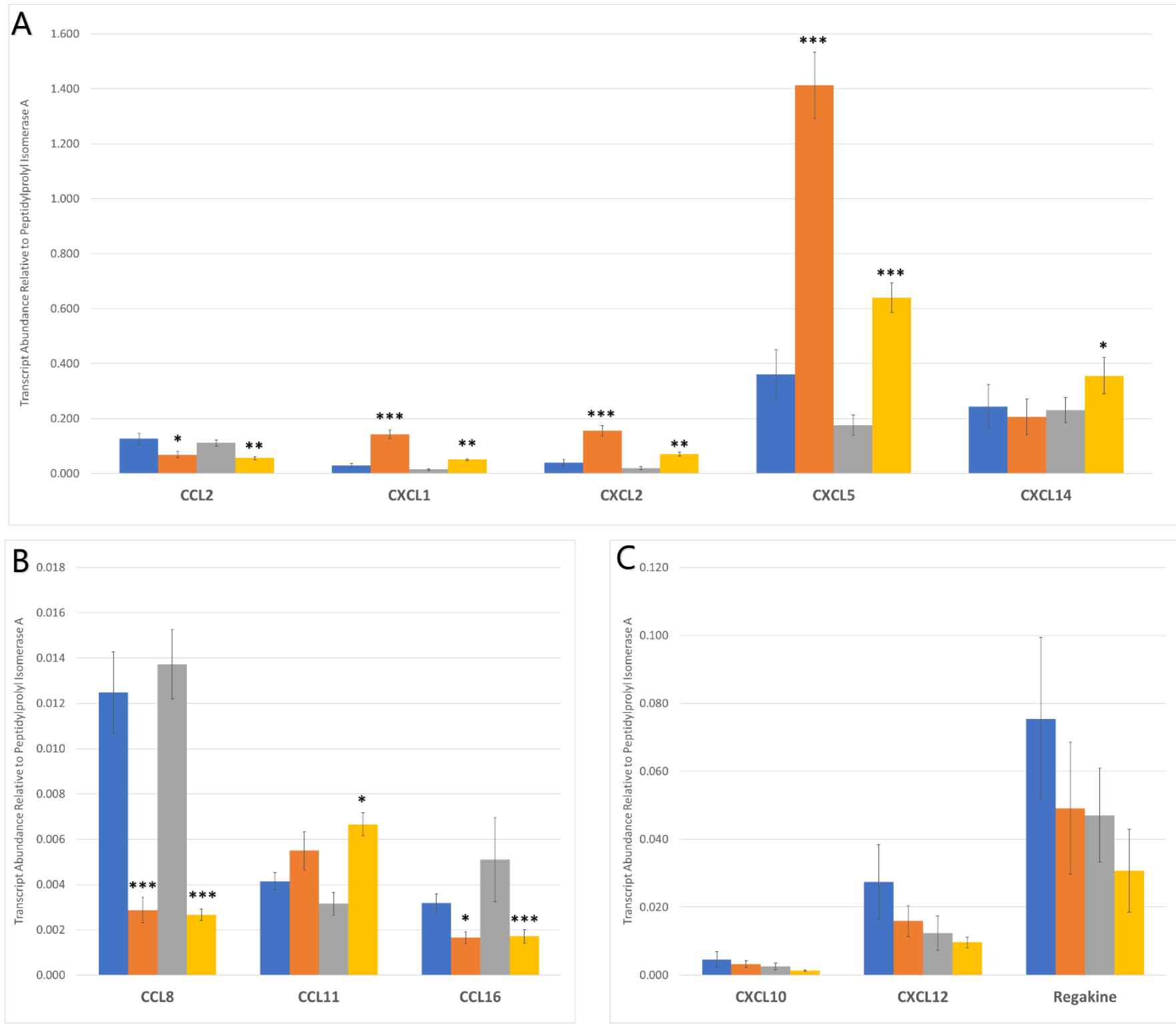

Figure 6. Relative transcript abundance of chemokines in nonpregnant endometrium after exposure to PAGs for 48 and $72 \mathrm{hr}$

Target transcript abundance of several chemokines in endometrial explants from nonpregnant heifers exposed to PAGs for 48 (blue and orange bars) and 72 hours (grey and yellow bars). Data was quantified by RT-PCR and the results were normalized to PPIA. Data are presented as mean \pm SEM. Blue bar: $48 \mathrm{hr}$ nontreated endometrium; Orange bar: 48hr PAG-treated endometrium; Grey bar: $72 \mathrm{hr}$ nontreated endometrium; Yellow bar: 72hr PAG-treated endometrium. ${ }^{*} \mathrm{P}<0.05 ;{ }^{*} \mathrm{P}<0.01 ;{ }^{* *} \mathrm{P}<0.001$. 
A

A 1.600

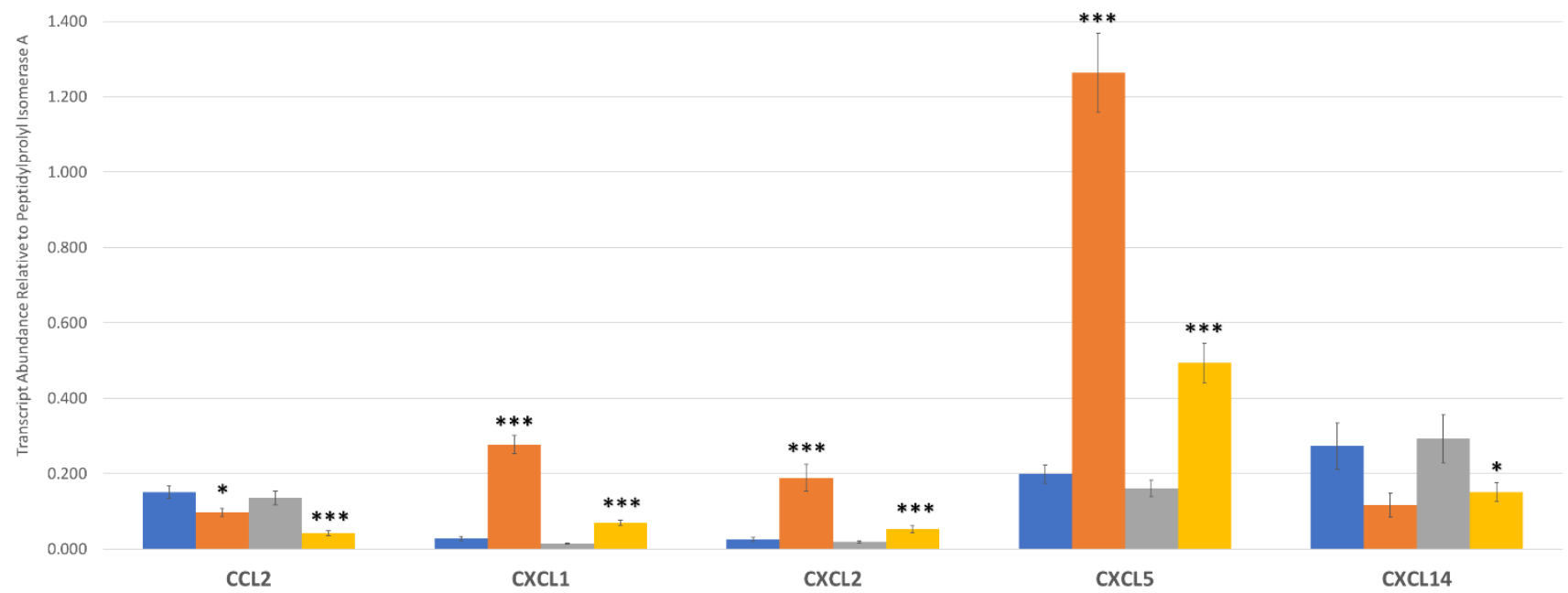

B

$\mathrm{B}_{0.070}$

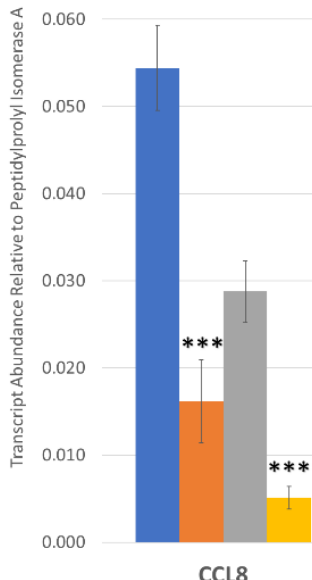

CCL8

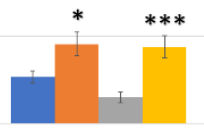

CCL11

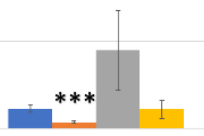

CCL16
$C_{0.080}$

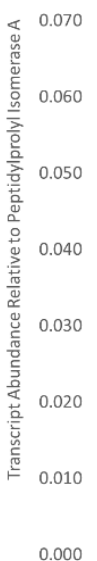

0.000

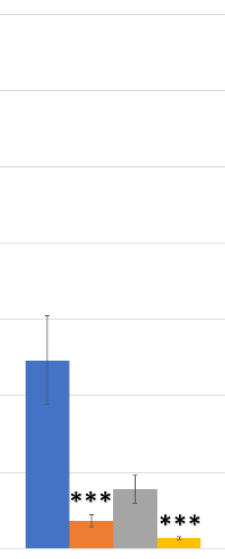

CXCL10

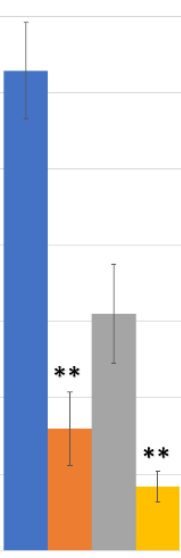

CXCL12

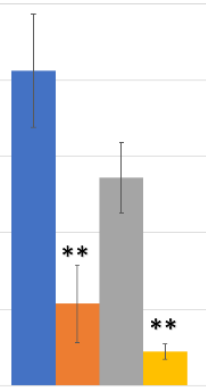

Regakine

Figure 7. Relative transcript abundance of chemokines in pregnant endometrium after exposure to PAGs for 48 and $72 \mathrm{hr}$

Target transcript abundance of several chemokines in endometrial explants from pregnant heifers exposed to PAGs for 48 (blue and orange bars) and 72 hours (grey and yellow bars). Data was quantified by RT-PCR and the results were normalized to PPIA. Data are presented as mean \pm SEM. Blue bar: $48 \mathrm{hr}$ nontreated endometrium; Orange bar: 48hr PAG-treated endometrium; Grey bar: 72hr nontreated endometrium; Yellow bar: 72hr PAGtreated endometrium. ${ }^{*} \mathrm{P}<0.05 ;{ }^{*} \mathrm{P}<0.01 ;{ }^{* *} \mathrm{P}<0.001$. 


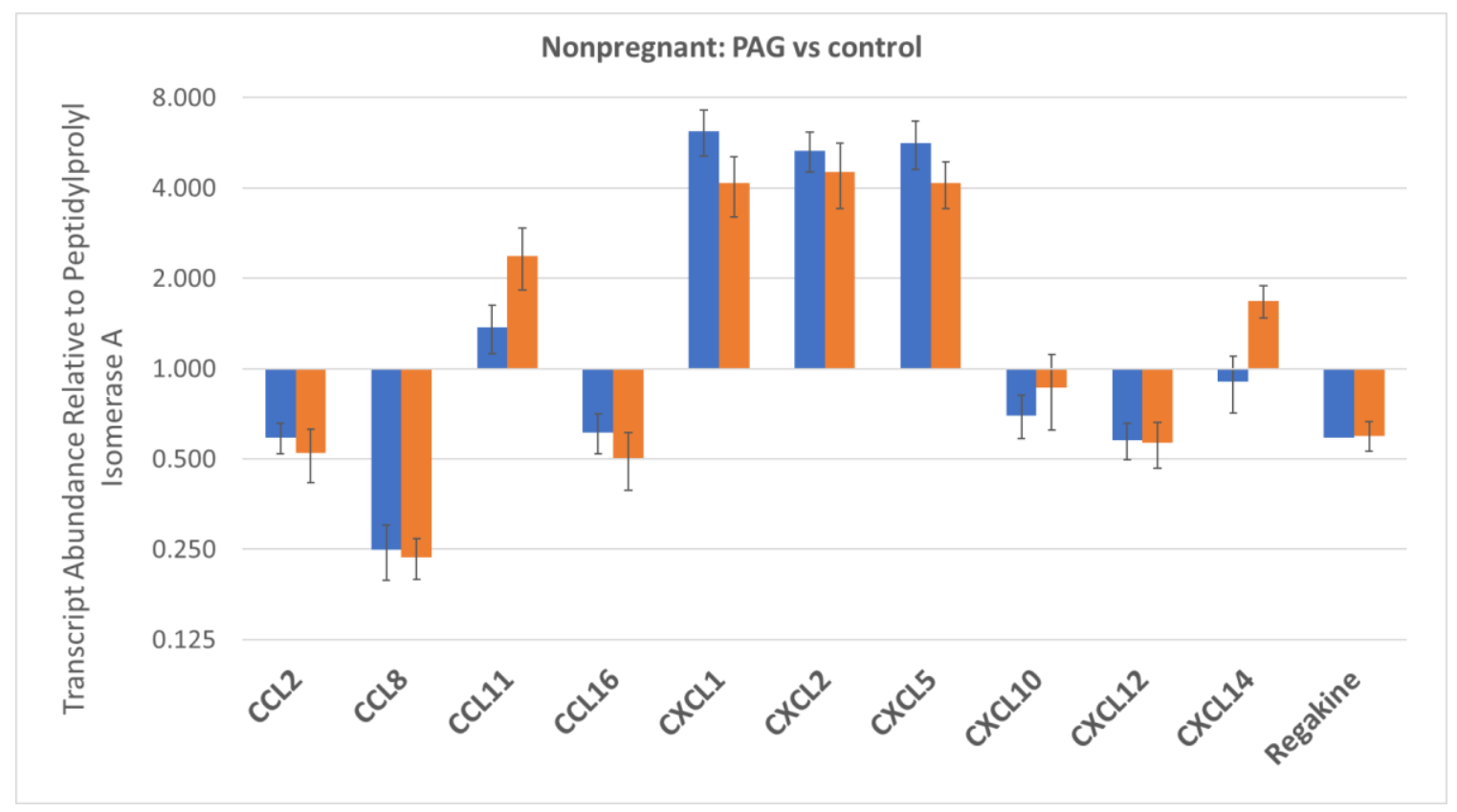

Figure 8. PAG-treated vs Nontreated transcript abundance of chemokines in nonpregnant endometrium after exposure to PAGs for 48 and $72 \mathrm{hr}$

Target transcript abundance of several chemokines in endometrial explants from nonpregnant heifers exposed to PAGs for 48 (blue bars) and 72 hours (orange bars). Data are represented as a ratio of PAG-treated:Nontreated relative abundance Data was quantified by RT-PCR and the results were normalized to PPIA. Data are presented as mean \pm SEM. Blue bar: $48 \mathrm{hr}$ PAG-treated vs nontreated control endometrium; Orange bar: $72 \mathrm{hr}$ PAG-treated vs nontreated control endometrium 


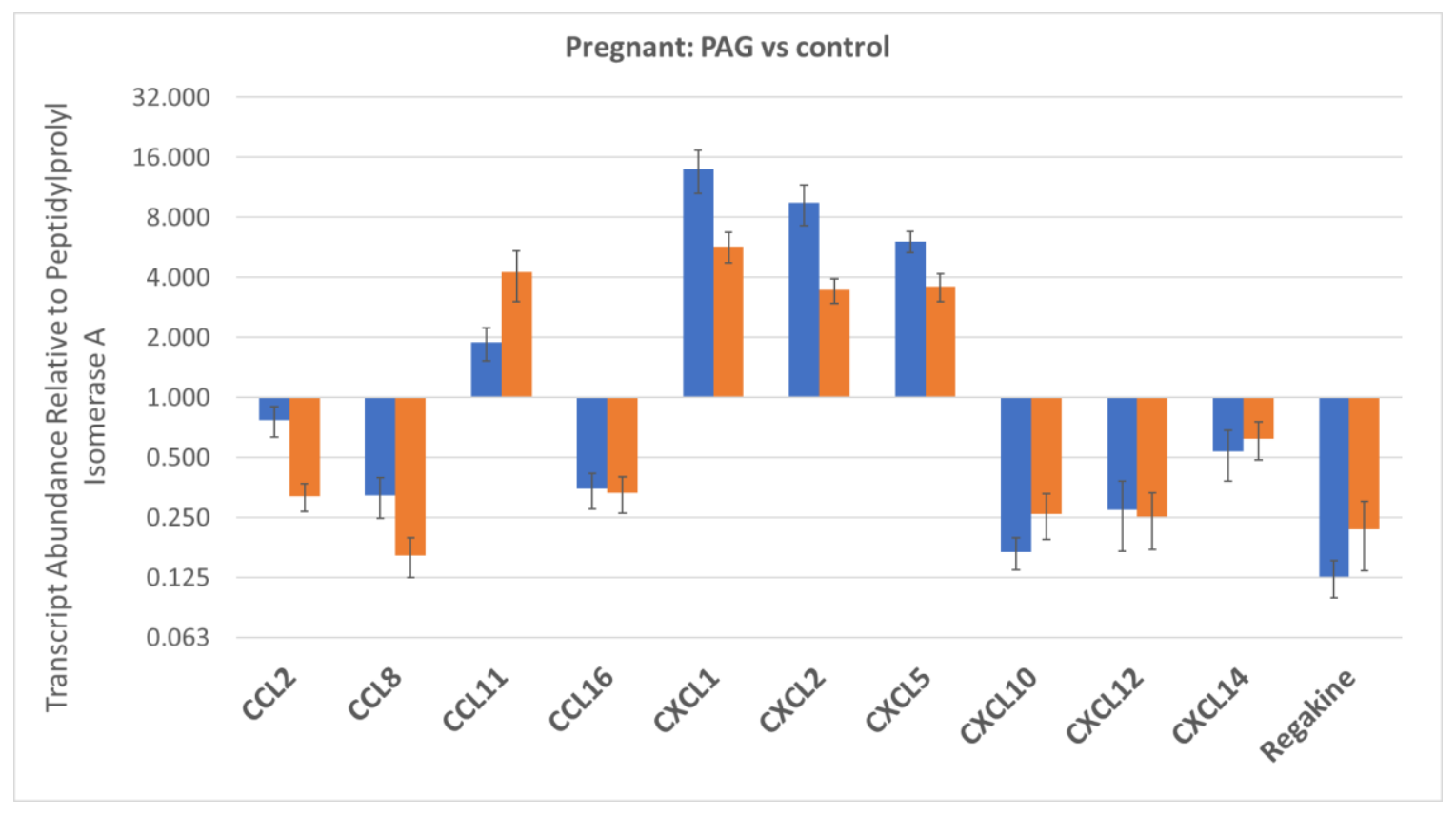

Figure 9. PAG-treated vs Nontreated transcript abundance of chemokines in pregnant endometrium after exposure to PAGs for 48 and $72 \mathrm{hr}$

Target transcript abundance of several chemokines in endometrial explants from pregnant heifers exposed to PAGs for 48 (blue bars) and 72 hours (orange bars). Data are represented as a ratio of PAG-treated:Nontreated relative abundance Data was quantified by RT-PCR and the results were normalized to PPIA. Data are presented as mean \pm SEM. Blue bar: $48 \mathrm{hr}$ PAG-treated vs nontreated control endometrium; Orange bar: $72 \mathrm{hr}$ PAG-treated vs nontreated control endometrium 
Control

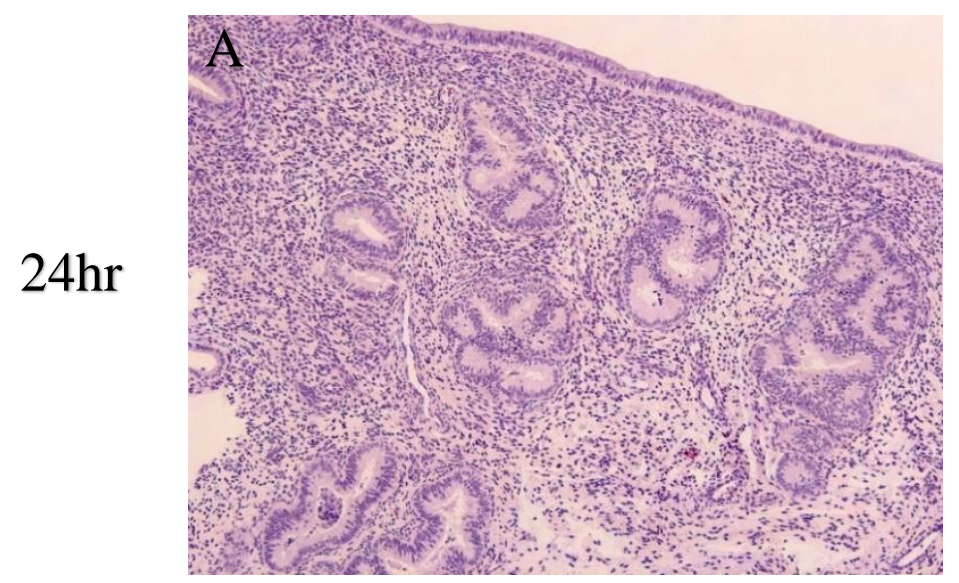

48hr

$72 \mathrm{hr}$

\section{C.}
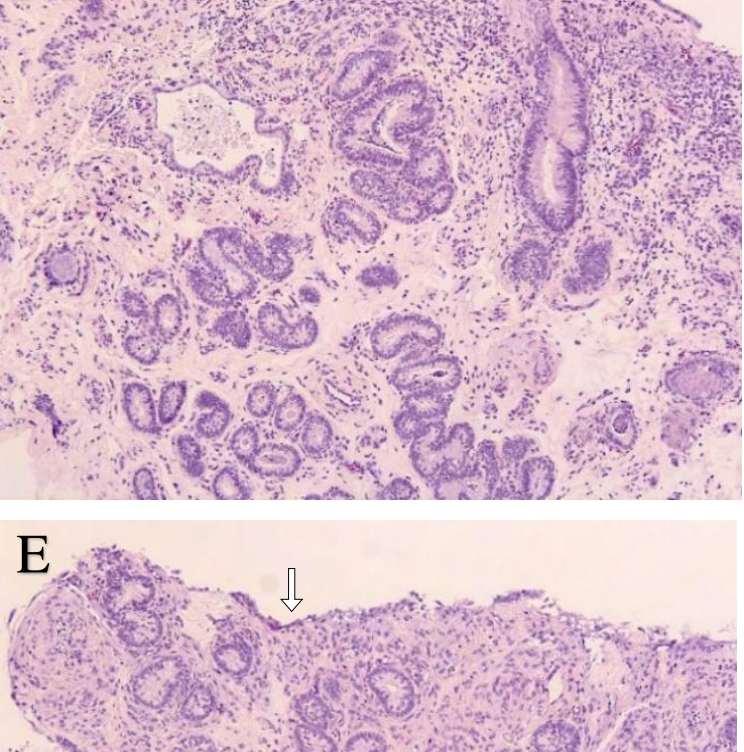
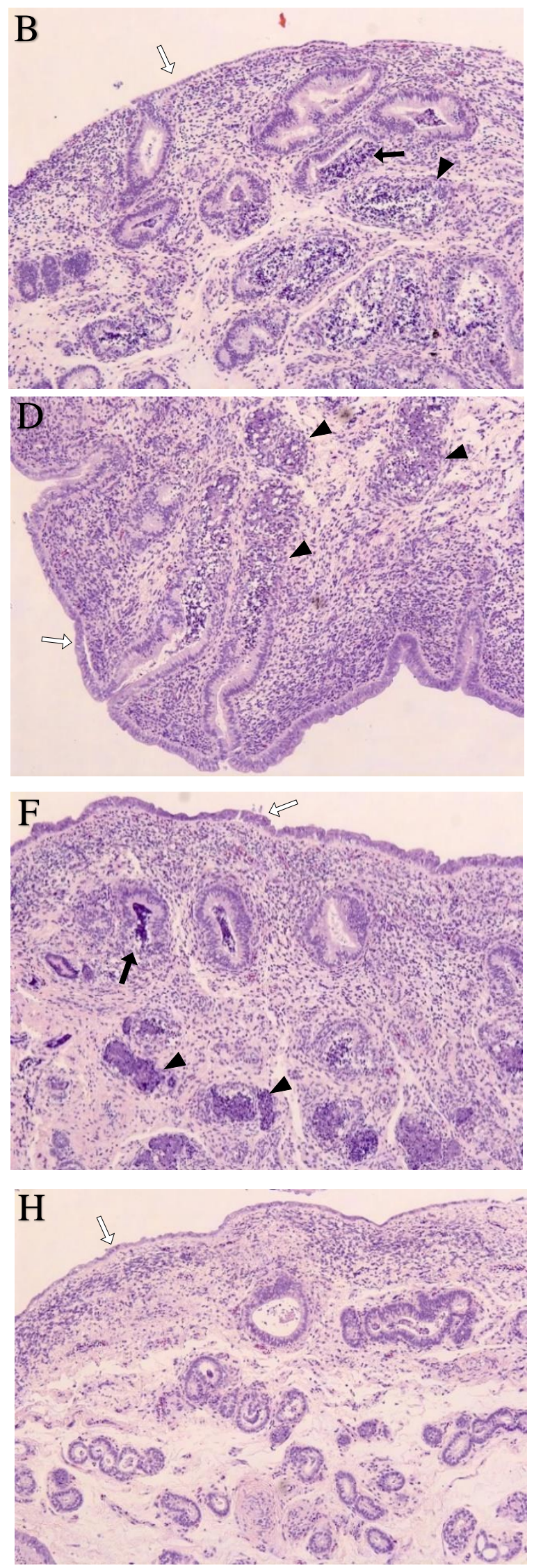
Figure 10. H\&E staining of bovine endometrial explants after exposure to PAGs for 24,48 , 72, and 96 hours

Representative $H \& E$ staining of bovine endometrial explants cultured without $(A, C, E, G)$ or with $(B, D, F, H)$ bovine PAGs. Results were similar between nonpregnant and pregnant samples so a single representative is shown here. Samples in culture for $24 \mathrm{hr}(A$ and B); Samples cultured for $48 \mathrm{hr}$ (C and D); Samples cultured for $72 \mathrm{hr}$ ( $\mathrm{E}$ and $\mathrm{F}$ ); Samples cultured for $96 \mathrm{~h}$ ( $\mathrm{G}$ and $\mathrm{H}$ ); Areas of irregular luminal epithelium were denoted with a white arrow $(\Rightarrow)$; Areas of irregular glandular epithelium were denoted with a black arrow $(\rightarrow)$; Areas of missing glandular epithelium were denoted with arrowheads $(-$ ).

\section{DISCUSSION}

The ruminant PAGs have been a subject of study since the early 1980's. Much of the published literature on the PAGs to date has focused on characterizing these proteins as tools for pregnancy diagnosis and pregnancy maintenance in ruminants (Green et al, 2005; Pohler et al, 2013 and 2016; Wallace et al, 2015; Xie et al, 1997b; Zoli et al, 1992). Additional research has been conducted to characterize the spatial and temporal expression patterns of ruminant PAGs (Green et al, 2000; Patel et al, 2004; Touzard et al, 2013; Wooding et al, 2005; Xie et al, 1997b). Some possible functions for the PAGs have been proposed, but even after years of study there is still little understood about how they are functioning during pregnancy. A suggested role for the PAGs is that they could be serving an immunomodulatory role during pregnancy (Austin et al, 1999; Dosogne et al, 1999; Hoeben et al, 1999 and 2000; Klisch et al, 2006; Mathialagan and Hansen, 1996; Serrano-Pérez et al, 2016; Wallace et al, 2015). Though mostly speculative, there is some evidence to support this theory. Recently, our lab demonstrated that bovine PAGs, when used to treat bovine endometrial explants for 24 and 96 hours, can alter expression of the endometrial chemokine CXCL5 (Wallace et al, 2019). Other research by Austin et al. (1999) showed similar results when endometrial explants and endometrial cells were treated with PSPB (a protein now known to be identical or similar to boPAG1). Whether this is a direct or indirect action of the PAGs remains unclear. However, what is clear is that the PAGs are, at least in part, likely performing their functional roles at the placenta-uterine interface. This led us to evaluate 
this possibility further. The PAGs investigated in these experiments (a mixture consisting predominantly of boPAG4, -6 and -9 ) were ones produced by giant binucleated trophoblasts. Day 18 represented a stage in which the endometrium was likely capable of responding to these PAGs. Additionally, the tissue would not have been exposed to the particular PAGs used in these experiments prior to the in vitro culture because they are not expressed by the trophoblasts until after day 18 (R. M. Wallace et al. 2019).

Immune cells are present in the bovine endometrium throughout the estrous cycle and throughout pregnancy (Leung et al. 2000; Tekin and Hansen 2004; Velázquez et al. 2019). During pregnancy, immune regulation in the ruminant endometrium is important for fetal survival. Changes in the uterine immune cell population as well as immune cell function during pregnancy is driven by local signals from the conceptus and from hormonal changes that are mediated by either the placenta or the maternal system. Interferon tau (IFNT), a type 1 interferon secreted by the placental trophoblasts between days 15 and 21 of bovine pregnancy (Fuller W. Bazer et al. 2008; Ealy and Yang 2009; Ealy and Wooldridge 2017), is one such signal from the conceptus that is thought to be responsible for altering uterine immune cell function to create an environment conducive for embryonic growth and development (Gifford et al. 2007a; Glaucia Teixeira et al. 1997; Rashid et al. 2018; Talukder et al. 2017). Not only can IFNT stimulate classical interferon-stimulated genes in both the endometrium (M.-S. Kim, Min, and Imakawa 2013) and circulating immune cells (Gifford et al. 2007a), but it can also stimulate the release of interferon-inducible chemokines, such as CXCL10, in the endometrium (Imakawa et al. 2006). Progesterone is a key regulator of pregnancy and also uterine immune function (Lonergan and Forde 2015; Lonergan, Forde, and Spencer 2016; P. J Hansen 1998). Progesterone can inhibit proliferation of activated peripheral blood lymphocytes in vitro and reduce the number of uterine CD45R+ lymphocytes in vivo in sheep (Staples et al. 1983; Staples et al. 1984; Monterroso and Hansen 1993; Segerson et al. 1997). In sheep, progesterone has been shown to induce the release of a serine protease inhibitor known as uterine serpin, which has been shown to inhibit lymphocyte proliferation in vitro in sheep 
(Peltier, Liu, and Hansen 2000) and natural-killer cell-mediated abortion in mice (W.-J. Liu and Hansen

1993). Both IFNt and progesterone are thought to have either direct or indirect effects on uterine immune functions during early pregnancy, which is crucial for proper implantation and embryo development.

Chemokines are a family of small 8-10kDa cytokines noted for their roles in immune cell chemotaxis into sites of inflammation. They can be separated into four main groups based on the number and spacing of conserved $\mathrm{N}$-terminal cysteine residues; $\mathrm{C}, \mathrm{CC}, \mathrm{CXC}$, and $\mathrm{CX}_{3} \mathrm{C}$. The $\mathrm{CXC}$ chemokine family can be further subdivided into 2 groups based on the presence (ELR+) or absence (ELR-) of an 'ELR motif' (amino acid sequence Glu-Leu-Arg) immediately preceding the first N-terminal cysteine (Balestrieri et al. 2008). The ELR+CXC chemokines are known as the neutrophil activating chemokines (NACs) because of their abilities to specifically recruit neutrophils into sites of inflammation (Rajarathnam et al. 2019). In cattle, the ELR+ CXC chemokines consist of CXCL1, CXCL2, CXCL3, CXCL5, CXCL7 and CXCL8/IL-8. The findings of the present study demonstrate that bovine PAGs are capable of upregulating endometrial expression of several NACs: CXCL1, CXCL2, and CXCL5. This may suggest a role for the PAGs in immune cell recruitment. Currently, endometrial neutrophil infiltration in early pregnancy is not well characterized in cattle. However, several studies have documented the deleterious effects of endometrial neutrophils in endometritis on reproductive performance (Lincke et al. 2007; LeBlanc et al. 2002; Gilbert et al. 2005; Kim and Kang 2003). Because the PAGs are such an abundant product of the placenta throughout pregnancy, it is unlikely that the PAGs would promote neutrophil recruitment for the purpose of potentially damaging inflammation. There is evidence of different subgroups of neutrophils that possess different functional qualities based on cytokine production, ability to activate macrophages, cell surface receptor expression, and cell surface antigen expression (Tsuda et al. 2004; Fridlender et al. 2009; Pillay et al. 2010). Perhaps these chemokines could be recruiting/activating neutrophils of a less inflammatory phenotype, or perhaps these chemokines are 
not recruiting neutrophils at all and, rather, are performing some local function in the endometrium. This is speculation, of course. More research will need to be conducted to further explore the potential roles of these chemokines in the bovine endometrium.

Along with $C X C L 1, C X C L 2$, and $C X C L 5$ being upregulated, PAGs also induced an increase in CCL11 expression. CCL11 is known to recruit eosinophils to sites of inflammation (Gouon-Evans and Pollard 2001). Together, all four of these chemokines recruit granulocytes. Little is known about the role of CCL11 in the reproductive tract. However, it's receptor CCR3 is present on the endometrial luminal epithelium, glandular epithelium, and on the trophectoderm (Sakumoto et al. 2017), so it is possible that CCL11 is functioning locally in the endometrium or at the interface. CCL11 has be shown to regulate extravillous trophoblast migration, invasion, and adhesion in human pregnancy (Chau et al. 2013). Perhaps CCL11 could be acting locally at the utero-placental interface to regulate conceptus attachment via its receptor, CCR3, on both epithelial surfaces.

CCL2, CCL8, CCL16, CXCL10, CXCL12, and CXCL14 are all chemotactic for monocytes and/or macrophages. In particular, CCL2, CCL8, and CXCL10 have been shown to be present in the ovine endometrium during pregnancy and upregulated by IFNT (Asselin et al. 2001; Andoh et al. 2020; Nagaoka, Sakai, et al. 2003). It is interesting here that we would see the opposite response after treatment with PAGs in bovine tissue. However, expression levels of $C X C L 10$ were increased in the nontreated pregnant endometrium compared to the non-treated nonpregnant endometrium. This is in agreement with previous data that CXCL10 is upregulated in early pregnancy due to IFN stimulation (Nagaoka, Sakai, et al. 2003). Additionally, early bovine pregnancy is characterized by an expansion of endometrial macrophages and dendritic cells (Mansouri-Attia et al. 2012; Oliveira et al. 2010). Upregulation of these chemokines would fit that narrative as they all have macrophage chemoattractant properties. Perhaps the negative regulatory effect of the PAGs is normally canceled out in vivo due to the positive effects of IFNt. However, individual functions of the PAGs cannot be ruled out. The 
particular PAGs that were used in these experiments are not normally expressed this early in pregnancy. It is possible that the PAGs expressed during conceptus attachment have different individual functions than the later expressed PAGs. Furthermore, CXCL10 and CXCL12 expression was only significantly affected in the pregnant endometrium. Since progesterone levels in the nonpregnant and pregnant groups were made to be similar (R. M. Wallace et al. 2019), this might suggest that regulation of these chemokines by PAGs is dependent on other pregnancy-specific factors, such as IFNt.

Unfortunately, little is known about the roles of CCL16 and CXCL14 in the endometrium. CCL16 has been shown to increase antigen presentation by macrophages and enhance T-cell cytotoxicity during inflammatory reactions (Cappello et al. 2004). All of which would be detrimental to pregnancy establishment. Downregulation of this chemokine would imply an immunoprotection function of the PAGs. CXCL14 is known to play critical roles in upregulation of $\mathrm{MCH}-\mathrm{I}$ molecules on tumor cells as well as establishing immune surveillance in normal epithelial layers (Westrich et al. 2020). This would further support the idea that PAGs could be serving an immunoprotective role by indirectly decreasing the availability of harmful pro-inflammatory cells in early pregnancy.

As previously mentioned, the NACs are known to recruit neutrophils but in humans and mice they also characteristically promote angiogenesis by signaling through the receptor CXCR2 on neutrophils and endothelial cells (Addison et al. 2000; Strieter et al. 2006b). The ELR motif of these chemokines appears to be necessary for angiogenesis. Corneal micropocket assays using recombinant human CXCL1, CXCL5 and CXCL8 induced angiogenesis in rat corneas, without evidence of significant leukocyte infiltration. When the ELR motif is mutated to TVR (corresponding to the sequence of CXCL10, an anti-angiogenic ELR- chemokine) the angiogenic activity of CXCL8 is inhibited (Strieter et al. 1995). Results of the current paper showed that CXCL1, CXCL2, and CXCL5 were all upregulated in the endometrium after exposure to bovine PAGs. This may suggest a role for the PAGs in the initiation of endometrial angiogenesis during early pregnancy. This is an interesting concept because the PAGs have 
never been implicated in the promotion of angiogenesis before now. Furthermore, the PAGs were also able to downregulate expression of CXCL10, a known anti-angiogenic chemokine (Strieter et al. 1995). CCL2 has also been implicating in inhibition of angiogenesis in mice (Bonapace et al. 2014). This would further support the idea that PAGs may be promoting an angiogenic environment in the endometrium through chemokine signaling. Contrastingly, CXCL12 is a known promoter of angiogenesis (Salcedo and Oppenheim 2003; Quinn et al. 2014) and its expression was downregulated by PAGs in the pregnant endometrium. This doesn't quite fit the model of endometrial angiogenesis, however, CXCL12 is also important in the mobilization of monocytes and T-cells from the bone marrow and lymph nodes, respectively (Kryczek et al. 2005; Ding and Morrison 2013). Therefore, this would fit the model of immunoprotection of the attaching conceptus.

Regakine is a recently discovered CC chemokine that is present in high concentrations in bovine plasma and has been shown to synergize with CXCL8 to recruit neutrophils (Struyf et al. 2001; Gouwy et al. 2002). It is a very unique chemokine that shows less than $50 \%$ sequence identity to any known human or animal chemokine (Struyf et al. 2001) so it appears to be ruminant- (if not bovine-) specific (Widdison and Coffey 2011). It's function within the body is still not well characterized so it is difficult to imply its function in the endometrium. It currently has no known receptor so it's exact target cells or tissues are unclear as well. It could be possible that it has no receptor and its main function is to synergize with other chemokines, such as CXCL8, to enhance their chemotactic properties (Gouwy et al. 2004). If this is the case, then the downregulation of Regakine together with the upregulation of CXCL5 (a chemokine also known to synergize with Regakine; Struyf et al. 2001; Gouwy et al. 2002) might imply that CXCL5 and its family members may not be recruiting neutrophils but performing some other local function, such as promotion of angiogenesis.

In summary, these data provide evidence that the binucleate cell-specific bovine PAGs are participating in local immunomodulation of the maternal immune system in the endometrium of cattle. 
These results are supported by previously published data indicating that PAGs can induce expression of certain chemokines (e.g. CXCL5/CXCL6). However, some of these data conflict with our current knowledge of the immune populations in the endometrium of pregnant animals. Whether this is the result of the in vitro culture system or a true result of PAGs function remains to be determined. The prospect of the PAGs serving as immunomodulatory proteins and/or as pro-angiogenic factors is intriguing. Since many binucleate cell derived PAGs are delivered to the maternal endometrial stroma throughout pregnancy, this makes them good candidates for the functions proposed here. Further studies into the role(s) of the PAGs will surely provide more insight into the functions proposed in this paper. 


\section{APPENDIX I}

The use of a monoclonal antibody to rapidly purify bovine pregnancy-associated glycoproteins.

\section{Summary}

Protein purification permits the isolation of target proteins in order to study their function,

structure, and interactions with other proteins; however, purification of proteins, like PAGs, tends to be a laborious process. In the past, recombinant PAGs have been developed and numerous purification schemes have been utilized (S. Xie et al. 1997; Green et al. 2005b; Bériot et al. 2014); however, they consist of several steps and, in both cases, recovery yields are low relative to the amount of starting material. Therefore, a more efficient purification method was developed utilizing 87 mmune-affinity chromatography with an anti-PAG monoclonal antibody.

\section{Materials}

\section{Homogenization of Cotyledonary Extract}

1. Cotyledons from mid-gestational bovine placenta

2. Homogenizer

3. 50,000 molecular weight dialysis tubing

4. Cotyledon Homogenization Buffer: $68.5 \mathrm{Mm} \mathrm{NaCl}, 1.35 \mathrm{Mm} \mathrm{KCl}, 5 \mathrm{Mm} \mathrm{Na} 2 \mathrm{HPO}_{4} * 7 \mathrm{H}_{2} \mathrm{O}$, Ph 7.4

5. Dialysis buffer A: $20 \mathrm{Mm}$ Tris, Ph 8.0

6. Buffer A: $20 \mathrm{Mm}$ Tris, $1.0 \mathrm{M} \mathrm{NaCl}, 1.0 \mathrm{Mm}$ EDTA, $0.2 \mathrm{Mm}$ PMSF, $0.02 \% \mathrm{NaN}_{3}, 0.1 \mathrm{Mm} 2-$ mercaptoethanol, $\mathrm{Ph} 8.0$

7. Buffer B: $20 \mathrm{Mm}$ Tris, $0.8 \mathrm{M} \mathrm{NaCl}, 1.0 \mathrm{Mm}$ EDTA, $0.2 \mathrm{Mm}$ PMSF, $0.02 \% \mathrm{NaN}_{3}, 0.1 \mathrm{Mm} 2-$ mercaptoethanol, $\mathrm{Ph} 7.5$

8. Buffer C: $20 \mathrm{Mm}$ Tris, $0.6 \mathrm{M} \mathrm{NaCl}, 1.0 \mathrm{Mm}$ EDTA, $0.2 \mathrm{Mm}$ PMSF, $0.02 \% \mathrm{NaN}_{3}, 0.1 \mathrm{Mm} 2-$ mercaptoethanol, $\mathrm{Ph} 7.5$

9. Buffer D: $20 \mathrm{Mm}$ Tris, $0.4 \mathrm{M} \mathrm{NaCl}, 1.0 \mathrm{Mm}$ EDTA, $0.2 \mathrm{Mm}$ PMSF, $0.02 \% \mathrm{NaN}_{3}, 0.1 \mathrm{Mm} 2$ mercaptoethanol, $\mathrm{Ph} 7.5$

10. Buffer E: $20 \mathrm{Mm}$ Tris, $150 \mathrm{Mm} \mathrm{NaCl}, 1.0 \mathrm{Mm}$ EDTA, 0.2 Mm PMSF, $0.02 \% \mathrm{NaN}_{3}, 0.1 \mathrm{Mm} 2-$ mercaptoethanol, $\mathrm{Ph} 7.0$

11. Screw top plastic centrifuge tubes

\section{Ammonium Sulfate Precipitation of Cotyledonary Extract}

1. Ammonium Sulfate

2. Screw top plastic centrifuge tubes

3. Large beakers

4. Re-suspension buffer: $137 \mathrm{Mm} \mathrm{NaCl}, 2.7 \mathrm{Mm} \mathrm{KCl}, 10 \mathrm{Mm} \mathrm{Na} 2 \mathrm{HPO}_{4} * 7 \mathrm{H}_{2} \mathrm{O}, \mathrm{Ph} 7.4$

5. 50,000 molecular weight dialysis tubing

6. Dialysis buffer B: $137 \mathrm{Mm} \mathrm{NaCl}, 2.7 \mathrm{Mm} \mathrm{KCl}, 10 \mathrm{Mm} \mathrm{Na} 2 \mathrm{HPO}_{4}{ }^{*} 7 \mathrm{H}_{2} \mathrm{O}$, Ph 7.4

7. Dialysis buffer C: $0.1 \mathrm{M}$ Tris, $0.15 \mathrm{M} \mathrm{NaCL}$, Ph 7.6 


\section{Preparation of Affinity Chromatography Column}

1. EZ-Link NHS-LC-LC biotin (Pierce 21343)

2. Agarose Avidin D (Vector Laboratories A-2010)

3. Anti-PAG monoclonal (L4)

4. Dialysis buffer $B$ (see section 2.2)

5. Equilibration buffer: $137 \mathrm{Mm} \mathrm{NaCl}, 2.7 \mathrm{Mm} \mathrm{KCl}, 10 \mathrm{Mm} \mathrm{Na} 2 \mathrm{HPO}_{4} * 7 \mathrm{H}_{2} \mathrm{O}, \mathrm{Ph} 7.4$

\section{Affinity Chromatography Purification of PAGs}

1. Wash buffer A: $0.1 \mathrm{M}$ Tris, $0.15 \mathrm{M} \mathrm{NaCl}, \mathrm{Ph} 7.6$

2. Wash buffer B: $20 \mathrm{Mm}$ Tris, $1.0 \mathrm{M} \mathrm{NaCl}, 0.1 \%$ Tween-20, Ph 8.0

3. Elution buffer $\mathrm{A}: 0.1 \mathrm{M}$ glycine, $\mathrm{Ph} 2.9$

4. Elution buffer B: $0.5 \mathrm{M}$ glycine, Ph 2.9

5. Neutralization buffer: $1.0 \mathrm{M}$ Tris, $\mathrm{Ph} 9.5$

6. Fraction collector and tubes

\section{PAG Concentration}

1. Polyethylene glycol

2. 1,000 molecular weight dialysis tubing

3. Media buffer: $1.8 \mathrm{Mm} \mathrm{CaCl}_{2}, 0.000247 \mathrm{Mm}$ Fe$\left(\mathrm{NO}_{3}\right)_{3} \cdot 9 \mathrm{H}_{2} \mathrm{O}, 0.8 \mathrm{Mm} \mathrm{MgSO}_{4}, 5.3 \mathrm{Mm} \mathrm{KCl}, 44.5 \mathrm{Mm}$ $\mathrm{NaHCO}_{3}, 110.3 \mathrm{Mm} \mathrm{NaCl}, 0.9 \mathrm{Mm} \mathrm{NaH} \mathrm{PO}_{4}-\mathrm{H}_{2} \mathrm{O}, \mathrm{Ph} 7.6$.

\section{Methods}

\section{Homogenization of Cotyledonary Extract}

1. Homogenize frozen cotyledons in cotyledon homogenization buffer.

2. Place cotyledon extract into 50,000 molecular weight dialysis tubing and dialyze in dialysis buffer $A$ at $4^{\circ} \mathrm{C}$ overnight.

3. Dialyze cotyledon extract in Buffer $\mathrm{A}$ at $4^{\circ} \mathrm{C}$ for at least 12 hours. Repeat this dialysis using Buffers B-E in that order.

4. Transfer cotyledon extract to centrifuge bottle and centrifuge the solution at $8,000 \mathrm{~g}$ for 10 minutes to remove insoluble debris, keep supernatant and discard pellet.

5. Save aliquot to quantify amount of PAG.

\section{Ammonium Sulfate Precipitation of Cotyledonary Extract}

1. Pour supernatant into beaker containing a stir bar and place on magnetic stirrer.

2. While sample is stirring, slowly add saturated ammonium sulfate to bring concentration to $40 \%$ saturation.

3. Once total volume of ammonium sulfate is added and thoroughly mixed, move beaker to $4^{\circ} \mathrm{C}$ overnight.

4. Transfer to centrifuge bottle and centrifuge the precipitate at $8,000 \mathrm{~g}$ for 10 minutes.

5. Pour supernatant into a beaker containing a stir bar and then re-suspend pellet in re-suspension buffer and store at $4^{\circ} \mathrm{C}$ for later use.

6. Place beaker on magnetic stirrer. While sample is stirring, slowly add saturated ammonium sulfate to bring concentration to $80 \%$ saturation.

7. Once total volume of ammonium sulfate is added and thoroughly mixed, move beaker to $4^{\circ} \mathrm{C}$ overnight.

8. Transfer to centrifuge bottle and centrifuge the precipitate at $8,000 \mathrm{~g}$ for 10 minutes.

9. Pour supernatant into fresh bottle being sure not to disturb the pellet and store at $4^{\circ} \mathrm{C}$ for later use. 
10. Re-suspend the pellet in re-suspension buffer, this will be the $80 \%$ ammonium sulfate cotyledon extract.

11. Place cotyledon extract into 50,000 molecular weight dialysis tubing and dialyze in dialysis buffer B at $4^{\circ} \mathrm{C}$ for at least 12 hours. Repeat dialysis in dialysis buffer $B$ at least 5-7 times to remove salts.

12. Transfer dialysis tubing to dialysis buffer $\mathrm{C}$ and dialyze at $4^{\circ} \mathrm{C}$ for at least 12 hours. Repeat dialysis 2 3 times.

13. Save aliquot to quantify amount of PAG.

\section{Preparation of Affinity Chromatography Column}

1. Bovine PAG antibody is biotinylated in dialysis buffer $B$ with a 60 -fold molar excess of EZ-Link NHSLC-LC biotin at $4^{\circ} \mathrm{C}$ for 3 hours on a tube rotator.

2. Non-reacted biotinylation reagent is removed by repeated dialysis with dialysis buffer $B$.

3. To form the column matrix, $50 \mathrm{mg}$ of biotinylated anti-PAG monoclonal (L4) is incubated with $50 \mathrm{MI}$ Agarose Avidin $D$ at room temperature for 3 hours on a tube rotator.

4. Load avidin matrix into the column and wash with equilibration buffer to remove unbound biotinylated anti-PAG antibody.

\section{Affinity Chromatography Purification of PAGs}

1. Wash the column with 40 column volumes of wash buffer $A$.

2. Load the column with $200 \mathrm{ml}$ of dialyzed $80 \%$ Ammonium Sulfate-precipitated material from section 3.2.

3. Wash the column with 10 column volumes of wash buffer $A$.

4. Wash the column with 5-10 column volumes of wash buffer $B$.

5. Wash the column with 25-30 column volumes of wash buffer $A$.

6. Elute column with 12 column volumes of elution buffer $A$ and 7 column volumes of elution buffer $B$.

7. Neutralize the Ph of the eluted fractions with neutralization buffer immediately upon collection from the column.

8. Wash the column with $10-20$ column volumes of wash buffer $A$ and begin loading the column again if PAG is still present in cotyledonary extract.

9. Analyze the fractions by dot blot with anti-PAG sera to identify fractions containing PAG.

10. Combine PAG positive fractions into glass container.

11. Add E-64, EDTA, PMSF, Azide to purified PAG to prevent proteolysis.

12. Store at $4^{\circ} \mathrm{C}$.

13. Save aliquot to quantify amount of PAG eluted off of column.

\section{PAG Concentration}

1. Place purified PAG into a glass container.

2. Put polyethylene glycol (PEG $15,000-20,000$ molecular weight) into 1,000 molecular weight dialysis tubing and place into the glass container holding the PAG to withdraw water from the PAG solution via osmosis.

3. Concentrate at $4^{\circ} \mathrm{C}$ for approximately 30 hours, changing out the polyethylene glycol filled dialysis tubing 5 times.

4. Save aliquot to quantify final concentration of PAG.

5. Analyze the concentrated PAG by sodium dodecyl sulfate (SDS)-polyacrylamide gel electrophoresis (PAGE), to determine purity via Sypro Red (Figure 11) staining

6. Dialyze concentrated PAG in media buffer for use in endometrial tissue culture. 


\section{Notes}

1. In order to calculate the amount of ammonium sulfate, use the EnCor Biotechnology, Inc. Ammonium Sulfate Calculator. http://www.encorbio.com/protocols/AM-SO4.htm

2. When centrifuging the cotyledon mixture, it is important to keep in mind the centrifuge bottles must be balanced by mass, not volume.

3. It is often a good idea to repack the matrix between the first wash step and the loading step. With such a great amount of solution being run across the column, 'channels' can be created in the matrix. When this occurs, the flow dynamics of the column will become altered and the column will also suffer a decrease in apparent binding capacity.

4. Place enough of the neutralization buffer in each collection tube prior to the elution step to neutralize the solution as it is collected so that the purified proteins are exposed to the low $\mathrm{Ph}$ for as short a time as possible.

5. When concentrating the purified PAG, put enough polyethylene glycol into the 1,000 molecular weight dialysis tubing to ensure protein is being concentrated; however, do not put so much into the tube that it breaks during the concentration process.

6. An in-house PAG ELISA was used to quantify PAGs. The PAG ELISA seems to be the best method to determine protein concentration of purified PAG when compared to BCA, Lowry, and Bradford assays.

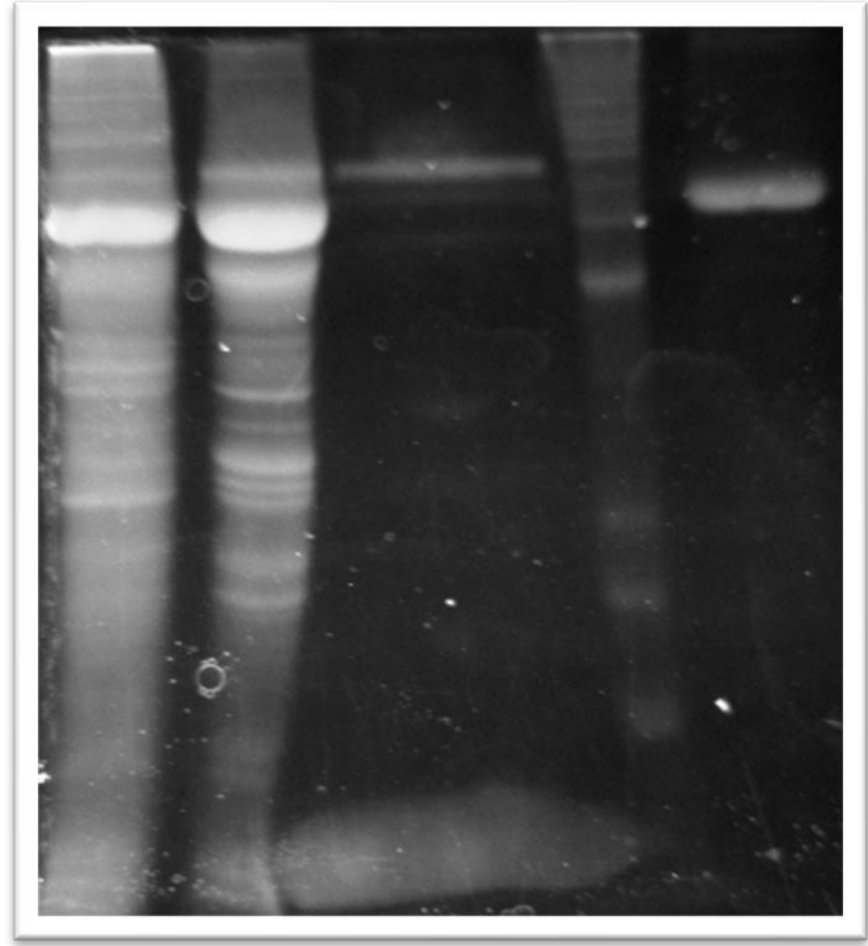

Figure 11. Analysis of the purification of PAGs from cotyledonary extract by SDS-PAGE and Sypro Red staining. Lane 1: beginning material (product of section 1); Lane 2: 80\% ammonium sulfated cotyledonary extract (product of section 2); Lane 3: eluted, concentrated material (product of section 4 and 5); Lane 4: standard to compare protein sizes; Lane 5: bovine serum albumin 


\section{APPENDIX II}

\section{Sandwich ELISA for the detection of PAGs}

\section{Summary}

Purification of the PAGs via affinity chromatography is a helpful tool in rapidly collecting a relatively pure pool of the target glycoprotein. However, depending on the size of the tissue, the number of tissues used for extraction, and the stage of pregnancy from which they were collected, the final yield can vary. Additionally, the final extract is never $100 \%$ pure PAGs. There is always additional protein that has not been removed, such as bovine serum albumin, which makes determining the PAG concentration via total protein content quite inaccurate. To better determine the yield PAG within the final placental extract an ELISA was performed to specifically measure the concentration of PAGs. The PAG ELISA was developed by Green et al. (2005). It uses anti-PAG monoclonal antibodies that recognize different placental binucleate cell-specific PAGs for a more accurate prediction of total PAG concentrations. This would then allow for a more accurate and consistent treatment of the cultured endometrial explants.

\section{Materials}

\section{Plate preparations}

1. 96-well, high binding ELISA plate

2. $0.1 \mathrm{M}$ sodium bicarbonate $\mathrm{Ph} 9.5$

\section{Assay buffers and wash buffers}

1. Blocking buffer: $1.5 \%(\mathrm{w} / \mathrm{v})$ nonfat dried milk dissolved in $0.1 \mathrm{M}$ sodium bicarbonate buffer Ph9.5

2. TBST: $20 \mathrm{Mm}$ Tris base, $150 \mathrm{Mm} \mathrm{NaCl}, 0.05 \%(\mathrm{v} / \mathrm{v})$ Tween-20, $0.001 \% \mathrm{w} / \mathrm{v}$ Thimerosal

3. Wash buffer: $150 \mathrm{Mm} \mathrm{NaCl}, 0.05 \%(v / v)$ Tween-20, $0.001 \%(\mathrm{w} / \mathrm{v})$ Thimerosal

4. AP buffer: $50 \mathrm{Mm}$ Tris base, $100 \mathrm{Mm} \mathrm{NaCl}, 1 \mathrm{mg} / \mathrm{Ml} \mathrm{MgCl}_{2}$

5. Alkaline phosphate substrate: $1 \mathrm{mg} / \mathrm{Ml}$-Nitrophenyl phosphate (PNPP; Thermo Scientific)

6. Nonpregnant heifer serum

\section{Antibodies and other reagents}

1. Sheep-anti-Mouse Fc antibody (Jackson ImmunoResearch)

2. Anti-bovine PAG monoclonal antibodies A6, J2, and L4 
3. PAG standard curve

4. Rabbit-anti-bovine PAG polyclonal antibody

5. Goat-anti-rabbit antibody conjugated to alkaline phosphatase (Jackson ImmunoResearch)

\section{Hardware needed}

1. Single or multi-channel pipettors

2. Pipette tips

3. Solution basins

4. Plate washer (optional)

5. Paper towels

6. Plate reader

\section{$\underline{\text { Methods }}$}

1. Add $100 \mathrm{ul}$ of $10 \mathrm{ug} / \mathrm{ml}$ of Sheep-anti-Mouse antibodies in $0.1 \mathrm{M}$ Sodium bicarbonate Ph9.5 to the wells of a 96-well ELISA plate. Leave at 4 degrees overnight.

2. Dump the solution and pat the plate on paper towels to remove excess solution from the wells. Add $200 \mu \mathrm{l}$ per well of blocking buffer. Put on rocking platform (if available) for $\geq 1 \mathrm{hr}$ at room temperature.

3. Add $0.5 \mathrm{ug} / \mathrm{ml}$ of each monoclonal antibody (J2, A6, and L4) to a solution of $70 \%(\mathrm{v} / \mathrm{v}) \mathrm{TBST}+$ $30 \%(\mathrm{v} / \mathrm{v}$ ) blocking solution (NFDM solution from previous step).

4. Dump the block from the plate and pat dry. Add 100 ul per well of the monoclonal antibody solution. Incubate on a rocking platform for $1 \mathrm{hr}$ at room temperature.

5. Dump the plate and pat it dry. Add $50 \mu l$ per well of TBST + blocking solutions (same as from step 3 ) to wells $A$ through $F$ to keep the wells moist. Add $100 \mu$ l of Nonpregnant heifer serum (NPHS) to rows $\mathrm{G}$ and $\mathrm{H}$. Add $100 \mu$ l of each test sample to rows A through $\mathrm{F}$ with (at least) duplicates oriented horizontally. Add $50 \mu$ l of a standard curve diluted in TBST + blocking solutions to rows $G$ and $H$. The blank for the plate will be wells $H 11$ \& $H 12$.

6. Put at 4 degrees on a rocking platform overnight. Alternatively, put the plate in an incubated shaker and shake at 750rpm and 37 degrees Celsius for 2 hours.

7. Dump the plate and pat dry. Wash the plate extensively with a plate washer or by using a spray bottle. Add $100 \mu$ l of anti-Bovine PAG polyclonal antibody in TBST + block with $2 \%$ NPHS. Incubated at room temp for $1 \mathrm{hr}$ on a rocking platform.

8. Dump and pat out the blocking solution. Wash the plate extensively with a plate washer or by using a spray bottle. Add 100 $\mu$ l of a 1:2000 dilution of Goat-anti-Rabbit antibody conjugated to alkaline phosphatase in Alkaline Phosphatase (AP) buffer. Incubated at room temp for $30 \mathrm{~min}$ on a rocking platform. 
9. Dump and pat out the blocking solution. Wash the plate extensively with a plate washer or by using a spray bottle.

10. Develop color by adding $100 \mu \mathrm{l}$ of $1 \mathrm{mg} / \mathrm{ml}$ PNPP dissolved in AP buffer. Plates are typically ready to read in 5-15 mins.

11. Read the plate in a plate reader at a $405 \mathrm{~nm}$ wavelength.

\section{$\underline{\text { Notes }}$}

1. Just about any non-specific protein solution can be used as a blocking reagent.

2. The monoclonal antibodies used here are the same that were used by Green et al. (2005)

3. The PAG standard curve stock used for this assay started at $800 \mathrm{ng} / \mathrm{Ml}$. From that a serial dilution was performed to obtain an 11-point curve with a 'blank'/negative control at the end.

4. The 'blank' wells consist solely of TBST + blocking buffers with no PAG from the standard curve or from serum samples

5. The anti-bovine PAG polyclonal antibody was made in-house by the laboratory of Jonathan Green

6. Horseradish peroxidase (HRP)-conjugated anti-rabbit antibody can be used in place of the AP-conjugated antibody. If an HRP-conjugated antibody is used, then TMB substrate should be used in place of PNPP and the colorimetric reaction should be stopped with $1 \mathrm{M} \mathrm{HCl}$ after the desired time. Plate should be read at a $450 \mathrm{~nm}$ wavelength.

\section{APPENDIX III}

SDS-polyacrylamide gel electrophoresis (PAGE) for detection and evaluation of bovine cotyledonary PAG extracts

\section{Summary}

Antibodies recognize and bind to specific epitopes on their target antigen that consist of either a particular amino acid sequence and/or a particular conformational structure. Either of these epitope types could be displayed on intact, properly folded proteins or misfolded or partially degraded proteins. That means that affinity chromatography of the PAGs does not ensure that the purified protein is intact. To verify that the purified protein was not degraded, the purified PAGs were assessed using a standard SDS-PAGE protocol. Intact PAG glycoproteins present as a single band between 62 and $65 \mathrm{kDa}$.

Degraded protein would present as a smear or as multiple faint bands of varying sizes. This method also 
serves as a semi-quantitative assay to roughly assess the amount of intact protein compared to the amount of degraded protein, if any.

\section{$\underline{\text { Materials }}$}

\section{Stacking gel}

1. Acrylamide/Bis-acrylamide (Thermo Scientific)

2. $1.5 \mathrm{M}$ Tris- $\mathrm{HCl} \mathrm{Ph} 8.8$

3. $10 \%(w / v)$ SDS (Fisher)

4. TEMED (Fisher)

5. $10 \%(w / v)$ ammonium persulfate (AP) (Fisher)

Recipe for a $5 \mathrm{Ml}$ stacker gel:

$\mathrm{H} 2 \mathrm{O}$

$2.975 \mathrm{ml}$

$0.5 \mathrm{M}$ Tris- $\mathrm{HCl}, \mathrm{Ph} 6.8$

$1.25 \mathrm{ml}$

$10 \%(w / v)$ SDS

$0.05 \mathrm{ml}$

Acrylamide/Bis-acrylamide

$0.67 \mathrm{ml}$

$(30 \% / 0.8 \% \mathrm{w} / \mathrm{v})$

$10 \%(\mathrm{w} / \mathrm{v})$ ammonium

$0.05 \mathrm{ml}$

persulfate (AP)

TEMED

$0.005 \mathrm{ml}$

\section{Loading buffer}

1. $10 \%(w / v)$ SDS

2. Dithiothreitol or beta-mercapto-ethanol

3. Glycerol

4. Tris-HCl Ph 6.8

5. Bromophenolblue

Receipt for a $5 x$ loading buffer stock:

$10 \% \mathrm{w} / \mathrm{v} \quad$ SDS

$10 \mathrm{Mm} \quad$ Dithiothreitol, or beta-mercapto-ethanol

$20 \% \mathrm{v} / \mathrm{v} \quad$ Glycerol

$0.2 \mathrm{M} \quad$ Tris- $\mathrm{HCl}, \mathrm{Ph} 6.8$

$0.05 \% \mathrm{w} / \mathrm{v} \quad$ Bromophenolblue 


\section{Resolving gel}

1. Acrylamide/Bis-acrylamide (Thermo Scientific)

2. $1.5 \mathrm{M}$ Tris Ph 8.8

3. $10 \%(w / v)$ SDS (Fisher)

4. $10 \%$ ammonium persulfate (AP) (Fisher)

5. TEMED (Fisher)

Recipe for a $10 \mathrm{Ml}$ resolving gel:

\begin{tabular}{|l|c|c|c|c|c|}
\hline $\begin{array}{l}\text { Acrylamide } \\
\text { Percentage }\end{array}$ & $6 \%$ & $8 \%$ & $10 \%$ & $12 \%$ & $15 \%$ \\
\hline $\mathrm{H} 20$ & $5.2 \mathrm{ml}$ & $4.6 \mathrm{ml}$ & $3.8 \mathrm{ml}$ & $3.2 \mathrm{ml}$ & $2.2 \mathrm{ml}$ \\
\hline $\begin{array}{l}\text { Acrylamide/Bis- } \\
\text { acrylamide } \\
(30 \% / 0.8 \% \mathrm{w} / \mathrm{v})\end{array}$ & $2 \mathrm{ml}$ & $2.6 \mathrm{ml}$ & $3.4 \mathrm{ml}$ & $4 \mathrm{ml}$ & $5 \mathrm{ml}$ \\
\hline $1.5 \mathrm{M} \mathrm{Tris}(\mathrm{Ph}=8.8)$ & $2.6 \mathrm{ml}$ & $2.6 \mathrm{ml}$ & $2.6 \mathrm{ml}$ & $2.6 \mathrm{ml}$ & $2.6 \mathrm{ml}$ \\
\hline $10 \%(\mathrm{w} / \mathrm{v}) \mathrm{SDS}$ & $0.1 \mathrm{ml}$ & $0.1 \mathrm{ml}$ & $0.1 \mathrm{ml}$ & $0.1 \mathrm{ml}$ & $0.1 \mathrm{ml}$ \\
\hline $\begin{array}{l}10 \%(\mathrm{w} / \mathrm{v}) \\
\text { ammonium } \\
\text { persulfate (AP) }\end{array}$ & $100 \mu \mathrm{l}$ & $100 \mu \mathrm{l}$ & $100 \mu \mathrm{l}$ & $100 \mu \mathrm{l}$ & $100 \mu \mathrm{l}$ \\
\hline TEMED & $10 \mu \mathrm{l}$ & $10 \mu \mathrm{l}$ & $10 \mu \mathrm{l}$ & $10 \mu \mathrm{l}$ & $10 \mu \mathrm{l}$ \\
\hline
\end{tabular}

\section{Running buffer}

1. $25 \mathrm{Mm}$ Tris- $\mathrm{HCl}$

2. 200Mm Glycine

\section{Methods}

1. Make the separating gel:

- Set the casting frames (clamp two glass plates in the casting frames) on the casting stands.

- Prepare the gel solution (as described above) in a separate small beaker.

- Swirl the solution gently but thoroughly.

- Pipet appropriate amount of separating gel solution (listed above) into the gap between the glass plates.

- To make the top of the separating gel be horizontal, fill in water (either isopropanol) into the gap until a overflow.

- Wait for 20-30min to let it gelate.

2. Make the stacking gel:

- Discard the water and you can see separating gel left.

- Pipet in stacking gel until a overflow. 
- Insert the well-forming comb without trapping air under the teeth. Wait for 20-30min to let it gelate.

- Make sure a complete gelation of the stacking gel and take out the comb. Take the glass plates out of the casting frame and set them in the cell buffer dam. Pour the running buffer (electrophoresis buffer) into the inner chamber and keep pouring after overflow until the buffer surface reaches the required level in the outer chamber.

3. Prepare the samples:

- Mix your samples with sample buffer (loading buffer).

- Heat them in boiling water for 5-10 min.

4. Load prepared samples into wells and make sure not to overflow. Don't forget loading protein marker into the first lane. Then cover the top and connect the anodes.

5. Set an appropriate volt and run the electrophoresis when everything's done.

6. As for the total running time, stop SDS-PAGE running when the downmost sign of the protein marker (if no visible sign, inquire the manufacturer) almost reaches the foot line of the glass plate (Figure 11). Generally, about 1 hour for a $120 \mathrm{~V}$ voltage and a $12 \%$ separating gel. For a separating gel possessing higher percentage of acrylamide, the time will be longer.

\section{$\underline{\text { Notes }}$}

- Higher concentration of AP and TEMED lead to faster gelation. I often double the normal amount to speed up the gelation time.

- The SDS-PAGE gels run in the validation of the PAGs for these experiments was $12.5 \%$

- Loading buffer should be diluted to a $1 x$ concentration into the desired sample

- Protein molecular weight marker that was used in these experiments was BenchMark Prestained protein ladder from Invitrogen

- This protocol is borrowed from http://www.assay-protocol.com/molecularbiology/electrophoresis/denaturing-page.html 


\section{APPENDIX IV}

Assessment of changes in gene transcription of MMPs and prostaglandin synthases by real time PCR in bovine endometrial explants exposed to PAGs

\section{Summary}

The PAGs have been predicted to plays roles in matrix turnover at the utero-placental interface and/or in the maternal stroma (Wooding et al. 2005; Wallace et al. 2019). Several of the PAGs have been shown to be active aspartic proteinases (B. P. V. L. Telugu and Green 2008; B. P. V. L. Telugu et al. 2010) and the PAGs have previously been shown to upregulated expression of several MMPs in bovine endometrial explants (R. M. Wallace et al. 2019). Previous research has also implicated the PAGs in luteotrophic mechanisms by upregulating production of $\mathrm{PGE}_{2}$ in ovine endometrial explants (Weems et al. 2003). To assess these potential functions of the PAGs, real time PCR (Qpcr) was performed analyze the changes in transcripts for several MMPs as well as some prostaglandin synthesis-related genes in endometrial explants exposed to bovine PAGs.

\section{Materials}

\section{Hardware}

1. Single or multichannel pipettor

2. Pipette tips

3. 96-well PCR reaction plate (Applied Biosystems)

4. Optical adhesive film (Applied Biosystems)

5. Quant Studio 3 real-time PCR system (Applied Biosystems)

\section{PCR Reagents}
1. Primers (Table 1)
2. Power SYBR Green PCR master mix (Applied Biosystems)
3. Template Cdna
4. Nuclease-free water

\section{Methods}

1. To one well of the reaction plate add 1.25ng of DNA template, $6.25 \mathrm{UI}$ of SYBR Green master mix, forward and reverse primers at optimized concentrations, and enough nuclease-free water for a final reaction volume of $12.5 \mathrm{UI}$.

2. Apply optical adhesive film over the reaction plate to seal each well. 
3. Plate the plate in the Qpcr reader and run under the following cycling conditions: $95^{\circ} \mathrm{C}$ for $10 \mathrm{~min} ; 40$ cycles of $95^{\circ} \mathrm{C}$ for 15 seconds and $58^{\circ} \mathrm{C}$ for 1 minute.

4. A dissociation curve was included in each Qpcr run to ensure specificity of the amplicons.

\section{$\underline{\text { Notes/Results }}$}

1. Primer sequences and concentrations used in each assay are listed in Table 1

2. In nonpregnant and pregnant samples, MMP1, MMP3, and MMP13 were upregulated after 48 and 72 hours (Figure $12 \mathrm{~A}$ and $\mathrm{B}$ )

3. In nonpregnant samples, MMP12 was upregulated on after 48 hours and MMP14 was upregulated only after 72 hours (Figure 12A)

4. In pregnant samples, MMP12 was downregulated only after 72 hours and there was no change in MMP14 abundance (Figure 12B)

5. In nonpregnant samples, PTGES, PTGS1, and PTGS2 were upregulated only after 48 hours (Figure 13)

6. In pregnant samples, PTGES and PTGS1 were downregulated after 48 hours and PTGS2 was upregulated after 48 and 72 hours (Figure 14)

$\mathrm{A}_{3.000}$
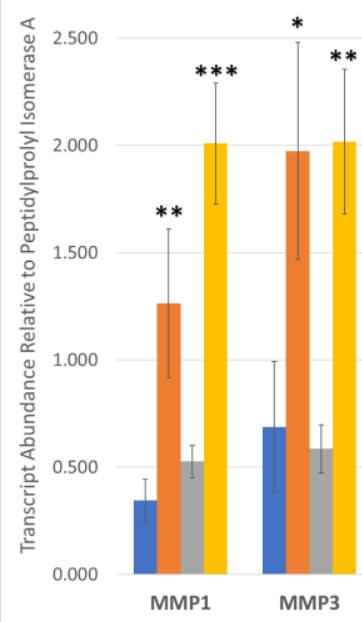
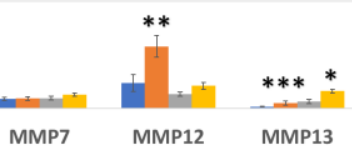

B
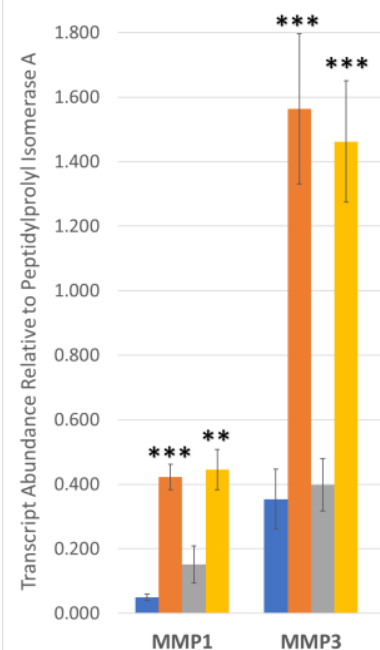

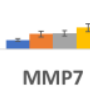

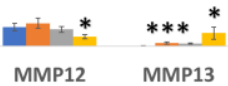

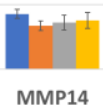

Figure 12. Relative transcript abundance of MMPs in nonpregnant and pregnant endometrium after exposure to PAGs for 48 and $72 \mathrm{hr}$

Target transcript abundance of MMP1, MMP3, MMP7, MMP12, MMP13, and MMP14 in endometrial explants from nonpregnant (A) and pregnant (B) heifers exposed to PAGs for 48 (blue and orange bars) and 72 hours (grey and yellow bars). Data was quantified by RT-PCR and the results were normalized to PPIA. Data are presented as mean \pm SEM. Blue bar: $48 \mathrm{hr}$ nontreated endometrium; Orange bar: 48hr PAG-treated endometrium; Grey bar: $72 \mathrm{hr}$ nontreated endometrium; Yellow bar: $72 \mathrm{hr}$ PAG-treated endometrium. ${ }^{*} \mathrm{P}<0.05 ;{ }^{* *} \mathrm{P}<0.01 ;{ }^{* *} \mathrm{P}<0.001$. 

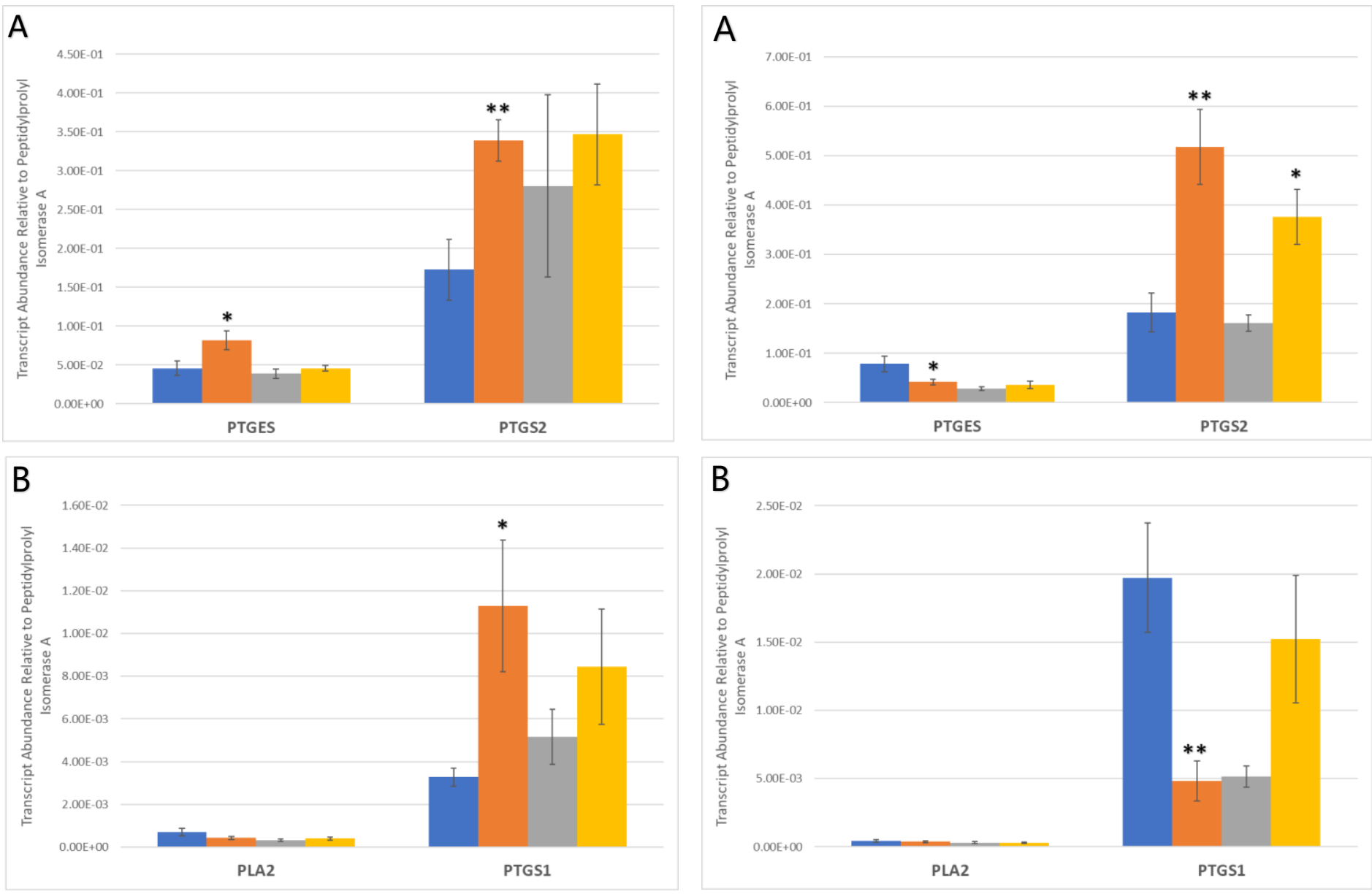

Figure 13. Relative transcript abundance of prostaglandin synthesis-related genes in nonpregnant endometrium after exposure to PAGs for 48 and $\mathbf{7 2 h r}$

Target transcript abundance of several prostaglandin synthesis-related genes in endometrial explants from nonpregnant heifers exposed to PAGs for 48 (blue and orange bars) and 72 hours (grey and yellow bars). Data was quantified by RT-PCR and the results were normalized to PPIA. Data are presented as mean \pm SEM. Blue bar: 48hr nontreated endometrium; Orange bar: 48hr PAG-treated endometrium; Grey bar: $72 \mathrm{hr}$ nontreated endometrium; Yellow bar: $72 \mathrm{hr}$ PAG-treated endometrium. ${ }^{*} \mathrm{P}<0.05 ;{ }^{* *} \mathrm{P}<0.01 ;{ }^{* * *} \mathrm{P}<0.001$.

Figure 14. Relative transcript abundance of prostaglandin synthesis-related genes in pregnant endometrium after exposure to PAGs for $\mathbf{4 8}$ and $\mathbf{7 2 h r}$

Target transcript abundance of several prostaglandin synthesis-related genes in endometrial explants from pregnant heifers exposed to PAGs for 48 (blue and orange bars) and 72 hours (grey and yellow bars). Data was quantified by RT-PCR and the results were normalized to PPIA. Data are presented as mean \pm SEM. Blue bar: 48hr nontreated endometrium; Orange bar: 48hr PAG-treated endometrium; Grey bar: $72 \mathrm{hr}$ nontreated endometrium; Yellow bar: 72hr PAG-treated endometrium. ${ }^{*} \mathrm{P}<0.05 ;{ }^{*} \mathrm{P}<0.01 ;{ }^{*} * \mathrm{P}<0.001$. 


\section{APPENDIX V}

Caspase-3 and PCNA immunofluorescent (IF) assessment of apoptosis in endometrial explants exposed to PAGs

\section{Summary}

Explant culture system have their advantages over cell culture system. The main advantage is that the responses seen are more representative of the in vivo tissue. However, there are some disadvantages when it comes to maintaining a tissue outside of the body. There is no more blood supply, so unless the culture medium is changed often, the supply of oxygen and nutrients to the tissue is limited, especially to the cells at the center of the explant. This can be avoided by making the explant tissues small, but apoptosis is bound to occur and can negatively affect the results of the assay. As a result, tissue explant systems are not usually maintained for long because cells begin to die. Because the current study utilized an explant culture system that maintained the tissues outside of the body for up to four days, it was important to assess the degree of apoptosis that may have occurred. To assess this, immunofluorescence (IF) was performed for the presence of an apoptosis marker, activated Caspase-3, and a proliferation marker, PCNA, with fixed endometrial explants either treated or not treated with PAG for 24 and 96 hours.

\section{Materials}

1. Formalin-fixed, paraffin-embedded endometrial explants

2. Antigen retrieval: $10 \mathrm{mM}$ Tris base, $1 \mathrm{mM}$ EDTA, $0.05 \%(\mathrm{v} / \mathrm{v})$ Tween-20 pH 9.0 (Tris-EDTA)

3. Serum block buffer: $2 \%(\mathrm{v} / \mathrm{v})$ horse serum, $1 \%(\mathrm{w} / \mathrm{v})$ bovine serum albumin, $0.1 \%(\mathrm{v} / \mathrm{v})$ Triton $\mathrm{X}-$ $100,0.05 \%(v / v)$ Tween-20, PBS

4. Primary antibody: Anti-activated Caspase-3 antibody (Abcam)

5. Primary antibody: Anti-PCNA antibody (Abcam)

6. Secondary antibody: Anti-mouse IgG antibody conjugated to Alexa Fluor 488 (Jackson ImmunoResearch)

7. Secondary antibody: Anti-rabbit IgG antibody conjugated to Alexa Fluor 594 (Jackson ImmunoResearch)

8. 4',6-diamidino-2-phenylindole (DAPI)

9. ProLong Diamond Antifade Mountant (Invitrogen)

10. Ethanol

11. Xylene 
12. Phosphate buffered saline (PBS)

13. Phosphate buffered saline with Tween-20 (PBST)

\section{Methods}

\section{Preparing the slides}

1. Paraffin-embedded tissues were sectioned $5 \mu \mathrm{m}$ thick and adhered to charged glass slides

2. 2-3 sections were adhered to each slide

\section{Staining the tissues}

1. Tissue sections were deparaffinized in 3 changes of xylene, 5 minutes each

2. Tissue section were than gradually rehydrated by submerging in decreasing concentrations of ethanol starting at $100 \%$ ethanol and ending in deionized water

3. Tissues were washed in PBST for 3 minutes

4. Heat-mediated antigen retrieval was performed by submerging tissue sections in a staining dish containing Tris-EDTA buffer that had pre-heated in a steamer to $95-100^{\circ} \mathrm{C}$. Tissues were incubated for 20 minutes in the hot Tris-EDTA in the steamer.

5. With the tissue sections still submerged, the Tris-EDTA staining dish was taken out of the steamer and allowed to cool at room temperature for about 20 minutes.

6. Non-specific binding was blocked by covering the slides with serum blocking buffer until each of the tissues were covered. Tissues were incubated with the blocking buffer for 30 minutes at room temperature in a moisture chamber.

7. Blocking buffer was dumped off of the slides and they were then washed briefly by submerging in PBST.

8. Slides were carefully patted dry and a hydrophobic barrier was drawn around each tissue section using a PAP pen.

9. Appropriate primary antibodies were then applied to each tissue section individually at manufacturer recommended concentrations and were allowed to incubate at $37^{\circ} \mathrm{C}$ for 1 hour in a moisture chamber.

10. Antibody was removed from slides and the slides were washed in 2 changes of PBST, 2-3 minutes each.

11. Appropriate fluorescent secondary antibodies were applied to each tissue section individually at manufacturers recommended concentrations and allowed to incubate for 30 minutes at room temperature in a moisture chamber in the dark.

12. Secondary antibodies were removed from slides and the slides were washed in 2 changes of PBST, 2-3 minutes each.

13. Nuclei were counterstained by applying DAPI (up to $10 \mu \mathrm{g} / \mathrm{mL}$ ) to each tissue individually and incubating for 5-7 minutes at room temperature in a moisture chamber in the dark.

14. Slides were then briefly washed in PBST and quickly dehydrated through $100 \%$ ethanol.

15. Coverslips were mounted over the slides with Prolong Diamond Antifade and the mountant was allowed to cure overnight in a dry place in the dark.

16. The next morning the slides were examined under a fluorescent microscope. 


\section{Notes/Results}

1. Figure 15 shows results from a single representative animal

2. There were no differences between pregnant and nonpregnant samples

3. There were no differences between control (Figure 15A-C and G-I) and PAG-treated (Figure 15D$\mathrm{F}$ and $\mathrm{J}-\mathrm{L}$ ) samples

4. Presence of activated caspase-3 and PCNA were low in the 24 hour samples of all animals and mainly in the subepithelial stroma

5. By 96 hours, activated caspase- 3 was more abundance as was PCNA

6. Activated caspase- 3 seemed to be more prevalent in the stroma, indicating an increase in apoptosis

7. In the $96 \mathrm{hr}$ samples PCNA was also more abundant and was localized in the luminal and glandular epithelia 

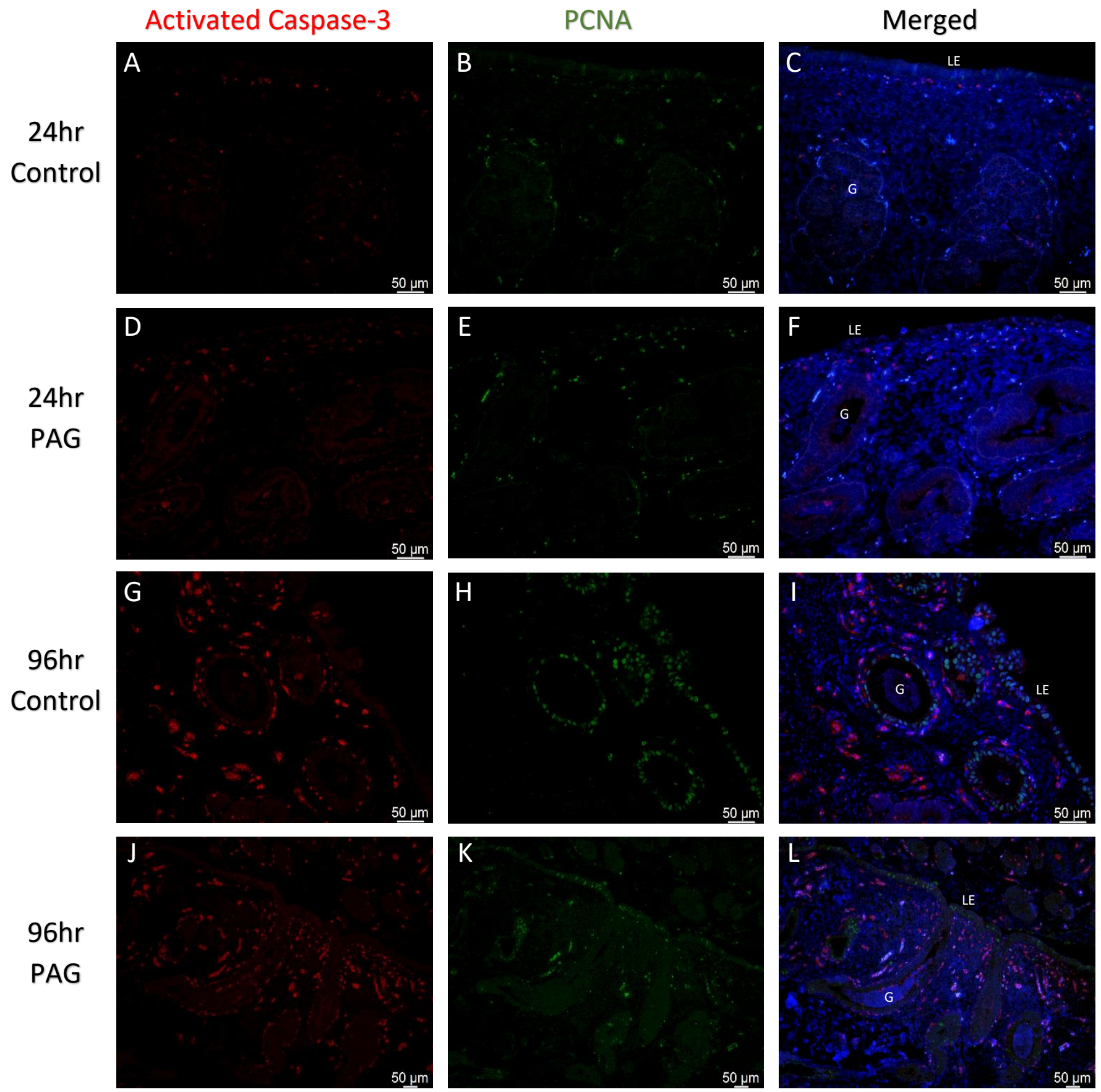

Figure 15 Immunofluorescent staining for activated caspase-3 and PCNA in endometrial explants exposed to PAGs for 24 and 96 hours

Bovine endometrial explants cultured for 24 hours (A-F) and 96 hours (G-L) either in the absence (A$\mathrm{C}$ and $\mathrm{G}-\mathrm{I}$ ) or in the presence (D-F and J-L) of bovine PAGs. Tissues were stained for activated caspase-3 (red; left column) to assess the degree of cellular apoptosis. Tissues were also stained for PCNA (green; center column) to assess the amount of proliferation. Nuclei were counterstained with DAPI (blue). Results were similar between treatment and pregnancy status so results from a single animal is shown here as a representative. $G$, gland; $L E$, luminal epithelium 


\section{BIBLIOGRAPHY}

Adams, Nicholas M., Simon Grassmann, and Joseph C. Sun. 2020. "Clonal Expansion of Innate and Adaptive Lymphocytes." Nature Reviews Immunology 20 (11): 694-707.

https://doi.org/10.1038/s41577-020-0307-4.

Addison, Christina L., Thomas O. Daniel, Marie D. Burdick, Hua Liu, Jan E. Ehlert, Ying Ying Xue, Linda Buechi, Alfred Walz, Ann Richmond, and Robert M. Strieter. 2000. "The CXC Chemokine Receptor 2, CXCR2, Is the Putative Receptor for ELR+ CXC Chemokine-Induced Angiogenic Activity." The Journal of Immunology 165 (9): 5269-77. https://doi.org/10.4049/jimmunol.165.9.5269.

Al-Alem, Linah, Muraly Puttabyatappa, Kathy Rosewell, Mats Brännström, James Akin, Jeffrey Boldt, Ken Muse, and Thomas E. Curry. 2015. "Chemokine Ligand 20: A Signal for Leukocyte Recruitment During Human Ovulation?" Endocrinology 156 (9): 3358-69. https://doi.org/10.1210/en.20141874.

Allen, W. R., D. W. Hamilton, and R. M. Moor. 1973. "The Origin of Equine Endometrial Cups. II. Invasion of the Endometrium by Trophoblast." The Anatomical Record 177 (4): 485-501. https://doi.org/10.1002/ar.1091770403.

Allman, David, Jin Li, and Richard R. Hardy. 1999. "Commitment to the B Lymphoid Lineage Occurs before DH-JH Recombination." The Journal of Experimental Medicine 189 (4): 735-40.

Alvarez-Oxiley, Andrea V., Noelita M. de Sousa, and Jean-François Beckers. 2008. "Native and Recombinant Bovine Placental Lactogens." Reproductive Biology 8 (2): 85-106. https://doi.org/10.1016/S1642-431X(12)60006-0.

Amoroso, E. C. 1952. "Placentation." In Marshall's Physiology of Reproduction, 2:127-311. Longman, Green and Co., London.

Andoh, Kiyohiko, Asami Nishimori, Ryosuke Sakumoto, Ken-Go Hayashi, and Shinichi Hatama. 2020. "The Chemokines CCL2 and CXCL10 Produced by Bovine Endometrial Epithelial Cells Induce Migration of Bovine B Lymphocytes, Contributing to Transuterine Transmission of BLV Infection." Veterinary Microbiology 242 (March): 108598. https://doi.org/10.1016/j.vetmic.2020.108598.

Aristizábal, Beatriz, and Ángel González. 2013. Innate Immune System. Autoimmunity: From Bench to Bedside [Internet]. El Rosario University Press. https://www.ncbi.nlm.nih.gov/books/NBK459455/.

Arosh, J. A., S. K. Banu, S. Kimmins, P. Chapdelaine, L. A. Maclaren, and M. A. Fortier. 2004. "Effect of Interferon-Tau on Prostaglandin Biosynthesis, Transport, and Signaling at the Time of Maternal Recognition of Pregnancy in Cattle: Evidence of Polycrine Actions of Prostaglandin E2." Endocrinology 145 (11): 5280-93. https://doi.org/10.1210/en.2004-0587.

Ashworth, Cheryl J., and Fuller W. Bazer. 1989. "Changes in Ovine Conceptus and Endometrial Function Following Asynchronus Embryo Transfer or Administration of Progesterone1." Biology of Reproduction 40 (2): 425-33. https://doi.org/10.1095/biolreprod40.2.425.

Asselin, Eric, Fuller W. Bazer, and Michel A. Fortier. 1997. "Recombinant Ovine and Bovine Interferons $\tau$ Regulate Prostaglandin Production and Oxytocin Response in Cultured Bovine Endometrial Cells1." Biology of Reproduction 56 (2): 402-8. https://doi.org/10.1095/biolreprod56.2.402.

Asselin, Eric, Greg A. Johnson, Thomas E. Spencer, and Fuller W. Bazer. 2001. "Monocyte Chemotactic Protein-1 and -2 Messenger Ribonucleic Acids in the Ovine Uterus: Regulation by Pregnancy, Progesterone, and Interferon- $\tau$." Biology of Reproduction 64 (3): 992-1000. https://doi.org/10.1095/biolreprod64.3.992.

Atkinson, Y. H., K. J. Gogolin-Ewens, E. F. Hounsell, M. J. Davies, M. R. Brandon, and R. F. Seamark. 1993. "Characterization of Placentation-Specific Binucleate Cell Glycoproteins Possessing a Novel 
Carbohydrate. Evidence for a New Family of Pregnancy-Associated Molecules." Journal of Biological Chemistry 268 (35): 26679-85. https://doi.org/10.1016/S0021-9258(19)74366-X.

Austin, Kathy J., Cathy P. King, Judith E. Vierk, R. Garth Sasser, and Thomas R. Hansen. 1999. "PregnancySpecific Protein B Induces Release of an Alpha Chemokine in Bovine Endometrium." Endocrinology 140 (1): 542-45. https://doi.org/10.1210/endo.140.1.6608.

Bachelder, Robin E., Melissa A. Wendt, and Arthur M. Mercurio. 2002. "Vascular Endothelial Growth Factor Promotes Breast Carcinoma Invasion in an Autocrine Manner by Regulating the Chemokine Receptor CXCR4." Cancer Research 62 (24): 7203-6.

Baggiolini, Marco. 1998. "Chemokines and Leukocyte Traffic." Nature 392 (6676): 565-68. https://doi.org/10.1038/33340.

Bairagi, S., K. E. Quinn, A. R. Crane, R. L. Ashley, P. P. Borowicz, J. S. Caton, R. R. Redden, A. T. GrazulBilska, and L. P. Reynolds. 2016. "Maternal Environment and Placental Vascularization in Small Ruminants." Theriogenology, Proceedings of the 18th ICAR, 86 (1): 288-305. https://doi.org/10.1016/j.theriogenology.2016.04.042.

Baker, Erica, and E. H. Morgan. 1973. "Placental Iron Transfer in the Cat." The Journal of Physiology 232 (3): 485-501.

Balestrieri, Maria Luisa, Antonio Balestrieri, Francesco Paolo Mancini, and Claudio Napoli. 2008. "Understanding the Immunoangiostatic CXC Chemokine Network." Cardiovascular Research 78 (2): 250-56. https://doi.org/10.1093/cvr/cvn029.

Barlic, Jana, Joseph D. Andrews, Alyson A. Kelvin, Steven E. Bosinger, Mark E. DeVries, Luoling Xu, Tomas Dobransky, Ross D. Feldman, Stephen S. G. Ferguson, and David J. Kelvin. 2000. "Regulation of Tyrosine Kinase Activation and Granule Release through $\beta$-Arrestin by CXCR1." Nature Immunology 1 (3): 227-33. https://doi.org/10.1038/79767.

Bartol, F. F., A. A. Wiley, T. E Spencer, N. H. Ing, T. L. Ott, and F. W Bazer. 1997. "Progestin Exposure from Birth: Epigenetic Induction of a Unique Adult Uterine Phenotype in Sheep-a Glandless Endometrium" 56 (Suppl 1): 133 (abstract 202).

Bazer, F. W., and W. W. Thatcher. 1977. "Theory of Maternal Recognition of Pregnancy in Swine Based on Estrogen Controlled Endocrine versus Exocrine Secretion of Prostaglandin F2alpha by the Uterine Endometrium." Prostaglandins 14 (2): 397-400. https://doi.org/10.1016/00906980(77)90185-x.

Bazer, Fuller W. 1975. "Uterine Protein Secretions: Relationship to Development of the Conceptus." Journal of Animal Science 41 (5): 1376-82. https://doi.org/10.2527/jas1975.4151376x.

Bazer, Fuller W., Robert C. Burghardt, Greg A. Johnson, Thomas E. Spencer, and Guoyao Wu. 2008. "Interferons and Progesterone for Establishment and Maintenance of Pregnancy: Interactions among Novel Cell Signaling Pathways." Reproductive Biology 8 (3): 179-211. https://doi.org/10.1016/S1642-431X(12)60012-6.

Bazer, Fuller W., Thomas E. Spencer, Greg A. Johnson, Robert C. Burghardt, and Guoyao Wu. 2009. "Comparative Aspects of Implantation." Reproduction 138 (2): 195-209. https://doi.org/10.1530/REP-09-0158.

Bazer, Fuller W., Guoyao Wu, Thomas E. Spencer, Greg A. Johnson, Robert C. Burghardt, and Kayla Bayless. 2010. "Novel Pathways for Implantation and Establishment and Maintenance of Pregnancy in Mammals." Molecular Human Reproduction 16 (3): 135-52. https://doi.org/10.1093/molehr/gap095.

Behrendt, Jan Hillern, Carlos Hermosilla, Martin Hardt, Klaus Failing, Horst Zahner, and Anja Taubert. 2008. “PMN-Mediated Immune Reactions against Eimeria Bovis." Veterinary Parasitology 151 (2): 97-109. https://doi.org/10.1016/j.vetpar.2007.11.013. 
Belperio, John A., Michael P. Keane, Douglas A. Arenberg, Christina L. Addison, Jan E. Ehlert, Marie D. Burdick, and Robert M. Strieter. 2000. "CXC Chemokines in Angiogenesis." Journal of Leukocyte Biology 68 (1): 1-8. https://doi.org/10.1189/jlb.68.1.1.

Bériot, Mathilde, Aline Flora Tchimbou, Olimpia Barbato, Jean-François Beckers, and Noelita M. de Sousa. 2014. "Identification of Pregnancy-Associated Glycoproteins and Alpha-Fetoprotein in Fallow Deer (Dama Dama) Placenta." Acta Veterinaria Scandinavica 56: 4. https://doi.org/10.1186/1751-0147-56-4.

Birkedal-Hansen, H., W.G.I. Moore, M.K. Bodden, L.J. Windsor, B. Birkedal-Hansen, A. DeCarlo, and J.A. Engler. 1993. "Matrix Metalloproteinases: A Review." Critical Reviews in Oral Biology \& Medicine 4 (2): 197-250. https://doi.org/10.1177/10454411930040020401.

Bonapace, Laura, Marie-May Coissieux, Jeffrey Wyckoff, Kirsten D. Mertz, Zsuzsanna Varga, Tobias Junt, and Mohamed Bentires-Alj. 2014. "Cessation of CCL2 Inhibition Accelerates Breast Cancer Metastasis by Promoting Angiogenesis." Nature 515 (7525): 130-33. https://doi.org/10.1038/nature13862.

Boos, A., V. Janssen, and C. Mulling. 2003. "Proliferation and Apoptosis in Bovine Placentomes during Pregnancy and around Induced and Spontaneous Parturition as Well as in Cows Retaining the Fetal Membranes." Reproduction 126 (4): 469-80. https://doi.org/10.1530/rep.0.1260469.

Boshier, D. P. 1969. "A Histological and Histochemical Examination of Implantation and Early Placentome Formation in Sheep." Journal of Reproduction and Fertility 19 (1): 51-61. https://doi.org/10.1530/jrf.0.0190051.

Bowen, Jeffery A., Fuller W. Bazer, and Robert C. Burghardt. 1996. "Spatial and Temporal Analyses of Integrin and Muc-1 Expression in Porcine Uterine Epithelium and Trophectoderm in Vivo1." Biology of Reproduction 55 (5): 1098-1106. https://doi.org/10.1095/biolreprod55.5.1098.

Brandt, Gretchen A., Tina E. Parks, Gary Killian, Alan D. Ealy, and Jonathan A. Green. 2007. "A Cloning and Expression Analysis of Pregnancy-Associated Glycoproteins Expressed in Trophoblasts of the White-Tail Deer Placenta." Molecular Reproduction and Development 74 (11): 1355-62. https://doi.org/10.1002/mrd.20669.

Brännström, Mats, and Anders Enskog. 2002. "Leukocyte Networks and Ovulation." Journal of Reproductive Immunology 57 (1): 47-60. https://doi.org/10.1016/S0165-0378(02)00009-8.

Brännström, Mats, J. Frederick Woessner JR, Robert D. Koos, Christopher H. J. Sear, and William J. Lemaire. 1988. "Inhibitors of Mammalian Tissue Collagenase and Metalloproteinases Suppress Ovulation in the Perfused Rat Ovary*." Endocrinology 122 (5): 1715-21. https://doi.org/10.1210/endo-122-5-1715.

Bukulmez, Orhan, and Aydin Arici. 2000. "Leukocytes in Ovarian Function." Human Reproduction Update 6 (1): 1-15. https://doi.org/10.1093/humupd/6.1.1.

Burghardt, Robert C., Greg A. Johnson, Laurie A. Jaeger, Hakhyun Ka, Jane E. Garlow, Thomas E. Spencer, and Fuller W. Bazer. 2002. "Integrins and Extracellular Matrix Proteins at the Maternal-Fetal Interface in Domestic Animals." Cells Tissues Organs 172 (3): 202-17. https://doi.org/10.1159/000066969.

Burt, Trevor D. 2013. "Fetal Regulatory T Cells and Peripheral Immune Tolerance in Utero: Implications for Development and Disease." American Journal of Reproductive Immunology (New York, N.Y. : 1989) 69 (4): 346-58. https://doi.org/10.1111/aji.12083.

Butler, J. E., W. C. Hamilton, R. G. Sasser, C. A. Ruder, G. M. Hass, and R. J. Williams. 1982. "Detection and Partial Characterization of Two Bovine Pregnancy-Specific Proteins." Biology of Reproduction 26 (5): 925-33. https://doi.org/10.1095/biolreprod26.5.925.

Buza, Joram J., Yasuyuki Mori, Abusaleh M. Bari, Hirokazu Hikono Aodon-geril, Sachiyo Hirayama, Yujing Shu, and Eiichi Momotani. 2003. "Mycobacterium Avium Subsp. Paratuberculosis Infection Causes Suppression of RANTES, Monocyte Chemoattractant Protein 1, and Tumor Necrosis 
Factor Alpha Expression in Peripheral Blood of Experimentally Infected Cattle." Infection and Immunity 71 (12): 7223-27. https://doi.org/10.1128/IAI.71.12.7223-7227.2003.

Cantor, Alan B., Samuel G. Katz, and Stuart H. Orkin. 2002. "Distinct Domains of the GATA-1 Cofactor FOG-1 Differentially Influence Erythroid versus Megakaryocytic Maturation." Molecular and Cellular Biology 22 (12): 4268-79. https://doi.org/10.1128/MCB.22.12.4268-4279.2002.

Cantor, Alan B., and Stuart H. Orkin. 2002. "Transcriptional Regulation of Erythropoiesis: An Affair Involving Multiple Partners." Oncogene 21 (21): 3368-76. https://doi.org/10.1038/sj.onc.1205326.

Cao, Jian, Alnawaz Rehemtulla, Wadie Bahou, and Stanley Zucker. 1996. "Membrane Type Matrix Metalloproteinase 1 Activates Pro-Gelatinase A without Furin Cleavage of the N-Terminal Domain*." Journal of Biological Chemistry 271 (47): 30174-80. https://doi.org/10.1074/jbc.271.47.30174.

Cappello, Paola, Cristiana Caorsi, Marita Bosticardo, Stefania De Angelis, Francesco Novelli, Guido Forni, and Mirella Giovarelli. 2004. "CCL16/LEC Powerfully Triggers Effector and Antigen-Presenting Functions of Macrophages and Enhances T Cell Cytotoxicity." Journal of Leukocyte Biology 75 (1): 135-42. https://doi.org/10.1189/jlb.0403146.

Carter, Anthony M., and Allen C. Enders. 2004. "Comparative Aspects of Trophoblast Development and Placentation." Reproductive Biology and Endocrinology 2 (1): 46. https://doi.org/10.1186/14777827-2-46.

Chaplin, David D. 2010. "Overview of the Immune Response." The Journal of Allergy and Clinical Immunology 125 (2 Suppl 2): S3-23. https://doi.org/10.1016/j.jaci.2009.12.980.

Chau, S. E., P. Murthi, M. H. Wong, G. S. Whitley, S. P. Brennecke, and R. J. Keogh. 2013. "Control of Extravillous Trophoblast Function by the Eotaxins CCL11, CCL24 and CCL26." Human Reproduction 28 (6): 1497-1507. https://doi.org/10.1093/humrep/det060.

Chavatte-Palmer, P., N. de Sousa, P. Laigre, S. Camous, A. A. Ponter, J. -F. Beckers, and Y. Heyman. 2006. "Ultrasound Fetal Measurements and Pregnancy Associated Glycoprotein Secretion in Early Pregnancy in Cattle Recipients Carrying Somatic Clones." Theriogenology 66 (4): 829-40. https://doi.org/10.1016/j.theriogenology.2006.01.061.

Christenson, L. K., D. B. Farley, L. H. Anderson, and S. P. Ford. 1994. "Luteal Maintenance during Early Pregnancy in the Pig: Role for Prostaglandin E2." Prostaglandins 47 (1): 61-75. https://doi.org/10.1016/0090-6980(94)90075-2.

Cocchi, Fiorenza, Anthony L. DeVico, Alfredo Garzino-Demo, Suresh K. Arya, Robert C. Gallo, and Paolo Lusso. 1995. "Identification of RANTES, MIP-1 $\alpha$, and MIP-1 $\beta$ as the Major HIV-Suppressive Factors Produced by CD8+ T Cells." Science 270 (5243): 1811-15. https://doi.org/10.1126/science.270.5243.1811.

Constant, F., S. Camous, P. Chavatte-Palmer, Y. Heyman, N. de Sousa, C. Richard, J. F. Beckers, and M. Guillomot. 2011. "Altered Secretion of Pregnancy-Associated Glycoproteins during Gestation in Bovine Somatic Clones." Theriogenology 76 (6): 1006-21. https://doi.org/10.1016/j.theriogenology.2011.04.029.

Dahm-Kähler, Pernilla, Manda Ghahremani, Anna-Karin Lind, Karin Sundfeldt, and Mats Brännström. 2009. "Monocyte Chemotactic Protein-1 (MCP-1), Its Receptor, and Macrophages in the Perifollicular Stroma during the Human Ovulatory Process." Fertility and Sterility 91 (1): 231-39. https://doi.org/10.1016/j.fertnstert.2007.07.1330.

Davies, D R. 1990. "The Structure and Function of the Aspartic Proteinases." Annual Review of Biophysics and Biophysical Chemistry 19 (1): 189-215. https://doi.org/10.1146/annurev.bb.19.060190.001201.

De, Rajat K., and Namrata Tomar, eds. 2014. Immunoinformatics. Vol. 1184. Methods in Molecular Biology. New York, NY: Springer New York. https://doi.org/10.1007/978-1-4939-1115-8. 
Del Vecchio, R. P., W. D. Sutherland, and R. G. Sasser. 1995. "Prostaglandin F2 Alpha, Progesterone and Oxytocin Production by Cultured Bovine Luteal Cells Treated with Prostaglandin E2 and Pregnancy-Specific Protein B." Prostaglandins 50 (3): 137-50. https://doi.org/10.1016/00906980(95)00108-5.

- - . 1996. "Bovine Luteal Cell Production in Vitro of Prostaglandin E2, Oxytocin and Progesterone in Response to Pregnancy-Specific Protein B and Prostaglandin F2 Alpha." Journal of Reproduction and Fertility 107 (1): 131-36. https://doi.org/10.1530/jrf.0.1070131.

DeSouza, Mary M., Gulnar A. Surveyor, Roger E. Price, JoAnne Julian, Rachele Kardon, Xinhui Zhou, Sandra Gendler, John Hilkens, and Daniel D. Carson. 2000. "MUC1/Episialin: A Critical Barrier in the Female Reproductive Tract." Journal of Reproductive Immunology 45 (2): 127-58. https://doi.org/10.1016/S0165-0378(99)00046-7.

Dietl, J., A. Hönig, U. Kämmerer, and L. Rieger. 2006. "Natural Killer Cells and Dendritic Cells at the Human Feto-Maternal Interface: An Effective Cooperation?" Placenta 27 (4): 341-47. https://doi.org/10.1016/j.placenta.2005.05.001.

Dilly, M., N. Hambruch, S. Shenavai, G. Schuler, R. Froehlich, J. -D. Haeger, G. R. Ozalp, and C. Pfarrer. 2011. "Expression of Matrix Metalloproteinase (MMP)-2, MMP-14 and Tissue Inhibitor of Matrix Metalloproteinase (TIMP)-2 during Bovine Placentation and at Term with or without Placental Retention." Theriogenology 75 (6): 1104-14. https://doi.org/10.1016/j.theriogenology.2010.11.019.

Ding, Lei, and Sean J. Morrison. 2013. "Haematopoietic Stem Cells and Early Lymphoid Progenitors Occupy Distinct Bone Marrow Niches." Nature 495 (7440): 231-35. https://doi.org/10.1038/nature11885.

Dosogne, H, C Burvenich, A. E Freeman, M. E Kehrli Jr, J. C Detilleux, J Sulon, J. -F Beckers, and D Hoeben. 1999. "Pregnancy-Associated Glycoprotein and Decreased Polymorphonuclear Leukocyte Function in Early Post-Partum Dairy Cows." Veterinary Immunology and Immunopathology 67 (1): 47-54. https://doi.org/10.1016/S0165-2427(98)00215-3.

Doyle, Julie, Shirley A. Ellis, Grace M. O'Gorman, Ines Maria Aparicio Donoso, Patrick Lonergan, and Trudee Fair. 2009. "Classical and Non-Classical Major Histocompatibility Complex Class I Gene Expression in in Vitro Derived Bovine Embryos." Journal of Reproductive Immunology 82 (1): 4856. https://doi.org/10.1016/j.jri.2009.06.125.

Duello, Theresa M., John C. Byatt, and Robert D. Bremel. 1986. "Immunohistochemical Localization of Placental Lactogen in Binucleate Cells of Bovine Placentomes *." Endocrinology 119 (3): 135155. https://doi.org/10.1210/endo-119-3-1351.

Duffy, Diane M., CheMyong Ko, Misung Jo, Mats Brannstrom, and Thomas E. Curry. 2019. "Ovulation: Parallels With Inflammatory Processes." Endocrine Reviews 40 (2): 369-416. https://doi.org/10.1210/er.2018-00075.

Dunlap, Kathrin A., Massimo Palmarini, David L. Adelson, and Thomas E. Spencer. 2005. "Sheep Endogenous Betaretroviruses (EnJSRVs) and the Hyaluronidase 2 (HYAL2) Receptor in the Ovine Uterus and Conceptus." Biology of Reproduction 73 (2): 271-79. https://doi.org/10.1095/biolreprod.105.039776.

Dunlap, Kathrin A., Massimo Palmarini, Mariana Varela, Robert C. Burghardt, Kanako Hayashi, Jennifer L. Farmer, and Thomas E. Spencer. 2006. "Endogenous Retroviruses Regulate Periimplantation Placental Growth and Differentiation." Proceedings of the National Academy of Sciences of the United States of America 103 (39): 14390-95. https://doi.org/10.1073/pnas.0603836103.

Dunn, Ben M. 2002. "Structure and Mechanism of the Pepsin-Like Family of Aspartic Peptidases." Chemical Reviews 102 (12): 4431-58. https://doi.org/10.1021/cr010167q.

Ealy, Alan D., and Lydia K. Wooldridge. 2017. "The Evolution of Interferon-Tau." Reproduction 154 (5): F1-10. https://doi.org/10.1530/REP-17-0292. 
Ealy, Alan D., and Qi En Yang. 2009. "REVIEW ARTICLE: Control of Interferon-Tau Expression During Early Pregnancy in Ruminants." American Journal of Reproductive Immunology 61 (2): 95-106. https://doi.org/10.1111/j.1600-0897.2008.00673.x.

Egen, T. E., A. D. Ealy, L. A. Landon, R. M. Roberts, and J. A. Green. 2009. "Autoimmunization of Ewes against Pregnancy-Associated Glycoproteins Does Not Interfere with the Establishment and Maintenance of Pregnancy." Animal 3 (6): 850-57. https://doi.org/10.1017/S1751731109004145.

Enders, A. C. 1965. "A COMPARATIVE STUDY OF THE FINE STRUCTURE OF THE TROPHOBLAST IN SEVERAL HEMOCHORIAL PLACENTAS." The American Journal of Anatomy 116 (January): 29-67. https://doi.org/10.1002/aja.1001160103.

Enders, A. C., and A. M. Carter. 2006. "Comparative Placentation: Some Interesting Modifications for Histotrophic Nutrition - A Review." Placenta, Trophoblast Research Placenta: From Molecule to Morphology to Mother, 27 (April): 11-16. https://doi.org/10.1016/j.placenta.2005.10.013.

Enders, A. C., and I. K. Liu. 1991. "Trophoblast-Uterine Interactions during Equine Chorionic Girdle Cell Maturation, Migration, and Transformation." The American Journal of Anatomy 192 (4): 366-81. https://doi.org/10.1002/aja.1001920405.

Enders, Allen C, and Thomas N Blankenship. 1999. "Comparative Placental Structure." Advanced Drug Delivery Reviews, Transplacental Drug Delivery, 38 (1): 3-15. https://doi.org/10.1016/S0169409x(99)00003-4.

Espey, L. L., and H. Lipner. 1994. "Ovulation." In Knobil E, Neil J The Physiology of Reproduction, 2nd ed., 725-80. Raven Press, New York.

Espey, Lawrence L. 1967. "Ultrastructure of the Apex of the Rabbit Graafian Follicle During the Ovulatory Process1." Endocrinology 81 (2): 267-76. https://doi.org/10.1210/endo-81-2-267.

Fazleabas, Asgerally T. 2007. "Physiology and Pathology of Implantation in the Human and Nonhuman Primate." Seminars in Reproductive Medicine 25 (6): 405-9. https://doi.org/10.1055/s-2007991037.

Filant, Justyna, Huaijun Zhou, and Thomas E. Spencer. 2012. "Progesterone Inhibits Uterine Gland Development in the Neonatal Mouse Uterus." Biology of Reproduction 86 (5). https://doi.org/10.1095/biolreprod.111.097089.

Fleming, M. W., and H. R. Gamble. 1993. "Consequences of Dose-Dependent Immunosuppression by Progesterone on Parasitic Worm Burdens in Lambs." American Journal of Veterinary Research 54 (8): 1299-1302.

Franek, A. D., L. A. Salamonsen, and A. Lopata. 1999. "Marmoset Monkey Trophoblastic Tissue Growth and Matrix Metalloproteinase Secretion in Culture." Journal of Reproduction and Fertility 117 (1): 107-14. https://doi.org/10.1530/jrf.0.1170107.

Fridlender, Zvi G., Jing Sun, Samuel Kim, Veena Kapoor, Guanjun Cheng, Leona Ling, G. Scott Worthen, and Steven M. Albelda. 2009. "Polarization of Tumor-Associated Neutrophil (TAN) Phenotype by TGF- $\beta$ : 'N1' versus 'N2' TAN." Cancer Cell 16 (3): 183-94. https://doi.org/10.1016/j.ccr.2009.06.017.

Fu, Binqing, Yonggang Zhou, Xiang Ni, Xianhong Tong, Xiuxiu Xu, Zhongjun Dong, Rui Sun, Zhigang Tian, and Haiming Wei. 2017. "Natural Killer Cells Promote Fetal Development through the Secretion of Growth-Promoting Factors." Immunity 47 (6): 1100-1113.e6. https://doi.org/10.1016/j.immuni.2017.11.018.

Furukawa, Satoshi, Yusuke Kuroda, and Akihiko Sugiyama. 2014. "A Comparison of the Histological Structure of the Placenta in Experimental Animals." Journal of Toxicologic Pathology 27 (1): 1118. https://doi.org/10.1293/tox.2013-0060. 
Gangur, Venu, Neil P. Birmingham, and Sirinart Thanesvorakul. 2002. "Chemokines in Health and Disease." Veterinary Immunology and Immunopathology 86 (3): 127-36. https://doi.org/10.1016/S0165-2427(02)00018-1.

Garbayo, Juana M., Jonathan A. Green, Mohan Manikkam, Jean-Francois Beckers, David O. Kiesling, Alan D. Ealy, and R. Michael Roberts. 2000. "Caprine Pregnancy-Associated Glycoproteins (PAG): Their Cloning, Expression, and Evolutionary Relationship to Other PAG." Molecular Reproduction and Development 57 (4): 311-22. https://doi.org/10.1002/1098-2795(200012)57:4<311::AIDMRD2>3.0.CO;2-F.

Gargett, C. E., and P. A. Rogers. 2001. "Human Endometrial Angiogenesis." Reproduction 121 (2): 18186. https://doi.org/10.1530/rep.0.1210181.

Garlow, Jane E., Hakhyun Ka, Greg A. Johnson, Robert C. Burghardt, Laurie A. Jaeger, and Fuller W. Bazer. 2002. "Analysis of Osteopontin at the Maternal-Placental Interface in Pigs." Biology of Reproduction 66 (3): 718-25. https://doi.org/10.1095/biolreprod66.3.718.

Geisert, R. D., M. T. Zavy, R. P. Wettemann, and B. G. Biggers. 1987. "Length of Pseudopregnancy and Pattern of Uterine Protein Release as Influenced by Time and Duration of Oestrogen Administration in the Pig." Reproduction 79 (1): 163-72. https://doi.org/10.1530/jrf.0.0790163.

Geisert, Rodney D., Gregory A. Johnson, and Robert C. Burghardt. 2015. "Implantation and Establishment of Pregnancy in the Pig." In Regulation of Implantation and Establishment of Pregnancy in Mammals: Tribute to 45 Year Anniversary of Roger V. Short's "Maternal Recognition of Pregnancy," edited by Rodney D. Geisert and Fuller W. Bazer, 137-63. Advances in Anatomy, Embryology and Cell Biology. Cham: Springer International Publishing. https://doi.org/10.1007/978-3-319-15856-3_8.

Geissmann, Frederic, Steffen Jung, and Dan R. Littman. 2003. "Blood Monocytes Consist of Two Principal Subsets with Distinct Migratory Properties." Immunity 19 (1): 71-82. https://doi.org/10.1016/S1074-7613(03)00174-2.

Giblin, P. A., S. T. Hwang, T. R. Katsumoto, and S. D. Rosen. 1997. "Ligation of L-Selectin on T Lymphocytes Activates Beta1 Integrins and Promotes Adhesion to Fibronectin." The Journal of Immunology 159 (7): 3498-3507.

Gifford, C. A., K. Racicot, D. S. Clark, K. J. Austin, T. R. Hansen, M. C. Lucy, C. J. Davies, and T. L. Ott. 2007a. "Regulation of Interferon-Stimulated Genes in Peripheral Blood Leukocytes in Pregnant and Bred, Nonpregnant Dairy Cows." Journal of Dairy Science 90 (1): 274-80. https://doi.org/10.3168/jds.S0022-0302(07)72628-0.

- - . 2007b. "Regulation of Interferon-Stimulated Genes in Peripheral Blood Leukocytes in Pregnant and Bred, Nonpregnant Dairy Cows." Journal of Dairy Science 90 (1): 274-80. https://doi.org/10.3168/jds.S0022-0302(07)72628-0.

Gilbert, Robert O., Sang T. Shin, Charles L. Guard, Hollis N. Erb, and Marcel Frajblat. 2005. "Prevalence of Endometritis and Its Effects on Reproductive Performance of Dairy Cows." Theriogenology 64 (9): 1879-88. https://doi.org/10.1016/j.theriogenology.2005.04.022.

Glaucia Teixeira, M., Kathy J. Austin, David J. Perry, Vern D. Dooley, Greg A. Johnson, Brian R. Francis, and Thomas R. Hansen. 1997. "Bovine Granulocyte Chemotactic Protein-2 Is Secreted by the

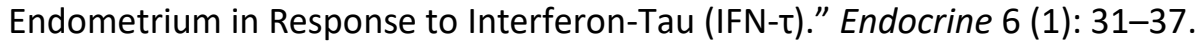
https://doi.org/10.1007/BF02738799.

Godkin, J. D., F. W. Bazer, W. W. Thatcher, and R. M. Roberts. 1984. "Proteins Released by Cultured Day 15-16 Conceptuses Prolong Luteal Maintenance When Introduced into the Uterine Lumen of Cyclic Ewes." Journal of Reproduction and Fertility 71 (1): 57-64. https://doi.org/10.1530/jrf.0.0710057.

Goodnow, C. C., J. Crosbie, S. Adelstein, T. B. Lavoie, S. J. Smith-Gill, R. A. Brink, H. Pritchard-Briscoe, J. S. Wotherspoon, R. H. Loblay, and K. Raphael. 1988. "Altered Immunoglobulin Expression and 
Functional Silencing of Self-Reactive B Lymphocytes in Transgenic Mice." Nature 334 (6184): 676-82. https://doi.org/10.1038/334676a0.

Gottshall, S L, and P J Hansen. 1992. "Regulation of Leucocyte Subpopulations in the Sheep Endometrium by Progesterone." Immunology 76 (4): 636-41.

Gouon-Evans, Valérie, and Jeffrey W. Pollard. 2001. "Eotaxin Is Required for Eosinophil Homing into the Stroma of the Pubertal and Cycling Uterus." Endocrinology 142 (10): 4515-21. https://doi.org/10.1210/endo.142.10.8459.

Gouwy, Mieke, Sofie Struyf, Julie Catusse, Paul Proost, and Jo Van Damme. 2004. "Synergy between Proinflammatory Ligands of G Protein-Coupled Receptors in Neutrophil Activation and Migration." Journal of Leukocyte Biology 76 (1): 185-94. https://doi.org/10.1189/jlb.1003479.

Gouwy, Mieke, Sofie Struyf, Frank Mahieu, Willy Put, Paul Proost, and Jo Van Damme. 2002. "The Unique Property of the CC Chemokine Regakine-1 to Synergize with Other Plasma-Derived Inflammatory Mediators in Neutrophil Chemotaxis Does Not Reside in Its NH2-Terminal Structure." Molecular Pharmacology 62 (1): 173-80.

Gray, C. Allison, David L. Adelson, Fuller W. Bazer, Robert C. Burghardt, Els N. T. Meeusen, and Thomas E. Spencer. 2004. "Discovery and Characterization of an Epithelial-Specific Galectin in the Endometrium That Forms Crystals in the Trophectoderm." Proceedings of the National Academy of Sciences of the United States of America 101 (21): 7982-87. https://doi.org/10.1073/pnas.0402669101.

Gray, C. Allison, Frank F. Bartol, Becky J. Tarleton, Anne A. Wiley, Greg A. Johnson, Fuller W. Bazer, and Thomas E. Spencer. 2001. "Developmental Biology of Uterine Glands." Biology of Reproduction 65 (5): 1311-23. https://doi.org/10.1095/biolreprod65.5.1311.

Gray, C. Allison, Frank F. Bartol, Kristin M. Taylor, Anne A. Wiley, W. Shawn Ramsey, Troy L. Ott, Fuller W. Bazer, and Thomas E. Spencer. 2000. "Ovine Uterine Gland Knock-Out Model: Effects of Gland Ablation on the Estrous Cycle1." Biology of Reproduction 62 (2): 448-56. https://doi.org/10.1095/biolreprod62.2.448.

Gray, C. Allison, Fuller W. Bazer, and Thomas E. Spencer. 2001. "Effects of Neonatal Progestin Exposure on Female Reproductive Tract Structure and Function in the Adult Ewe." Biology of Reproduction 64 (3): 797-804. https://doi.org/10.1095/biolreprod64.3.797.

Gray, C. Allison, Kristin M. Taylor, W. Shawn Ramsey, Jonathan R. Hill, Fuller W. Bazer, Frank F. Bartol, and Thomas E. Spencer. 2001. "Endometrial Glands Are Required for Preimplantation Conceptus Elongation and Survival." Biology of Reproduction 64 (6): 1608-13. https://doi.org/10.1095/biolreprod64.6.1608.

Gray, C., R. Burghardt, G. Johnson, F. Bazer, and T. Spencer. 2002. "Evidence That Absence of Endometrial Gland Secretions in Uterine Gland Knockout Ewes Compromises Conceptus Survival and Elongation." Reproduction. https://doi.org/10.1530/REP.0.1240289.

Green, Jonathan A, and Madison E Hennessy. 2018. "Pregnancy Associated Glycoproteins." In Encyclopedia of Reproduction, M. K. Skinner, 2:508-13.

Green, Jonathan A., Tina E. Parks, Mary Pavlo Avalle, Bhanu Prakash Telugu, April L. McLain, A. James Peterson, William McMillan, et al. 2005a. "The Establishment of an ELISA for the Detection of Pregnancy-Associated Glycoproteins (PAGs) in the Serum of Pregnant Cows and Heifers." Theriogenology 63 (5): 1481-1503. https://doi.org/10.1016/j.theriogenology.2004.07.011.

- - . 2005b. "The Establishment of an ELISA for the Detection of Pregnancy-Associated Glycoproteins (PAGs) in the Serum of Pregnant Cows and Heifers." Theriogenology 63 (5): 1481-1503. https://doi.org/10.1016/j.theriogenology.2004.07.011.

Green, Jonathan A., Sancai Xie, Xiao Quan, Bagna Bao, Xinsheng Gan, Nagappan Mathialagan, JeanFrançois Beckers, and R. Michael Roberts. 2000. "Pregnancy-Associated Bovine and Ovine 
Glycoproteins Exhibit Spatially and Temporally Distinct Expression Patterns During Pregnancy." Biology of Reproduction 62 (6): 1624-31. https://doi.org/10.1095/biolreprod62.6.1624.

Greenstein, J. S., R. W. Murray, and R. C. Foley. 1958. "Observations on the Morphogenesis and Histochemistry of the Bovine Pre-Attachment Placenta between 16 and 33 Days of Gestation." The Anatomical Record 132 (3): 321-41. https://doi.org/10.1002/ar.1091320308.

Griffith, Jason W., Caroline L. Sokol, and Andrew D. Luster. 2014. "Chemokines and Chemokine Receptors: Positioning Cells for Host Defense and Immunity." Annual Review of Immunology 32 (1): 659-702. https://doi.org/10.1146/annurev-immunol-032713-120145.

Gross, T. S., W. F. Williams, and E. Russek-Cohen. 1991. "Cellular Changes in the Peripartum Bovine Fetal Placenta Related to Placental Separation." Placenta 12 (1): 27-35. https://doi.org/10.1016/0143-4004(91)90507-C.

Grosser, Otto. 1909. Vergleichende Anatomie Und Entwicklungsgeschichte Der Eihäute Und Der Placenta. Braumuller, Wien und Leipzig.

-- - 1927. Frühentwicklung, Eihautbildung Und Placentation Des Menschen Und Der Säugetiere. München, J.F. Bergmann.

Gu, Long, Barbara Rutledge, Joseph Fiorillo, Catherine Ernst, lqbal Grewal, Richard Flavell, Ron Gladue, and Barrett Rollins. 1997. "In Vivo Properties of Monocyte Chemoattractant Protein-1." Journal of Leukocyte Biology 62 (5): 577-80. https://doi.org/10.1002/jlb.62.5.577.

Guillomot, M. 1995. "Cellular Interactions during Implantation in Domestic Ruminants." Journal of Reproduction and Fertility. Supplement 49: 39-51.

-- - 1999. "Changes in Extracellular Matrix Components and Cytokeratins in the Endometrium during Goat Implantation." Placenta 20 (4): 339-45. https://doi.org/10.1053/plac.1998.0385.

Guillomot, M., K. J. Betteridge, D. Harvey, and A. K. Goff. 1986. "Endocytotic Activity in the Endometrium during Conceptus Attachment in the Cow." Journal of Reproduction and Fertility 78 (1): 27-36. https://doi.org/10.1530/jrf.0.0780027.

Guillomot, M., J.-E. Fléchon, and S. Wintenberger-Torres. 1981. "Conceptus Attachment in the Ewe: An Ultrastructural Study." Placenta 2 (2): 169-81. https://doi.org/10.1016/S0143-4004(81)80021-5.

Guillomot, M., J.-E. Flechon, and S. Wintenberger-Torres. 1982. "Cytochemical Studies of Uterine and Trophoblastic Surface Coats during Blastocyst Attachment in the Ewe." Reproduction 65 (1): 1-8. https://doi.org/10.1530/jrf.0.0650001.

Guillomot, Michel, and Patrick Guay. 1982. "Ultrastructural Features of the Cell Surfaces of Uterine and Trophoblastic Epithelia during Embryo Attachment in the Cow." The Anatomical Record 204 (4): 315-22. https://doi.org/10.1002/ar.1092040404.

Guimerà, Marta, Manuel Morales-Ruiz, Wladimiro Jiménez, and Juan Balasch. 2009. “LH/HCG Stimulation of VEGF and Adrenomedullin Production by Follicular Fluid Macrophages and Luteinized Granulosa Cells." Reproductive BioMedicine Online 18 (6): 743-49. https://doi.org/10.1016/S1472-6483(10)60021-1.

Guruharsha, K. G., Mark W. Kankel, and Spyros Artavanis-Tsakonas. 2012. "The Notch Signalling System: Recent Insights into the Complexity of a Conserved Pathway." Nature Reviews. Genetics 13 (9): 654-66. https://doi.org/10.1038/nrg3272.

Guruprasad, K., T. L. Blundell, S. Xie, J. Green, B. Szafranska, R. J. Nagel, K. McDowell, C. B. Baker, and R. M. Roberts. 1996. "Comparative Modelling and Analysis of Amino Acid Substitutions Suggests That the Family of Pregnancy-Associated Glycoproteins Includes Both Active and Inactive Aspartic Proteinases." Protein Engineering 9 (10): 849-56. https://doi.org/10.1093/protein/9.10.849.

Hamilton, D. W., W. R. Allen, and R. M. Moor. 1973. "The Origin of Equine Endometrial Cups. III. Light and Electron Microscopic Study of Fully Developed Equine Endometrial Cups." The Anatomical Record 177 (4): 503-17. https://doi.org/10.1002/ar.1091770404. 
Hampton, Anne L., Anna R. Butt, Simon C. Riley, and Lois A. Salamonsen. 1995. "Tissue Inhibitors of Metalloproteinases in Endometrium of Ovariectomized Steroid-Treated Ewes and during the Estrous Cycle and Early Pregnancy." Biology of Reproduction 53 (2): 302-11. https://doi.org/10.1095/biolreprod53.2.302.

Hanna, Jacob, Debra Goldman-Wohl, Yaron Hamani, Inbal Avraham, Caryn Greenfield, Shira NatansonYaron, Diana Prus, et al. 2006. "Decidual NK Cells Regulate Key Developmental Processes at the Human Fetal-Maternal Interface." Nature Medicine 12 (9): 1065-74. https://doi.org/10.1038/nm1452.

Hansen, P. J. 1998. "Regulation of Uterine Immune Function by Progesterone-Lessons from the Sheep." Journal of Reproductive Immunology 40 (1): 63-79. https://doi.org/10.1016/S01650378(98)00035-7.

Hansen, P. J. 2013. "PHYSIOLOGY AND ENDOCRINOLOGY SYMPOSIUM: Maternal Immunological Adjustments to Pregnancy and Parturition in Ruminants and Possible Implications for Postpartum Uterine Health: Is There a Prepartum-Postpartum Nexus?" Journal of Animal Science 91 (4): 1639-49. https://doi.org/10.2527/jas.2012-5934.

Hansen, Peter J., Fuller W. Bazer, and Edward C. Segerson. 1986. "Skin Graft Survival in the Uterine Lumen of Ewes Treated With Progesterone." American Journal of Reproductive Immunology and Microbiology 12 (2): 48-54. https://doi.org/10.1111/j.1600-0897.1986.tb00062.x.

Hansen, T. R., K. J. Austin, D. J. Perry, J. K. Pru, M. G. Teixeira, and G. A. Johnson. 1999. "Mechanism of Action of Interferon-Tau in the Uterus during Early Pregnancy." Journal of Reproduction and Fertility. Supplement 54: 329-39.

Hardy, R R, C E Carmack, S A Shinton, J D Kemp, and K Hayakawa. 1991. "Resolution and Characterization of Pro-B and Pre-pro-B Cell Stages in Normal Mouse Bone Marrow." The Journal of Experimental Medicine 173 (5): 1213-25.

Hardy, Richard R., and Kyoko Hayakawa. 2001. "B Cell Development Pathways." Annual Review of Immunology 19 (1): 595-621. https://doi.org/10.1146/annurev.immunol.19.1.595.

Hartley, Suzanne B., Michael P. Cooke, David A. Fulcher, Alan W. Harris, Suzanne Cory, Antony Basten, and Christopher C. Goodnow. 1993. "Elimination of Self-Reactive B Lymphocytes Proceeds in Two Stages: Arrested Development and Cell Death." Cell 72 (3): 325-35. https://doi.org/10.1016/0092-8674(93)90111-3.

Hartley, Suzanne B., Jeffrey Crosbie, Robert Brink, Aaron B. Kantor, Antony Basten, and Christopher C. Goodnow. 1991. "Elimination from Peripheral Lymphoid Tissues of Self-Reactive B Lymphocytes Recognizing Membrane-Bound Antigens." Nature 353 (6346): 765-69. https://doi.org/10.1038/353765a0.

Harvey, M. B., K. J. Leco, M. Y. Arcellana-Panlilio, X. Zhang, D. R. Edwards, and G. A. Schultz. 1995. "Proteinase Expression in Early Mouse Embryos Is Regulated by Leukaemia Inhibitory Factor and Epidermal Growth Factor." Development (Cambridge, England) 121 (4): 1005-14.

Hashizume, Kazuyoshi. 2007. "Analysis of Uteroplacental-Specific Molecules and Their Functions During Implantation and Placentation in the Bovine." Journal of Reproduction and Development 53 (1): 1-11. https://doi.org/10.1262/jrd.18123.

Haworth, David J., Keith M. Rollyson, Patrick Silva, Eric W. Mclntush, and Gordon D. Niswender. 1998. "Messenger Ribonucleic Acid Encoding Monocyte Chemoattractant Protein-1 Is Expressed by the Ovine Corpus Luteum in Response to Prostaglandin F2a1." Biology of Reproduction 58 (1): 16974. https://doi.org/10.1095/biolreprod58.1.169.

Heap, R. B., R. J. Holdsworth, J. E. Gadsby, J. A. Laing, and D. E. Walters. 1976. "Pregnancy Diagnosis in the Cow from Milk Progesterone Concentration." The British Veterinary Journal 132 (5): 445-64. https://doi.org/10.1016/s0007-1935(17)34582-7. 
Heissig, Beate, Chiemi Nishida, Yoshihiko Tashiro, Yayoi Sato, Makoto Ishihara, Makiko Ohki, Ismael Gritli, Jeanette Rosenkvist, and Koichi Hattori. 2010. "Role of Neutrophil-Derived Matrix Metalloproteinase-9 in Tissue Regeneration." Histology and Histopathology 25 (6): 765-70. https://doi.org/10.14670/HH-25.765.

Hellberg, Pär, Peter Thomsen, Per Olof Janson, and Mats Brännström. 1991. "Leukocyte Supplementation Increases the Luteinizing Hormone-Induced Ovulation Rate in the in VitroPerfused Rat Ovary1." Biology of Reproduction 44 (5): 791-97. https://doi.org/10.1095/biolreprod44.5.791.

Helmer, S. D., P. J. Hansen, R. V. Anthony, W. W. Thatcher, F. W. Bazer, and R. M. Roberts. 1987. "Identification of Bovine Trophoblast Protein-1, a Secretory Protein Immunologically Related to Ovine Trophoblast Protein-1." Journal of Reproduction and Fertility 79 (1): 83-91. https://doi.org/10.1530/jrf.0.0790083.

Hillinger, Sven, Seok-Chul Yang, Li Zhu, Min Huang, Russell Duckett, Kimberly Atianzar, Raj K. Batra, Robert M. Strieter, Steven M. Dubinett, and Sherven Sharma. 2003. “EBV-Induced Molecule 1 Ligand Chemokine (ELC/CCL19) Promotes IFN- - -Dependent Antitumor Responses in a Lung Cancer Model." The Journal of Immunology 171 (12): 6457-65. https://doi.org/10.4049/jimmunol.171.12.6457.

Hirata, Michiko, Takashi Sato, Michiko Tsumagari, Arata Shimada, Haruo Nakano, Kazuyoshi Hashizume, and Akira Ito. 2003. "Differential Regulation of the Expression of Matrix Metalloproteinases and Tissue Inhibitors of Metalloproteinases by Cytokines and Growth Factors in Bovine Endometrial Stromal Cells and Trophoblast Cell Line BT-1 In Vitro." Biology of Reproduction 68 (4): 1276-81. https://doi.org/10.1095/biolreprod.102.006452.

Hirayama, Akiko, Reiko Adachi, Saki Otani, Tadashi Kasahara, and Kazuhiro Suzuki. 2007. "Cofilin Plays a Critical Role in IL-8-Dependent Chemotaxis of Neutrophilic HL-60 Cells through Changes in Phosphorylation." Journal of Leukocyte Biology 81 (3): 720-28. https://doi.org/10.1189/jlb.0506314.

Hoeben, Dagmar, Christian Burvenich, Anne-Marie Massart-Leën, Marc Lenjou, Griet Nijs, Dirk Van Bockstaele, and Jean-François Beckers. 1999. "In Vitro Effect of Ketone Bodies, Glucocorticosteroids and Bovine Pregnancy-Associated Glycoprotein on Cultures of Bone Marrow Progenitor Cells of Cows and Calves." Veterinary Immunology and Immunopathology 68 (2): 229-40. https://doi.org/10.1016/S0165-2427(99)00031-8.

Hoeben, Dagmar, Erica Monfardini, Geert Opsomer, Christian Burvenich, Hilde Dosogne, Aart De Kruif, and Jean-François Beckers. 2000. "Chemiluminescence of Bovine Polymorphonuclear Leucocytes during the Periparturient Period and Relation with Metabolic Markers and Bovine PregnancyAssociated Glycoprotein." Journal of Dairy Research 67 (2): 249-59. https://doi.org/10.1017/S0022029900004052.

Hori, Shohei, Takashi Nomura, and Shimon Sakaguchi. 2003. "Control of Regulatory T Cell Development by the Transcription Factor Foxp3." Science 299 (5609): 1057-61. https://doi.org/10.1126/science.1079490.

Hughes, Austin L., Jonathan A. Green, Juana M. Garbayo, and R. Michael Roberts. 2000. "Adaptive Diversification within a Large Family of Recently Duplicated, Placentally Expressed Genes." Proceedings of the National Academy of Sciences of the United States of America 97 (7): 331923.

Hughes, Austin L., Jonathan A. Green, Helen Piontkivska, and R. Michael Roberts. 2003. "Aspartic Proteinase Phylogeny and the Origin of Pregnancy-Associated Glycoproteins." Molecular Biology and Evolution 20 (11): 1940-45. https://doi.org/10.1093/molbev/msg217.

Hulboy, D. 1997. "Matrix Metalloproteinases as Mediators of Reproductive Function." Molecular Human Reproduction 3 (1): 27-45. https://doi.org/10.1093/molehr/3.1.27. 
Huston, D. P. 1997. "The Biology of the Immune System." JAMA 278 (22): 1804-14.

Hwang, S. T., M. S. Singer, P. A. Giblin, T. A. Yednock, K. B. Bacon, S. I. Simon, and S. D. Rosen. 1996. "GlyCAM-1, a Physiologic Ligand for L-Selectin, Activates Beta 2 Integrins on Naive Peripheral Lymphocytes." The Journal of Experimental Medicine 184 (4): 1343-48. https://doi.org/10.1084/jem.184.4.1343.

Hynes, Richard O. 1996. "Targeted Mutations in Cell Adhesion Genes: What Have We Learned from Them?" Developmental Biology 180 (2): 402-12. https://doi.org/10.1006/dbio.1996.0314.

Imakawa, Kazuhiko, Russell V. Anthony, Mohammad Kazemi, Keith R. Marotti, H. Gregory Polites, and R. Michael Roberts. 1987. "Interferon-like Sequence of Ovine Trophoblast Protein Secreted by Embryonic Trophectoderm." Nature 330 (6146): 377-79. https://doi.org/10.1038/330377a0.

Imakawa, Kazuhiko, Misa Imai, Akiharu Sakai, Masako Suzuki, Kentaro Nagaoka, Senkiti Sakai, Sang-Rae Lee, Kyu-Tae Chang, Sherrill E. Echternkamp, and Ronald K. Christenson. 2006. "Regulation of Conceptus Adhesion by Endometrial CXC Chemokines during the Implantation Period in Sheep." Molecular Reproduction and Development 73 (7): 850-58. https://doi.org/10.1002/mrd.20496.

Jeziorska, M., H. Nagase, L. A. Salamonsen, and D. E. Woolley. 1996. "Immunolocalization of the Matrix Metalloproteinases Gelatinase B and Stromelysin 1 in Human Endometrium throughout the Menstrual Cycle." Journal of Reproduction and Fertility 107 (1): 43-51. https://doi.org/10.1530/jrf.0.1070043.

Johansson, S., G. Svineng, K. Wennerberg, A. Armulik, and L. Lohikangas. 1997. "Fibronectin-Integrin Interactions." Frontiers in Bioscience: A Journal and Virtual Library 2 (March): d126-146. https://doi.org/10.2741/a178.

Johnson, Greg A., Fuller W. Bazer, Laurie A. Jaeger, Hakhyun Ka, Jane E. Garlow, Christiane Pfarrer, Thomas E. Spencer, and Robert C. Burghardt. 2001. "Muc-1, Integrin, and Osteopontin Expression During the Implantation Cascade in Sheep1." Biology of Reproduction 65 (3): 820-28. https://doi.org/10.1095/biolreprod65.3.820.

Johnson, Greg A, Robert C Burghardt, and Fuller W Bazer. 2014. "Osteopontin: A Leading Candidate Adhesion Molecule for Implantation in Pigs and Sheep." Journal of Animal Science and Biotechnology 5 (December). https://doi.org/10.1186/2049-1891-5-56.

Johnson, Greg A., Robert C. Burghardt, Fuller W. Bazer, and Thomas E. Spencer. 2003. "Osteopontin: Roles in Implantation and Placentation." Biology of Reproduction 69 (5): 1458-71. https://doi.org/10.1095/biolreprod.103.020651.

Johnson, Greg A., Robert C. Burghardt, Margaret M. Joyce, Thomas E. Spencer, Fuller W. Bazer, C. Allison Gray, and Christiane Pfarrer. 2003. "Osteopontin Is Synthesized by Uterine Glands and a 45-KDa Cleavage Fragment Is Localized at the Uterine-Placental Interface Throughout Ovine Pregnancy1." Biology of Reproduction 69 (1): 92-98. https://doi.org/10.1095/biolreprod.102.013573.

Kamat, Manasi M., Sreelakshmi Vasudevan, Samar A. Maalouf, David H. Townson, Joy L. Pate, and Troy L. Ott. 2016. "Changes in Myeloid Lineage Cells in the Uterus and Peripheral Blood of Dairy Heifers During Early Pregnancy." Biology of Reproduction 95 (3): 68. https://doi.org/10.1095/biolreprod.116.141069.

Kaneko, K., and S. Kawakami. 2009. "The Roles of PGF2 $\alpha$ and PGE2 in Regression of the Corpus Luteum after Intrauterine Infusion of Arcanobacterium Pyogenes in Cows." Theriogenology 71 (5): 85863. https://doi.org/10.1016/j.theriogenology.2008.10.007.

Kawamoto, Hiroshi, and Nagahiro Minato. 2004. "Myeloid Cells." The International Journal of Biochemistry \& Cell Biology 36 (8): 1374-79. https://doi.org/10.1016/j.biocel.2004.01.020.

Kehrli, Marcus, Brian Nonnecke, and James Roth. 1989. "Alterations in Bovine Neutrophil Function during the Periparturient Period." American Journal of Veterinary Research 50 (2): 207-14. 
Kelleher, Andrew M, Francesco J DeMayo, and Thomas E Spencer. 2019. "Uterine Glands: Developmental Biology and Functional Roles in Pregnancy." Endocrine Reviews 40 (5): 1424-45. https://doi.org/10.1210/er.2018-00281.

Kelsen, Steven G., Mark O. Aksoy, Yi Yang, Syed Shahabuddin, Judith Litvin, Fayez Safadi, and Thomas J. Rogers. 2004. "The Chemokine Receptor CXCR3 and Its Splice Variant Are Expressed in Human Airway Epithelial Cells." American Journal of Physiology-Lung Cellular and Molecular Physiology 287 (3): L584-91. https://doi.org/10.1152/ajplung.00453.2003.

Kijowski, Jacek, Monika Baj-Krzyworzeka, Marcin Majka, Ryan Reca, Leah A. Marquez, Melpo Christofidou-Solomidou, Anna Janowska-Wieczorek, and Mariusz Z. Ratajczak. 2001. "The SDF-1CXCR4 Axis Stimulates VEGF Secretion and Activates Integrins but Does Not Affect Proliferation and Survival in Lymphohematopoietic Cells." STEM CELLS 19 (5): 453-66. https://doi.org/10.1634/stemcells.19-5-453.

Kim, III-Hwa, and Hyun-Gu Kang. 2003. "Risk Factors for Postpartum Endometritis and the Effect of Endometritis on Reproductive Performance in Dairy Cows in Korea." Journal of Reproduction and Development 49 (6): 485-91. https://doi.org/10.1262/jrd.49.485.

Kim, Min-Su, Kwan-Sik Min, and Kazuhiko Imakawa. 2013. "Regulation of Interferon-Stimulated Gene (ISG)12, ISG15, and MX1 and MX2 by Conceptus Interferons (IFNTs) in Bovine Uterine Epithelial Cells." Asian-Australasian Journal of Animal Sciences 26 (6): 795-803. https://doi.org/10.5713/ajas.2012.12529.

Kimber, Susan J., and Catherine Spanswick. 2000. "Blastocyst Implantation: The Adhesion Cascade." Seminars in Cell \& Developmental Biology 11 (2): 77-92. https://doi.org/10.1006/scdb.2000.0154.

Kimmins, Sarah, Hai Choo Lim, and Leslie A MacLaren. 2004. "Immunohistochemical Localization of Integrin Alpha V Beta 3 and Osteopontin Suggests That They Do Not Interact during Embryo Implantation in Ruminants." Reproductive Biology and Endocrinology : RB\&E 2 (April): 19. https://doi.org/10.1186/1477-7827-2-19.

Kimmins, Sarah, and Leslie A. MacLaren. 1999. "Cyclic Modulation of Integrin Expression in Bovine Endometrium." Biology of Reproduction 61 (5): 1267-74. https://doi.org/10.1095/biolreprod61.5.1267.

Kimura, J., M. Sasaki, H. Endo, and K. Fukuta. 2004. "Anatomical and Histological Characterization of the Female Reproductive Organs of Mouse Deer (Tragulidae)." Placenta 25 (8): 705-11. https://doi.org/10.1016/j.placenta.2004.02.009.

King, B. F. 1993. "Development and Structure of the Placenta and Fetal Membranes of Nonhuman Primates." The Journal of Experimental Zoology 266 (6): 528-40. https://doi.org/10.1002/jez.1402660605.

King, G. J. 1993. "Comparative Placentation in Ungulates." The Journal of Experimental Zoology 266 (6): 588-602. https://doi.org/10.1002/jez.1402660609.

King, Lester S. 1952. "Dr. Koch's Postulates." Journal of the History of Medicine and Allied Sciences VII (4): 350-61. https://doi.org/10.1093/jhmas/VII.4.350.

Klisch, K., A. Boos, M. Friedrich, K. Herzog, M. Feldmann, N. M. Sousa, J. F. Beckers, R. Leiser, and G. Schuler. 2006. "The Glycosylation of Pregnancy-Associated Glycoproteins and Prolactin-Related Protein-I in Bovine Binucleate Trophoblast Giant Cells Changes before Parturition." Reproduction 132 (5): 791-98. https://doi.org/10.1530/REP-06-0040.

Klisch, K., E. Jeanrond, P.-C. Pang, A. Pich, G. Schuler, V. Dantzer, M. P Kowalewski, and A. Dell. 2007. "A Tetraantennary Glycan with Bisecting N-Acetylglucosamine and the Sda Antigen Is the Predominant N-Glycan on Bovine Pregnancy-Associated Glycoproteins." Glycobiology 18 (1): 4252. https://doi.org/10.1093/glycob/cwm113. 
Klisch, K., Christiane Pfarrer, Gerhard Schuler, Bernd Hoffmann, and Rudolf Leiser. 1999. "Tripolar Acytokinetic Mitosis and Formation of Feto-Maternal Syncytia in the Bovine Placentome: Different Modes of the Generation of Multinuclear Cells." Anatomy and Embryology 200 (2): 229-37. https://doi.org/10.1007/s004290050275.

Kobayashi, Yoshiro. 2008. "The Role of Chemokines in Neutrophil Biology." Frontiers in Bioscience 13 (13): 2400. https://doi.org/10.2741/2853.

Koshi, K., K. Ushizawa, K. Kizaki, T. Takahashi, and K. Hashizume. 2011. "Expression of Endogenous Retrovirus-like Transcripts in Bovine Trophoblastic Cells." Placenta 32 (7): 493-99. https://doi.org/10.1016/j.placenta.2011.04.002.

Koshi, Katsuo, Yasunori Suzuki, Yuki Nakaya, Kei Imai, Misa Hosoe, Toru Takahashi, Keiichiro Kizaki, Takayuki Miyazawa, and Kazuyoshi Hashizume. 2012. "Bovine Trophoblastic Cell Differentiation and Binucleation Involves Enhanced Endogenous Retrovirus Element Expression." Reproductive Biology and Endocrinology : RB\&E 10 (May): 41. https://doi.org/10.1186/1477-7827-10-41.

Kryczek, Ilona, Nelly Frydman, Françoise Gaudin, Roman Krzysiek, Renato Fanchin, Dominique Emilie, Salem Chouaib, Weiping Zou, and Véronique Machelon. 2005. "The Chemokine SDF-1/CXCL12 Contributes to T Lymphocyte Recruitment in Human Pre-Ovulatory Follicles and Coordinates with Lymphocytes to Increase Granulosa Cell Survival and Embryo Quality." American Journal of Reproductive Immunology 54 (5): 270-83. https://doi.org/10.1111/j.1600-0897.2005.00307.x.

Kumar, Brahma V., Thomas Connors, and Donna L. Farber. 2018. "Human T Cell Development, Localization, and Function throughout Life." Immunity 48 (2): 202-13. https://doi.org/10.1016/j.immuni.2018.01.007.

Kurtulus, Sema, Pulak Tripathi, and David A. Hildeman. 2013. "Protecting and Rescuing the Effectors: Roles of Differentiation and Survival in the Control of Memory T Cell Development." Frontiers in Immunology 3 (January): 404. https://doi.org/10.3389/fimmu.2012.00404.

Laing, J. A., and R. B. Heap. 1971. "The Concentration of Progesterone in the Milk of Cows during the Reproductive Cycle." The British Veterinary Journal 127 (8): xix-xxii. https://doi.org/10.1016/s0007-1935(17)37432-8.

Lasagni, Laura, Michela Francalanci, Francesco Annunziato, Elena Lazzeri, Stefano Giannini, Lorenzo Cosmi, Costanza Sagrinati, et al. 2003. "An Alternatively Spliced Variant of CXCR3 Mediates the Inhibition of Endothelial Cell Growth Induced by IP-10, Mig, and I-TAC, and Acts as Functional Receptor for Platelet Factor 4." Journal of Experimental Medicine 197 (11): 1537-49. https://doi.org/10.1084/jem.20021897.

Lawn, A M, A D Chiquoine, and E C Amoroso. 1969. "The Development of the Placenta in the Sheep and Goat: An Electron Microscope Study." Journal of Anatomy 105 (Pt 3): 557-78.

Le, Yingying, Ye Zhou, Pablo Iribarren, and Ji Ming Wang. 2004. "Chemokines and Chemokine Receptors: Their Manifold Roles in Homeostasis and Disease." Molecular Immunology 1 (2): 10.

LeBlanc, S. J., T. F. Duffield, K. E. Leslie, K. G. Bateman, G. P. Keefe, J. S. Walton, and W. H. Johnson. 2002. "Defining and Diagnosing Postpartum Clinical Endometritis and Its Impact on Reproductive Performance in Dairy Cows." Journal of Dairy Science 85 (9): 2223-36. https://doi.org/10.3168/jds.S0022-0302(02)74302-6.

Lee, C S, K Gogolin-Ewens, and M R Brandon. 1986. "Comparative Studies on the Distribution of Binucleate Cells in the Placentae of the Deer and Cow Using the Monoclonal Antibody, SBU-3." Journal of Anatomy 147 (August): 163-79.

Lee, C. S., F. B. Wooding, and M. R. Brandon. 1986a. "Light and Electron Microscope Immunocytochemical Studies on the Role of Binucleate Cells in Villus Growth in Goat Placentomes." Journal of Submicroscopic Cytology 18 (4): 661-72. 
- - . 1986b. "Immunogold Co-Localization of Ovine Placental Lactogen and the Antigen Recognized by the SBU-3 Monoclonal Antibody in Sheep Placental Granules." Journal of Reproduction and Fertility 78 (2): 653-62. https://doi.org/10.1530/jrf.0.0780653.

Lee, C. S., F. B. P. Wooding, and M. R. Brandon. 1986c. "Ultrastructural Immunogold Investigation of the Function and Diversity of Binucleate Cells in the Ovine Placenta Using a Monoclonal Antibody." Placenta 7 (6): 495-504. https://doi.org/10.1016/S0143-4004(86)80135-7.

Leiser, R., and B. Koob. 1993. "Development and Characteristics of Placentation in a Carnivore, the Domestic Cat." The Journal of Experimental Zoology 266 (6): 642-56. https://doi.org/10.1002/jez.1402660612.

Leung, S. T., K. Derecka, G. E. Mann, A. P. Flint, and D. C. Wathes. 2000. “Uterine Lymphocyte Distribution and Interleukin Expression during Early Pregnancy in Cows." Reproduction 119 (1): 25-33. https://doi.org/10.1530/reprod/119.1.25.

Li, Yue-Sheng, Robert Wasserman, Kyoko Hayakawa, and Richard R. Hardy. 1996. "Identification of the Earliest B Lineage Stage in Mouse Bone Marrow." Immunity 5 (6): 527-35. https://doi.org/10.1016/S1074-7613(00)80268-X.

Lijnen, H.R., J. Silence, B. Van Hoef, and D. Collen. 1998. "Stromelysin-1 (MMP-3)-Independent Gelatinase Expression and Activation in Mice." Blood 91 (6): 2045-53. https://doi.org/10.1182/blood.V91.6.2045.

Lincke, Annekathrin, Marc Drillich, and Wolfgang Heuwieser. 2007. "[Subclinical endometritis in dairy cattle and its effect on fertility--a review of recent publications]." Berliner Und Munchener Tierarztliche Wochenschrift 120 (5-6): 245-50.

Liu, Fei, and J. Lindsay Whitton. 2005. "Cutting Edge: Re-Evaluating the In Vivo Cytokine Responses of CD8+ T Cells during Primary and Secondary Viral Infections." The Journal of Immunology 174 (10): 5936-40. https://doi.org/10.4049/jimmunol.174.10.5936.

Liu, Wen-Jun, and Peter J. Hansen. 1993. "Effect of the Progesterone-Induced Serpin-Like Proteins of the Sheep Endometrium on Natural-Killer Cell Activity in Sheep and Mice." Biology of Reproduction 49 (5): 1008-14. https://doi.org/10.1095/biolreprod49.5.1008.

Liu, Yan, Yunhai Zhang, Qiuling Jiang, Man Rao, Zheya Sheng, Yu Zhang, Weihua Du, et al. 2015. "Identification of Valid Housekeeping Genes for Real-Time Quantitative PCR Analysis of Collapsed Lung Tissues of Neonatal Somatic Cell Nuclear Transfer-Derived Cattle." Cellular Reprogramming 17 (5): 360-67. https://doi.org/10.1089/cell.2015.0024.

Lobago, F., M. Bekana, H. Gustafsson, J. F. Beckers, G. Yohannes, Y. Aster, and H. Kindahl. 2009. "Serum Profiles of Pregnancy-Associated Glycoprotein, Oestrone Sulphate and Progesterone During Gestation and Some Factors Influencing the Profiles in Ethiopian Borana and Crossbred Cattle." Reproduction in Domestic Animals 44 (4): 685-92. https://doi.org/10.1111/j.14390531.2007.01049.x.

Lonergan, Pat, and Niamh Forde. 2015. "The Role of Progesterone in Maternal Recognition of Pregnancy in Domestic Ruminants." In Regulation of Implantation and Establishment of Pregnancy in Mammals: Tribute to 45 Year Anniversary of Roger V. Short's "Maternal Recognition of Pregnancy," edited by Rodney D. Geisert and Fuller W. Bazer, 87-104. Advances in Anatomy, Embryology and Cell Biology. Cham: Springer International Publishing. https://doi.org/10.1007/978-3-319-15856-3_6.

Lonergan, Pat, Niamh Forde, and Thomas Spencer. 2016. "Role of Progesterone in Embryo Development in Cattle." Reproduction, Fertility and Development 28 (2): 66-74. https://doi.org/10.1071/RD15326.

López-Gatius, F., J. M. Garbayo, P. Santolaria, J Yániz, A. Ayad, N. M. de Sousa, and J. F. Beckers. 2007. "Milk Production Correlates Negatively with Plasma Levels of Pregnancy-Associated Glycoprotein (PAG) during the Early Fetal Period in High Producing Dairy Cows with Live 
Fetuses." Domestic Animal Endocrinology 32 (1): 29-42.

https://doi.org/10.1016/j.domaniend.2005.12.007.

Luis, Tiago C., Michiko Ichii, Martijn H. Brugman, Paul Kincade, and Frank J.T. Staal. 2012. "Wnt Signaling Strength Regulates Normal Hematopoiesis and Its Deregulation Is Involved in Leukemia Development." Leukemia 26 (3): 414-21. https://doi.org/10.1038/leu.2011.387.

Luis, Tiago C., Brigitta A. E. Naber, Paul P. C. Roozen, Martijn H. Brugman, Edwin F. E. de Haas, Mehrnaz Ghazvini, Willem E. Fibbe, Jacques J. M. van Dongen, Riccardo Fodde, and Frank J. T. Staal. 2011. "Canonical Wnt Signaling Regulates Hematopoiesis in a Dosage-Dependent Fashion." Cell Stem Cell 9 (4): 345-56. https://doi.org/10.1016/j.stem.2011.07.017.

Luster, Andrew D., and Marc E. Rothenberg. 1997. "Role of the Monocyte Chemoattractant Protein and Eotaxin Subfamily of Chemokines in Allergic Inflammation." Journal of Leukocyte Biology 62 (5): 620-33. https://doi.org/10.1002/jlb.62.5.620.

Ma, Qing, Dan Jones, Paul R. Borghesani, Rosalind A. Segal, Takashi Nagasawa, Tadamitsu Kishimoto, Roderick T. Bronson, and Timothy A. Springer. 1998. "Impaired B-Lymphopoiesis, Myelopoiesis, and Derailed Cerebellar Neuron Migration in CXCR4- and SDF-1-Deficient Mice." Proceedings of the National Academy of Sciences of the United States of America 95 (16): 9448-53.

Mackay, Charles R. 2001. "Chemokines: Immunology's High Impact Factors." Nature Immunology 2 (2): 95-101. https://doi.org/10.1038/84298.

Mansouri-Attia, Nadéra, Lilian J. Oliveira, Niamh Forde, Alan G. Fahey, John A. Browne, James F. Roche, Olivier Sandra, Pierrette Reinaud, Patrick Lonergan, and Trudee Fair. 2012. "Pivotal Role for Monocytes/Macrophages and Dendritic Cells in Maternal Immune Response to the Developing Embryo in Cattle." Biology of Reproduction 87 (5). https://doi.org/10.1095/biolreprod.112.101121.

Martal, J., M.-C. Lacroix, C. Loudes, M. Saunier, and S. Wintenberger-Torrès. 1979. "Trophoblastin, an Antiluteolytic Protein Present in Early Pregnancy in Sheep." Reproduction 56 (1): 63-73. https://doi.org/10.1530/jrf.0.0560063.

Mathialagan, Nagappan, and Thomas R. Hansen. 1996. "Pepsin-Inhibitory Activity of the Uterine Serpins." Proceedings of the National Academy of Sciences of the United States of America 93 (24): 13653-58.

Medawar, P. 1952. "Some Immunological and Endocrinological Problems Raised by the Evolution of Viviparity in Vertebrates" 7: 320-38.

Medawar, P. B. 1948. "Immunity to Homologous Grafted Skin. III. The Fate of Skin Homographs Transplanted to the Brain, to Subcutaneous Tissue, and to the Anterior Chamber of the Eye." British Journal of Experimental Pathology 29 (1): 58-69.

Medzhitov, Ruslan, and Charles Jr Janeway. 2009. "Innate Immunity." Review-article. Http://Dx.Doi.Org/10.1056/NEJM200008033430506. Massachusetts Medical Society. World. August 20, 2009. https://doi.org/10.1056/NEJM200008033430506.

Menino, A. R., A. Hogan, G. A. Schultz, S. Novak, W. Dixon, and G. H. Foxcroft. 1997. "Expression of Proteinases and Proteinase Inhibitors during Embryo-Uterine Contact in the Pig." Developmental Genetics 21 (1): 68-74. https://doi.org/10.1002/(SICI)1520-6408(1997)21:1<68::AIDDVG8>3.0.CO;2-6.

Mercadante, P. M., K. M. Waters, V. R. G. Mercadante, G. C. Lamb, M. A. Elzo, S. E. Johnson, D. O. Rae, J. V. Yelich, and A. D. Ealy. 2013. "Subspecies Differences in Early Fetal Development and Plasma Pregnancy-Associated Glycoprotein Concentrations in Cattle1." Journal of Animal Science 91 (8): 3693-3701. https://doi.org/10.2527/jas.2012-6130.

Metcalf, D. 1998. "The Molecular Control of Hematopoiesis: Progress and Problems with Gene Manipulation." STEM CELLS 16 (5): 314-21. https://doi.org/10.1002/stem.160314. 
Meyer, Ashley E, Caroline A Pfeiffer, Kelsey E Brooks, Lee D Spate, Joshua A Benne, Raissa Cecil, Melissa S Samuel, et al. 2019. "New Perspective on Conceptus Estrogens in Maternal Recognition and Pregnancy Establishment in the Pig." Biology of Reproduction 101 (1): 148-61. https://doi.org/10.1093/biolre/ioz058.

Mialon, M. M., G. Renand, S. Camous, J. Martal, and F. Ménissier. 1994. "Detection of pregnancy by radioimmunoassay of a pregnancy serum protein (PSP60) in cattle." Reproduction Nutrition Development 34 (1): 65-72. https://doi.org/10.1051/rnd:19940107.

Mishra, Anil, Simon P. Hogan, James J. Lee, Paul S. Foster, and Marc E. Rothenberg. 1999. "Fundamental Signals That Regulate Eosinophil Homing to the Gastrointestinal Tract." Journal of Clinical Investigation 103 (12): 1719-27.

Miyasaka, Masayuki, and Peter McCullagh. 1982. "The Response of the Pregnant Ewe to Challenge with Foetal and Paternal Lymphocytes." Journal of Reproductive Immunology 4 (4): 207-15. https://doi.org/10.1016/0165-0378(82)90027-4.

Molteni, Raffaella, Carolina Lage Crespo, Sara Feigelson, Christian Moser, Monica Fabbri, Valentin Grabovsky, Fritz Krombach, Carlo Laudanna, Ronen Alon, and Ruggero Pardi. 2009. " $\beta$-Arrestin 2 Is Required for the Induction and Strengthening of Integrin-Mediated Leukocyte Adhesion during CXCR2-Driven Extravasation." Blood 114 (5): 1073-82. https://doi.org/10.1182/blood2008-10-183699.

Monterroso, Victor H, and Peter J Hansen. 1993. "Regulation of Bovine and Ovine Lymphocyte Proliferation by Progesterone: Modulation by Steroid Receptor Antagonists and Physiological Status." Acta Endocrinologica 129 (6): 532-35. https://doi.org/10.1530/acta.0.1290532.

Mor, Gil, Ingrid Cardenas, Vikki Abrahams, and Seth Guller. 2011. "Inflammation and Pregnancy: The Role of the Immune System at the Implantation Site." Annals of the New York Academy of Sciences 1221 (1): 80-87. https://doi.org/10.1111/j.1749-6632.2010.05938.x.

Morgan, G., F. B. P. Wooding, J. F. Beckers, and H. G. Friesen. 1989. "An Immunological CryoUltrastructural Study of a Sequential Appearance of Proteins in Placental Binucleate Cells in Early Pregnancy in the Cow." Reproduction 86 (2): 745-52. https://doi.org/10.1530/jrf.0.0860745.

Moser, Bernhard, and Pius Loetscher. 2001. "Lymphocyte Traffic Control by Chemokines." Nature Immunology 2 (2): 123-28. https://doi.org/10.1038/84219.

Mossman, H. W. 1987. Vertebrate Fetal Membranes. Rutgers University Press. https://agris.fao.org/agris-search/search.do?recordID=US201300622478.

Moussad, E E-D A, M A E Rageh, A K Wilson, R D Geisert, and D R Brigstock. 2002. "Temporal and Spatial Expression of Connective Tissue Growth Factor (CCN2; CTGF) and Transforming Growth Factor $\beta$ Type 1 (TGF-B1) at the Utero-Placental Interface during Early Pregnancy in the Pig." Molecular Pathology 55 (3): 186-92.

Mullen, Michael P., Giuliano Elia, Mark Hilliard, Mervyn H. Parr, Michael G. Diskin, Alex C. O. Evans, and Mark A. Crowe. 2012. "Proteomic Characterization of Histotroph during the Preimplantation Phase of the Estrous Cycle in Cattle." Journal of Proteome Research 11 (5): 3004-18. https://doi.org/10.1021/pr300144q.

Munger, John S., John G. Harpel, Filippo G. Giancotti, and Daniel B. Rifkin. 1998. "Interactions between Growth Factors and Integrins: Latent Forms of Transforming Growth Factor- $\beta$ Are Ligands for the Integrin Avß1." Molecular Biology of the Cell 9 (9): 2627-38.

Munoz-Suano, Alba, Alexander B. Hamilton, and Alexander G. Betz. 2011. "Gimme Shelter: The Immune System during Pregnancy." Immunological Reviews 241 (1): 20-38. https://doi.org/10.1111/j.1600-065X.2011.01002.x. 
Murthy, G. S., C. Schellenberg, and H. G. Friesen. 1982. "Purification and Characterization of Bovine Placental Lactogen*." Endocrinology 111 (6): 2117-24. https://doi.org/10.1210/endo-111-62117.

Nagaoka, Kentaro, Hisashi Nojima, Fumiko Watanabe, Kyu-Tae Chang, Ronald K. Christenson, Senkiti Sakai, and Kazuhiko Imakawa. 2003. "Regulation of Blastocyst Migration, Apposition, and Initial Adhesion by a Chemokine, Interferon $\gamma$-Inducible Protein 10 KDa (IP-10), during Early Gestation." Journal of Biological Chemistry 278 (31): 29048-56. https://doi.org/10.1074/jbc.M300470200.

Nagaoka, Kentaro, Akiharu Sakai, Hisashi Nojima, Yoshihito Suda, Yuichi Yokomizo, Kazuhiko Imakawa, Senkiti Sakai, and Ronald K. Christenson. 2003. "A Chemokine, Interferon (IFN)- $\gamma$-Inducible Protein $10 \mathrm{KDa}$, Is Stimulated by IFN- $\tau$ and Recruits Immune Cells in the Ovine Endometrium." Biology of Reproduction 68 (4): 1413-21. https://doi.org/10.1095/biolreprod.102.008912.

Nagase, Hideaki, and J. Frederick Woessner. 1999. "Matrix Metalloproteinases." Journal of Biological Chemistry 274 (31): 21491-94. https://doi.org/10.1074/jbc.274.31.21491.

Nakano, Haruo, Toru Takahashi, Kei Imai, and Kazuyoshi Hashizume. 2001. "Expression of Placental Lactogen and Cytokeratin in Bovine Placental Binucleate Cells in Culture." Cell and Tissue Research 303 (2): 263-70. https://doi.org/10.1007/s004410000316.

Ogawa, M., E. ten Boekel, and F. Melchers. 2000. "Identification of CD19(-)B220(+)c-Kit(+)Flt3/Flk2(+)Cells as Early B Lymphoid Precursors before Pre-B-I Cells in Juvenile Mouse Bone Marrow." International Immunology 12 (3): 313-24. https://doi.org/10.1093/intimm/12.3.313.

Oliveira, Lilian J., and P. J. Hansen. 2008. "Deviations in Populations of Peripheral Blood Mononuclear Cells and Endometrial Macrophages in the Cow during Pregnancy." Reproduction 136 (4): 48190. https://doi.org/10.1530/REP-08-0218.

Oliveira, Lilian J., Nadéra Mansourri-Attia, Alan G. Fahey, John Browne, Niamh Forde, James F. Roche, Patrick Lonergan, and Trudee Fair. 2013. "Characterization of the Th Profile of the Bovine Endometrium during the Oestrous Cycle and Early Pregnancy." PLOS ONE 8 (10). https://doi.org/10.1371/journal.pone.0075571.

Oliveira, Lilian J., Steve McClellan, and Peter J. Hansen. 2010. "Differentiation of the Endometrial Macrophage during Pregnancy in the Cow." PLOS ONE 5 (10). https://doi.org/10.1371/journal.pone.0013213.

Osmond, Dennis G, Antonius Rolink, and Fritz Melchers. 1998. "Murine B Lymphopoiesis: Towards a Unified Model." Immunology Today 19 (2): 65-68. https://doi.org/10.1016/S01675699(97)01203-6.

Ott, Troy L., and Craig A. Gifford. 2010. "Effects of Early Conceptus Signals on Circulating Immune Cells: Lessons from Domestic Ruminants." American Journal of Reproductive Immunology 64 (4): 24554. https://doi.org/10.1111/j.1600-0897.2010.00912.x.

Padua, Maria B., and Peter J. Hansen. 2010. "Evolution and Function of the Uterine Serpins (SERPINA14)." American Journal of Reproductive Immunology 64 (4): 265-74. https://doi.org/10.1111/j.1600-0897.2010.00901.x.

Palframan, Roger T., Paul D. Collins, Timothy J. Williams, and Sara M. Rankin. 1998. "Eotaxin Induces a Rapid Release of Eosinophils and Their Progenitors From the Bone Marrow." Blood 91 (7): 224048. https://doi.org/10.1182/blood.V91.7.2240.

Pall, Marita, Masato Mikuni, Kenrokuro Mitsube, and Mats Brännström. 2000. "Time-Dependent Ovulation Inhibition of a Selective Progesterone-Receptor Antagonist (Org 31710) and Effects on Ovulatory Mediators in the In Vitro Perfused Rat Ovary1." Biology of Reproduction 63 (6): 164247. https://doi.org/10.1095/biolreprod63.6.1642.

Papúchová, Henrieta, Torsten B. Meissner, Qin Li, Jack L. Strominger, and Tamara Tilburgs. 2019. "The Dual Role of HLA-C in Tolerance and Immunity at the Maternal-Fetal Interface." Frontiers in Immunology 10 (December): 2730. https://doi.org/10.3389/fimmu.2019.02730. 
Parkin, Jacqueline, and Bryony Cohen. 2001. "An Overview of the Immune System." The Lancet 357 (9270): 1777-89. https://doi.org/10.1016/S0140-6736(00)04904-7.

Patel, O. V., I. Domeki, N. Sasaki, T. Takahashi, M. Hirako, R. G. Sasser, and P. Humblot. 1995. "Effect of Fetal Mass, Number and Stage of Gestation on Pregnancy-Specific Protein B Concentrations in the Bovine." Theriogenology 44 (6): 827-33. https://doi.org/10.1016/0093-691X(95)00268-D.

Patel, Osman V., Osamu Yamada, Keiichiro Kizaki, Toru Takahashi, Kei Imai, and Kazuyoshi Hashizume. 2004. "Quantitative Analysis throughout Pregnancy of Placentomal and Interplacentomal Expression of Pregnancy-Associated Glycoproteins-1 and -9 in the Cow." Molecular Reproduction and Development 67 (3): 257-63. https://doi.org/10.1002/mrd.20017.

Paul, William E. 2003. Fundamental Immunology. 5th ed. Philadelphia, PA: Lippincott Williams and Wilkins.

Peltier, Morgan R., Wen-Jun Liu, and Peter J. Hansen. 2000. "Regulation of Lymphocyte Proliferation by Uterine Serpin: Interleukin-2 MRNA Production, CD25 Expression and Responsiveness to Interleukin-2 (44465)." Proceedings of the Society for Experimental Biology and Medicine 223 (1): 75-81. https://doi.org/10.1177/153537020022300110.

Pennington, J. A., L. H. Schultz, and W. F. Hoffman. 1985. "Comparison of Pregnancy Diagnosis by Milk Progesterone on Day 21 and Day 24 Postbreeding: Field Study in Dairy Cattle1." Journal of Dairy Science 68 (10): 2740-45. https://doi.org/10.3168/jds.S0022-0302(85)81160-7.

Pennington, J. A., S. L. Spahr, and J. R. Lodge. 1976. "Factors Affecting Progesterone in Milk for Pregnancy Diagnosis in Dairy Cattle." The British Veterinary Journal 132 (5): 487-96. https://doi.org/10.1016/s0007-1935(17)34586-4.

Pijnenborg, R., W. B. Robertson, I. Brosens, and G. Dixon. 1981. "Review Article: Trophoblast Invasion and the Establishment of Haemochorial Placentation in Man and Laboratory Animals." Placenta 2 (1): 71-91. https://doi.org/10.1016/S0143-4004(81)80042-2.

Pillay, Janesh, Bart P. Ramakers, Vera M. Kamp, Adele Lo Tam Loi, Siu W. Lam, Falco Hietbrink, Luke P. Leenen, Anton T. Tool, Peter Pickkers, and Leo Koenderman. 2010. "Functional Heterogeneity and Differential Priming of Circulating Neutrophils in Human Experimental Endotoxemia." Journal of Leukocyte Biology 88 (1): 211-20. https://doi.org/10.1189/jlb.1209793.

Plotkin, Stanley A. 2005. "Vaccines: Past, Present and Future." Nature Medicine 11 (Suppl 4): S5-11. https://doi.org/10.1038/nm1209.

Pohler, K. G., T. W. Geary, C. L. Johnson, J. A. Atkins, E. M. Jinks, D. C. Busch, J. A. Green, M. D. MacNeil, and M. F. Smith. 2013. "Circulating Bovine Pregnancy Associated Glycoproteins Are Associated with Late Embryonic/Fetal Survival but Not Ovulatory Follicle Size in Suckled Beef Cows." Journal of Animal Science 91 (9): 4158-67. https://doi.org/10.2527/jas.2013-6348.

Pohler, K. G., M. H. C. Pereira, F. R. Lopes, J. C. Lawrence, D. H. Keisler, M. F. Smith, J. L. M. Vasconcelos, and J. A. Green. 2016. "Circulating Concentrations of Bovine Pregnancy-Associated Glycoproteins and Late Embryonic Mortality in Lactating Dairy Herds." Journal of Dairy Science 99 (2): 1584-94. https://doi.org/10.3168/jds.2015-10192.

Puech, Carinne, Laurence Dedieu, Isabelle Chantal, and Valérie Rodrigues. 2015. "Design and Evaluation of a Unique SYBR Green Real-Time RT-PCR Assay for Quantification of Five Major Cytokines in Cattle, Sheep and Goats." BMC Veterinary Research 11 (March). https://doi.org/10.1186/s12917-015-0382-0.

Quinn, K. E., A. K. Ashley, L. P. Reynolds, A. T. Grazul-Bilska, and R. L. Ashley. 2014. "Activation of the CXCL12/CXCR4 Signaling Axis May Drive Vascularization of the Ovine Placenta." Domestic Animal Endocrinology 47 (April): 11-21. https://doi.org/10.1016/j.domaniend.2013.12.004.

Ra, Hyun-Jeong, and William C. Parks. 2007. "Control of Matrix Metalloproteinase Catalytic Activity." Matrix Biology : Journal of the International Society for Matrix Biology 26 (8): 587-96. https://doi.org/10.1016/j.matbio.2007.07.001. 
Rai-el-Balhaa, G., A. Abdullah, J. L. Pellerin, D. Thibaud, and G. Bodin. 1987. "Blastogenic Response of Peripheral Blood Lymphocytes from Multiparous Pregnant Ewes." American Journal of Reproductive Immunology and Microbiology: AJRIM 14 (4): 110-14. https://doi.org/10.1111/j.1600-0897.1987.tb00130.x.

Rainard, Pascal, Céline Riollet, Patricia Berthon, Patricia Cunha, Angélina Fromageau, Christelle Rossignol, and Florence B. Gilbert. 2008. "The Chemokine CXCL3 Is Responsible for the Constitutive Chemotactic Activity of Bovine Milk for Neutrophils." Molecular Immunology 45 (15): 4020-27. https://doi.org/10.1016/j.molimm.2008.06.010.

Rajarathnam, Krishna, Michael Schnoor, Ricardo M. Richardson, and Sudarshan Rajagopal. 2019. "How Do Chemokines Navigate Neutrophils to the Target Site: Dissecting the Structural Mechanisms and Signaling Pathways." Cellular Signalling 54 (February): 69-80. https://doi.org/10.1016/j.cellsig.2018.11.004.

Rashid, Mohammad B., Anup K. Talukder, Kazuya Kusama, Shingo Haneda, Toshiro Takedomi, Hitomi Yoshino, Satoru Moriyasu, et al. 2018. "Evidence That Interferon-Tau Secreted from Day-7 Embryo in Vivo Generates Anti-Inflammatory Immune Response in the Bovine Uterus." Biochemical and Biophysical Research Communications 500 (4): 879-84. https://doi.org/10.1016/j.bbrc.2018.04.178.

Reimers, T. J., M. B. Ullmann, and W. Hansel. 1985. "Progesterone and Prostanoid Production by Bovine Binucleate Trophoblastic Cells1." Biology of Reproduction 33 (5): 1227-36. https://doi.org/10.1095/biolreprod33.5.1227.

Retief, Francois P., and Louise Cilliers. 1998. "The Epidemic of Athens, 430 - 426 BC." South African Medical Journal 88 (1): 50-53.

Reynolds, Lawrence P., and Dale A. Redmer. 2001. "Angiogenesis in the Placenta." Biology of Reproduction 64 (4): 1033-40. https://doi.org/10.1095/biolreprod64.4.1033.

Reynolds, Lawrence, and D Redmer. 1995. "Utero-Placental Vascular Development and Placental Function." Journal of Animal Science 73 (July): 1839-51. https://doi.org/10.2527/1995.7361839x.

Rhodes, Jennifer, Andreas Hagen, Karl Hsu, Min Deng, Ting Xi Liu, A. Thomas Look, and John P. Kanki. 2005. "Interplay of Pu.1 and Gata1 Determines Myelo-Erythroid Progenitor Cell Fate in Zebrafish." Developmental Cell 8 (1): 97-108. https://doi.org/10.1016/j.devcel.2004.11.014.

Richardson, Ricardo M., Hydar Ali, Bryan C. Pridgen, Bodduluri Haribabu, and Ralph Snyderman. 1998. "Multiple Signaling Pathways of Human Interleukin-8 Receptor A: INDEPENDENT REGULATION BY PHOSPHORYLATION*." Journal of Biological Chemistry 273 (17): 10690-95. https://doi.org/10.1074/jbc.273.17.10690.

Richardson, Ricardo M., Robin J. Marjoram, Larry S. Barak, and Ralph Snyderman. 2003. "Role of the Cytoplasmic Tails of CXCR1 and CXCR2 in Mediating Leukocyte Migration, Activation, and Regulation." The Journal of Immunology 170 (6): 2904-11. https://doi.org/10.4049/jimmunol.170.6.2904.

Rifkin, Daniel B., Roberta Mazzieri, John S. Munger, Irene Noguera, and Joanne Sung. 1999. "Proteolytic Control of Growth Factor Availability." APMIS 107 (1-6): 80-85. https://doi.org/10.1111/j.16990463.1999.tb01529.x.

Roberts, R. M., S. Xie, and W. E. Trout. 1993. "Embryo-Uterine Interactions in Pigs during Week 2 of Pregnancy." Journal of Reproduction and Fertility. Supplement 48: 171-86.

Roberts, R. Michael. 2007. "Interferon-Tau, a Type 1 Interferon Involved in Maternal Recognition of Pregnancy." Cytokine \& Growth Factor Reviews, Honoring the Milstein Family Support of Interferon Research, 18 (5): 403-8. https://doi.org/10.1016/j.cytogfr.2007.06.010.

Roberts, R. Michael, James C. Cross, and Douglas W. Leaman. 1992. "Interferons as Hormones of Pregnancy." Endocrine Reviews 13 (3): 432-52. https://doi.org/10.1210/edrv-13-3-432. 
Roberts, R Michael, Jonathan A Green, and Laura C Schulz. 2016. "The Evolution of the Placenta." Reproduction (Cambridge, England) 152 (5): R179-89. https://doi.org/10.1530/REP-16-0325.

Roberts, R. Michael, Sancai Xie, and Nagappan Mathialagan. 1996. "Maternal Recognition of Pregnancy." Biology of Reproduction 54 (2): 294-302. https://doi.org/10.1095/biolreprod54.2.294.

Rodgers, J R, and R R Rich. 2013. "Antigens and Antigen Presentation." In Clinical Immunology Principles and Practice, 4th ed., 77-89. Elsevier Health Sciences.

Ruangpanit, Neeracha, John T. Price, Kenn Holmbeck, Henning Birkedal-Hansen, Volkmar Guenzler, Xinfan Huang, Danny Chan, John F. Bateman, and Erik W. Thompson. 2002. "MT1-MMPDependent and -Independent Regulation of Gelatinase A Activation in Long-Term, AscorbateTreated Fibroblast Cultures: Regulation by Fibrillar Collagen." Experimental Cell Research 272 (2): 109-18. https://doi.org/10.1006/excr.2001.5403.

Rundhaug, Joyce E. 2005. "Matrix Metalloproteinases and Angiogenesis." Journal of Cellular and Molecular Medicine 9 (2): 267-85. https://doi.org/10.1111/j.1582-4934.2005.tb00355.x.

Saad, A. M., C. Concha, and G. ÅStröm. 1989. "Alterations in Neutrophil Phagocytosis and Lymphocyte Blastogenesis in Dairy Cows Around Parturition." Journal of Veterinary Medicine, Series B 36 (110): 337-45. https://doi.org/10.1111/j.1439-0450.1989.tb00612.x.

Sakumoto, Ryosuke, Ken-Go Hayashi, Shiori Fujii, Hiroko Kanahara, Misa Hosoe, Tadashi Furusawa, and Keiichiro Kizaki. 2017. "Possible Roles of CC- and CXC-Chemokines in Regulating Bovine Endometrial Function during Early Pregnancy." International Journal of Molecular Sciences 18 (4). https://doi.org/10.3390/ijms18040742.

Salamonsen, L. A., H. Nagase, R. Suzuki, and D. E. Woolley. 1993. "Production of Matrix Metalloproteinase 1 (Interstitial Collagenase) and Matrix Metalloproteinase 2 (Gelatinase A: 72 KDa Gelatinase) by Ovine Endometrial Cells in Vitro: Different Regulation and Preferential Expression by Stromal Fibroblasts." Journal of Reproduction and Fertility 98 (2): 583-89. https://doi.org/10.1530/jrf.0.0980583.

Salamonsen, L. A., H. Nagase, and D. E. Woolley. 1991. "Production of Matrix Metalloproteinase 3 (Stromelysin) by Cultured Ovine Endometrial Cells." Journal of Cell Science 100 ( Pt 2) (October): 381-85.

- - . 1995. "Matrix Metalloproteinases and Their Tissue Inhibitors at the Ovine Trophoblast-Uterine Interface." Journal of Reproduction and Fertility. Supplement 49: 29-37.

Salcedo, Rosalba, and Joost J. Oppenheim. 2003. "Role of Chemokines in Angiogenesis: CXCL12/SDF-1 and CXCR4 Interaction, a Key Regulator of Endothelial Cell Responses." Microcirculation 10 (34): 359-70. https://doi.org/10.1038/sj.mn.7800200.

Santos, R. L., S. Zhang, R. M. Tsolis, A. J. Bäumler, and L. G. Adams. 2002. "Morphologic and Molecular Characterization of Salmonella Typhimurium Infection in Neonatal Calves." Veterinary Pathology 39 (2): 200-215. https://doi.org/10.1354/vp.39-2-200.

Sasser, R. G., Carla A. Ruder, Kristen A. Ivani, James E. Butler, and William C. Hamilton. 1986. "Detection of Pregnancy by Radioimmunoassay of a Novel Pregnancy-Specific Protein in Serum of Cows and a Profile of Serum Concentrations during Gestation1." Biology of Reproduction 35 (4): 936-42. https://doi.org/10.1095/biolreprod35.4.936.

Sato, Takashi, Takayuki Kondo, Tetsunori Fujisawa, Motoharu Seiki, and Akira Ito. 1999. "FurinIndependent Pathway of Membrane Type 1-Matrix Metalloproteinase Activation in Rabbit Dermal Fibroblasts." Journal of Biological Chemistry 274 (52): 37280-84. https://doi.org/10.1074/jbc.274.52.37280.

Sattler, Susanne. 2017. "The Role of the Immune System Beyond the Fight Against Infection." Advances in Experimental Medicine and Biology 1003: 3-14. https://doi.org/10.1007/978-3-319-576138_1. 
Scott, E. W., M. C. Simon, J. Anastasi, and H. Singh. 1994. "Requirement of Transcription Factor PU.1 in the Development of Multiple Hematopoietic Lineages." Science 265 (5178): 1573-77. https://doi.org/10.1126/science.8079170.

Seddiki, Nabila, Brigitte Santner-Nanan, Stuart G. Tangye, Stephen I. Alexander, Michael Solomon, Soon Lee, Ralph Nanan, and Barbara Fazekas de Saint Groth. 2006. "Persistence of Naive CD45RA+ Regulatory T Cells in Adult Life." Blood 107 (7): 2830-38. https://doi.org/10.1182/blood-200506-2403.

Segerson, Edward C., Hong Li, and Charles W. Talbott. 1997. "Estradiol-17 $\beta$ and Progesterone Increase Ovine Uterine Suppressor Cell Activity." Journal of Animal Science 75 (10): 2778-87. https://doi.org/10.2527/1997.75102778x.

Serrano-Pérez, B., P. J. Hansen, R. Mur-Novales, I. García-Ispierto, N. M. de Sousa, J. F. Beckers, S. Almería, and F. López-Gatius. 2016. "Crosstalk between Uterine Serpin (SERPINA14) and Pregnancy-Associated Glycoproteins at the Fetal-Maternal Interface in Pregnant Dairy Heifers Experimentally Infected with Neospora Caninum." Theriogenology 86 (3): 824-30. https://doi.org/10.1016/j.theriogenology.2016.03.003.

Sharma, Sherven, Seok-Chul Yang, Sven Hillinger, Li X Zhu, Min Huang, Raj K Batra, Jeff F Lin, Marie D Burdick, Robert M Strieter, and Steven M Dubinett. 2003. "SLC/CCL21-Mediated Anti-Tumor Responses Require IFNy, MIG/CXCL9 and IP-10/CXCL10." Molecular Cancer 2 (April): 22. https://doi.org/10.1186/1476-4598-2-22.

Shelton, K., T. J. Parkinson, M. G. Hunter, R. W. Kelly, and G. E. Lamming. 1990. "Prostaglandin E-2 as a Potential Luteotrophic Agent during Early Pregnancy in Cattle." Journal of Reproduction and Fertility 90 (1): 11-17. https://doi.org/10.1530/jrf.0.0900011.

Short, Roger V. 1969. Implantation and the Maternal Recognition of Pregnancy. John Wiley \& Sons. Silva, E., R. A. Sterry, D. Kolb, N. Mathialagan, M. F. McGrath, J. M. Ballam, and P. M. Fricke. 2007. "Accuracy of a Pregnancy-Associated Glycoprotein ELISA to Determine Pregnancy Status of Lactating Dairy Cows Twenty-Seven Days After Timed Artificial Insemination." Journal of Dairy Science 90 (10): 4612-22. https://doi.org/10.3168/jds.2007-0276.

Simintiras, Constantine A., José M. Sánchez, Michael McDonald, and Pat Lonergan. 2019a. "Progesterone Alters the Bovine Uterine Fluid Lipidome during the Period of Elongation." Reproduction 157 (4): 399-411. https://doi.org/10.1530/REP-18-0615.

Simintiras, Constantine A., José M. Sánchez, Michael McDonald, and Patrick Lonergan. 2019b. "The Influence of Progesterone on Bovine Uterine Fluid Energy, Nucleotide, Vitamin, Cofactor, Peptide, and Xenobiotic Composition during the Conceptus Elongation-Initiation Window." Scientific Reports 9 (May). https://doi.org/10.1038/s41598-019-44040-6.

Simintiras, Constantine A, José M Sánchez, Michael McDonald, and Patrick Lonergan. 2019c. "The Biochemistry Surrounding Bovine Conceptus Elongation." Biology of Reproduction 101 (2): 32837. https://doi.org/10.1093/biolre/ioz101.

Simintiras, Constantine A, José M Sánchez, Michael McDonald, Thiago Martins, Mario Binelli, and Pat Lonergan. 2019. "Biochemical Characterization of Progesterone-Induced Alterations in Bovine Uterine Fluid Amino Acid and Carbohydrate Composition during the Conceptus Elongation Window+." Biology of Reproduction 100 (3): 672-85. https://doi.org/10.1093/biolre/ioy234.

Skopets, Boris, Wen-Jun Liu, and Peter J. Hansen. 1995. "Effects of Endometrial Serpin-Like Proteins on Immune Responses in Sheep." American Journal of Reproductive Immunology 33 (1): 86-93. https://doi.org/10.1111/j.1600-0897.1995.tb01143.x.

Smith, Michael F., William A. Ricke, Leanne J. Bakke, Mark P. D. Dow, and George W. Smith. 2002. "Ovarian Tissue Remodeling: Role of Matrix Metalloproteinases and Their Inhibitors." Molecular and Cellular Endocrinology 191 (1): 45-56. https://doi.org/10.1016/S0303-7207(02)00054-0. 
Smith, Michael, Eric Mcintush, William Ricke, F Kojima, and G Smith. 1999. "Regulation of Ovarian Extracellular Matrix Remodelling by Metalloproteinases and Their Tissue Inhibitors: Effects on Follicular Development, Ovulation and Luteal Function." Journal of Reproduction and Fertility. Supplement 54 (February): 367-81. https://doi.org/10.1530/biosciprocs.4.029.

Soares, Michael J. 2004. "The Prolactin and Growth Hormone Families: Pregnancy-Specific Hormones/Cytokines at the Maternal-Fetal Interface." Reproductive Biology and Endocrinology : RB\&E 2 (July): 51. https://doi.org/10.1186/1477-7827-2-51.

Spencer, T. E, R. C Burghardt, G. A Johnson, and F. W Bazer. 2004. "Conceptus Signals for Establishment and Maintenance of Pregnancy." Animal Reproduction Science, Research and Practice III. 15th International Congress on Animal Reproduction, 82-83 (July): 537-50. https://doi.org/10.1016/j.anireprosci.2004.04.014.

Spencer, Thomas E. 2014. "Biological Roles of Uterine Glands in Pregnancy." Seminars in Reproductive Medicine 32 (5): 346-57. https://doi.org/10.1055/s-0034-1376354.

Spencer, Thomas E., Greg A. Johnson, Fuller W. Bazer, and Robert C. Burghardt. 2004. "Implantation Mechanisms: Insights from the Sheep." Reproduction 128 (6): 657-68. https://doi.org/10.1530/rep.1.00398.

Spencer, Thomas E., Andrew M. Kelleher, and Frank F. Bartol. 2019. "Development and Function of Uterine Glands in Domestic Animals." Annual Review of Animal Biosciences 7 (1): 125-47. https://doi.org/10.1146/annurev-animal-020518-115321.

Staples, L. D., R. M. Binns, and R. B. Heap. 1983. "Influence of Certain Steroids on Lymphocyte Transformation in Sheep and Goats Studied in Vitro." Journal of Endocrinology 98 (1): 55-69. https://doi.org/10.1677/joe.0.0980055.

Staples, Linton D., R. Brian Heap, David Brown, and Robert W. Marrs. 1984. "Structural Requirements for Steroid Inhibition of Sheep Lymphocyte Mitogenesis \{ulln Vitro]." Steroids 44 (5): 419-33. https://doi.org/10.1016/S0039-128X(84)80002-1.

Strieter, Robert M., Marie D. Burdick, Javier Mestas, Brigitte Gomperts, Michael P. Keane, and John A. Belperio. 2006a. "Cancer CXC Chemokine Networks and Tumour Angiogenesis." European Journal of Cancer, Cancer and inflammation, 42 (6): 768-78. https://doi.org/10.1016/j.ejca.2006.01.006.

- - . 2006b. "Cancer CXC Chemokine Networks and Tumour Angiogenesis." European Journal of Cancer, Cancer and inflammation, 42 (6): 768-78. https://doi.org/10.1016/j.ejca.2006.01.006.

Strieter, Robert M., Peter J. Polverini, Steven L. Kunkel, Douglas A. Arenberg, Marie D. Burdick, James Kasper, Judith Dzuiba, et al. 1995. "The Functional Role of the ELR Motif in CXC ChemokineMediated Angiogenesis." Journal of Biological Chemistry 270 (45): 27348-57. https://doi.org/10.1074/jbc.270.45.27348.

Strongin, Alex Y., Ivan Collier, Gregory Bannikov, Barry L. Marmer, Gregory A. Grant, and Gregory I. Goldberg. 1995. "Mechanism Of Cell Surface Activation Of 72-KDa Type IV Collagenase: ISOLATION OF THE ACTIVATED FORM OF THE MEMBRANE METALLOPROTEASE (*)." Journal of Biological Chemistry 270 (10): 5331-38. https://doi.org/10.1074/jbc.270.10.5331.

Struyf, Sofie, Paul Proost, Jean-Pierre Lenaerts, Griet Stoops, Anja Wuyts, and Jo Van Damme. 2001. "Identification of a Blood-Derived Chemoattractant for Neutrophils and Lymphocytes as a Novel CC Chemokine, Regakine-1." Blood 97 (8): 2197-2204. https://doi.org/10.1182/blood.V97.8.2197.

Sun, Chun Xiang, Marco A.O. Magalhães, and Michael Glogauer. 2007. "Rac1 and Rac2 Differentially Regulate Actin Free Barbed End Formation Downstream of the FMLP Receptor." The Journal of Cell Biology 179 (2): 239-45. https://doi.org/10.1083/jcb.200705122. 
Sun, Lizhe, Xianwei Wang, Jason Saredy, Zuyi Yuan, Xiaofeng Yang, and Hong Wang. 2020. "InnateAdaptive Immunity Interplay and Redox Regulation in Immune Response." Redox Biology 37 (October): 101759. https://doi.org/10.1016/j.redox.2020.101759.

Surveyor, G A, S J Gendler, L Pemberton, S K Das, I Chakraborty, J Julian, R A Pimental, C C Wegner, S K Dey, and D D Carson. 1995. "Expression and Steroid Hormonal Control of Muc-1 in the Mouse Uterus." Endocrinology 136 (8): 3639-47. https://doi.org/10.1210/endo.136.8.7628404.

Suzuki, K, M Lees, G F Newlands, H Nagase, and D E Woolley. 1995. "Activation of Precursors for Matrix Metalloproteinases 1 (Interstitial Collagenase) and 3 (Stromelysin) by Rat Mast-Cell Proteinases I and II." Biochemical Journal 305 (Pt 1): 301-6.

Takada, Kensuke, and Stephen C. Jameson. 2009. "Naive T Cell Homeostasis: From Awareness of Space to a Sense of Place." Nature Reviews Immunology 9 (12): 823-32. https://doi.org/10.1038/nri2657.

Takada, Yoshikazu, Xiaojing Ye, and Scott Simon. 2007. "The Integrins." Genome Biology 8 (5): 215. https://doi.org/10.1186/gb-2007-8-5-215.

Talukder, Anup K., Mohamed S. Yousef, Mohammad B. Rashid, Kensuke Awai, Tomas J. Acosta, Takashi SHIMIZU, Kiyoshi OKUDA, Masayuki SHIMADA, Kazuhiko IMAKAWA, and Akio MIYAMOTO. 2017. "Bovine Embryo Induces an Anti-Inflammatory Response in Uterine Epithelial Cells and Immune Cells in Vitro: Possible Involvement of Interferon Tau as an Intermediator." The Journal of Reproduction and Development 63 (4): 425-34. https://doi.org/10.1262/jrd.2017-056.

Taubert, Anja, Horst Zahner, and Carlos Hermosilla. 2006. "Dynamics of Transcription of Immunomodulatory Genes in Endothelial Cells Infected with Different Coccidian Parasites." Veterinary Parasitology 142 (3): 214-22. https://doi.org/10.1016/j.vetpar.2006.07.021.

Tekin, Şaban, and Peter J. Hansen. 2004. "Regulation of Numbers of Macrophages in the Endometrium of the Sheep by Systemic Effects of Pregnancy, Local Presence of the Conceptus, and Progesterone." American Journal of Reproductive Immunology 51 (1): 56-62. https://doi.org/10.1046/j.8755-8920.2003.00125.x.

Telugu, Bhanu Prakash V. L., and Jonathan A. Green. 2008. "Characterization of the Peptidase Activity of Recombinant Porcine Pregnancy-Associated Glycoprotein-2." The Journal of Biochemistry 144 (6): 725-32. https://doi.org/10.1093/jb/mvn127.

Telugu, Bhanu Prakash V.L., Mark O. Palmier, Steven R. Van Doren, and Jonathan A. Green. 2010. "An Examination of the Proteolytic Activity for Bovine Pregnancy-Associated Glycoprotein 2 and 12." Biological Chemistry 391 (2-3): 259-70. https://doi.org/10.1515/BC.2010.016.

Telugu, Bhanu Prakash VL, Angela M Walker, and Jonathan A Green. 2009. "Characterization of the Bovine Pregnancy-Associated Glycoprotein Gene Family - Analysis of Gene Sequences, Regulatory Regions within the Promoter and Expression of Selected Genes." BMC Genomics 10 (April): 185. https://doi.org/10.1186/1471-2164-10-185.

Thathiah, Amantha, and Daniel D. Carson. 2002. "Mucins and Blastocyst Attachment." Reviews in Endocrine and Metabolic Disorders 3 (2): 87-96. https://doi.org/10.1023/A:1015446626671.

Thordarson, G., G. H. McDowell, S. V. Smith, S. Iley, and I. A. Forsyth. 1987. "Effects of Continuous Intravenous Infusion of an Ovine Placental Extract Enriched in Placental Lactogen on Plasma Hormones, Metabolites and Metabolite Biokinetics in Non-Pregnant Sheep." Journal of Endocrinology 113 (2): 277-83. https://doi.org/10.1677/joe.0.1130277.

Touzard, Eve, Pierrette Reinaud, Olivier Dubois, Catherine Guyader-Joly, Patrice Humblot, Claire Ponsart, and Gilles Charpigny. 2013. "Specific Expression Patterns and Cell Distribution of Ancient and Modern PAG in Bovine Placenta during Pregnancy." Reproduction 146 (4): 347-62. https://doi.org/10.1530/REP-13-0143. 
Tsai, Shaw-Jenq, Jennifer L. Juengel, and Milo C. Wiltbank. 1997. "Hormonal Regulation of Monocyte Chemoattractant Protein-1 Messenger Ribonucleic Acid Expression in Corpora Lutea." Endocrinology 138 (10): 4517-20. https://doi.org/10.1210/endo.138.10.5577.

Tsuda, Yasuhiro, Hitoshi Takahashi, Makiko Kobayashi, Toshiaki Hanafusa, David N. Herndon, and Fujio Suzuki. 2004. "Three Different Neutrophil Subsets Exhibited in Mice with Different Susceptibilities to Infection by Methicillin-Resistant Staphylococcus Aureus." Immunity 21 (2): 215-26. https://doi.org/10.1016/j.immuni.2004.07.006.

Uekita, T., K. Yamanouchi, H. Sato, H. Tojo, M. Seiki, and C. Tachi. 2004. "Expression and Localization of Matrix Metalloproteinases (MT1-MMP, MMP-2) and Tissue Inhibitor of Metalloproteinase-2 (TIMP-2) during Synepitheliochorial Placentation of Goats (Capra Hircus)." Placenta 25 (10): 810-19. https://doi.org/10.1016/j.placenta.2004.03.007.

Ujioka, Takeshi, Akihiro Matsukawa, Nobuyuki Tanaka, Kohei Matsuura, Masaru Yoshinaga, and Hitoshi Okamura. 1998. "Interleukin-8 As an Essential Factor in the Human Chorionic GonadotropinInduced Rabbit Ovulatory Process: Interleukin-8 Induces Neutrophil Accumulation and Activation in Ovulation1." Biology of Reproduction 58 (2): 526-30. https://doi.org/10.1095/biolreprod58.2.526.

Velázquez, M. M. L., M. B. Peralta, E. Angeli, A. F. Stassi, N. C. Gareis, L. Durante, S. Cainelli, N. R. Salvetti, F. Rey, and H. H. Ortega. 2019. "Immune Status during Postpartum, Peri-Implantation and Early Pregnancy in Cattle: An Updated View." Animal Reproduction Science 206 (July): 1-10. https://doi.org/10.1016/j.anireprosci.2019.05.010.

Viana, Gregorio E. N., Virginia M. Pereira, Kinulpe Honorato-Sampaio, Cleida A. Oliveira, Robson A. S. Santos, and Adelina M. Reis. 2011. "Angiotensin-(1-7) Induces Ovulation and Steroidogenesis in Perfused Rabbit Ovaries." Experimental Physiology 96 (9): 957-65. https://doi.org/10.1113/expphysiol.2011.058453.

Vinijsanun, A., and L. Martin. 1990. "Effects of Progesterone Antagonists RU486 and ZK98734 on Embryo Transport, Development and Implantation in Laboratory Mice." Reproduction, Fertility, and Development 2 (6): 713-27. https://doi.org/10.1071/rd9900713.

Walker, Caroline G, Susanne Meier, Murray D Mitchell, John R Roche, and Mathew Littlejohn. 2009. "Evaluation of Real-Time PCR Endogenous Control Genes for Analysis of Gene Expression in Bovine Endometrium." BMC Molecular Biology 10 (November): 100. https://doi.org/10.1186/1471-2199-10-100.

Wallace, J. M., R. P. Aitken, M. A. Cheyne, and P. Humblot. 1997. "Pregnancy-Specific Protein B and Progesterone Concentrations in Relation to Nutritional Regimen, Placental Mass and Pregnancy Outcome in Growing Adolescent Ewes Carrying Singleton Fetuses." Journal of Reproduction and Fertility 109 (1): 53-58. https://doi.org/10.1530/jrf.0.1090053.

Wallace, Rhianna M., Mariah L. Hart, Tina E. Egen, Amanda Schmelzle, Michael F. Smith, Ky G. Pohler, and Jonathan A. Green. 2019. "Bovine Pregnancy Associated Glycoproteins Can Alter Selected Transcripts in Bovine Endometrial Explants." Theriogenology 131 (June): 123-32. https://doi.org/10.1016/j.theriogenology.2019.03.026.

Wallace, Rhianna M., Ky G. Pohler, Michael F. Smith, and Jonathan A. Green. 2015. "Placental PAGs: Gene Origins, Expression Patterns, and Use as Markers of Pregnancy." Reproduction 149 (3): R115-26. https://doi.org/10.1530/REP-14-0485.

Walter, I., and A. Boos. 2001. "Matrix Metalloproteinases (MMP-2 and MMP-9) and Tissue Inhibitor-2 of Matrix Metalloproteinases (TIMP-2) in the Placenta and Interplacental Uterine Wall in Normal Cows and in Cattle with Retention of Fetal Membranes." Placenta 22 (5): 473-83. https://doi.org/10.1053/plac.2001.0633.

Wang, Q, T Stacy, M Binder, M Marin-Padilla, A H Sharpe, and N A Speck. 1996. "Disruption of the Cbfa2 Gene Causes Necrosis and Hemorrhaging in the Central Nervous System and Blocks Definitive 
Hematopoiesis." Proceedings of the National Academy of Sciences of the United States of America 93 (8): 3444-49.

Wang, Zhiping, Ruth Juttermann, and Paul D. Soloway. 2000. "TIMP-2 Is Required for Efficient Activation of ProMMP-2 in Vivo." The Journal of Biological Chemistry 275 (34): 26411-15. https://doi.org/10.1074/jbc.M001270200.

Wango, E O, F B Wooding, and R B Heap. 1990. "The Role of Trophoblastic Binucleate Cells in Implantation in the Goat: A Morphological Study." Journal of Anatomy 171 (August): 241-57.

Watanabe, Norihiko, Yi-Hong Wang, Heung Kyu Lee, Tomoki Ito, Yui-Hsi Wang, Wei Cao, and Yong-Jun Liu. 2005. "Hassall's Corpuscles Instruct Dendritic Cells to Induce CD4+CD25+ Regulatory T Cells in Human Thymus." Nature 436 (7054): 1181-85. https://doi.org/10.1038/nature03886.

Webster, Nicole L., and Suzanne M. Crowe. 2006. "Matrix Metalloproteinases, Their Production by Monocytes and Macrophages and Their Potential Role in HIV-Related Diseases." Journal of Leukocyte Biology 80 (5): 1052-66. https://doi.org/10.1189/jlb.0306152.

Weems, Y. S., L. Kim, V. Humphreys, V. Tsuda, R. Blankfein, A. Wong, and C. W. Weems. 2007. "Effect of Luteinizing Hormone (LH), Pregnancy-Specific Protein B (PSPB), or Arachidonic Acid (AA) on Secretion of Progesterone and Prostaglandins (PG) E (PGE; PGE1 and PGE2) and F2 $\alpha$ (PGF2 $\alpha$ ) by Ovine Corpora Lutea of the Estrous Cycle or Pregnancy in Vitro." Prostaglandins \& Other Lipid Mediators 84 (3): 163-73. https://doi.org/10.1016/j.prostaglandins.2007.08.002.

Weems, Y. S., L. Kim, V. Humphreys, V. Tsuda, and C. W. Weems. 2003. "Effect of Luteinizing Hormone (LH), Pregnancy Specific Protein B (PSPB), or Arachidonic Acid (AA) on Ovine Endometrium of the Estrous Cycle or Placental Secretion of Prostaglandins E2 (PGE2) and F2 $\alpha$ (PGF2 $\alpha$ ) and Progesterone in Vitro." Prostaglandins \& Other Lipid Mediators 71 (1): 55-73. https://doi.org/10.1016/S0090-6980(03)00004-2.

Weems, Y. S., M. A. Lammoglia, H. R. Vera-Avila, R. D. Randel, R. G. Sasser, and C. W. Weems. 1998. "Effects of Luteinizing Hormone (LH), PGE2, 8-Epi-PGE1, 8-Epi-PGF2 $\alpha$, Trichosanthin and Pregnancy Specific Protein B (PSPB) on Secretion of Prostaglandin (PG) E (PGE) or F2 $\alpha$ (PGF2 $\alpha$ ) In Vitro by Corpora Lutea (CL) From Nonpregnant and Pregnant Cows." Prostaglandins \& Other Lipid Mediators 55 (5): 359-76. https://doi.org/10.1016/S0090-6980(98)00030-6.

Wennerberg, K., L. Lohikangas, D Gullberg, M Pfaff, S. Johansson, and R Fässler. 1996. "Beta 1 IntegrinDependent and -Independent Polymerization of Fibronectin." The Journal of Cell Biology 132 (1): 227-38.

Westrich, Joseph A., Daniel W. Vermeer, Paul L. Colbert, William C. Spanos, and Dohun Pyeon. 2020. "The Multifarious Roles of the Chemokine CXCL14 in Cancer Progression and Immune Responses." Molecular Carcinogenesis 59 (7): 794-806. https://doi.org/10.1002/mc.23188.

Widdison, S., and T. J. Coffey. 2011. "Cattle and Chemokines: Evidence for Species-Specific Evolution of the Bovine Chemokine System." Animal Genetics 42 (4): 341-53. https://doi.org/10.1111/j.1365-2052.2011.02200.x.

Wooding, F. B. 1982a. "Structure and Function of Placental Binucleate ('giant') Cells." Bibliotheca Anatomica, no. 22: 134-39.

- - . 1982b. "The Role of the Binucleate Cell in Ruminant Placental Structure." Journal of Reproduction and Fertility. Supplement 31 (November): 31-39.

- - . 1983. "Frequency and Localization of Binucleate Cells in the Placentomes of Ruminants." Placenta 4 Spec No: 527-39.

- - . 1984. "Role of Binucleate Cells in Fetomaternal Cell Fusion at Implantation in the Sheep." The American Journal of Anatomy 170 (2): 233-50. https://doi.org/10.1002/aja.1001700208.

Wooding, F. B., A. P. Flint, R. B. Heap, G. Morgan, H. L. Buttle, and I. R. Young. 1986. "Control of Binucleate Cell Migration in the Placenta of Sheep and Goats." Journal of Reproduction and Fertility 76 (2): 499-512. https://doi.org/10.1530/jrf.0.0760499. 
Wooding, F. B., G. Morgan, and C. L. Adam. 1997. "Structure and Function in the Ruminant Synepitheliochorial Placenta: Central Role of the Trophoblast Binucleate Cell in Deer." Microscopy Research and Technique 38 (1-2): 88-99. https://doi.org/10.1002/(SICI)10970029(19970701/15)38:1/2<88::AID-JEMT10>3.0.CO;2-A.

Wooding, F. B., G. Morgan, M. R. Brandon, and S. Camous. 1994. “Membrane Dynamics during Migration of Placental Cells through Trophectodermal Tight Junctions in Sheep and Goats." Cell and Tissue Research 276 (2): 387-97. https://doi.org/10.1007/BF00306124.

Wooding, F. B., G. Morgan, S. Monaghan, M. Hamon, and R. B. Heap. 1996. "Functional Specialization in the Ruminant Placenta: Evidence for Two Populations of Fetal Binucleate Cells of Different Selective Synthetic Capacity." Placenta 17 (1): 75-86. https://doi.org/10.1016/s01434004(05)80646-0.

Wooding, F. B. P. 1992. "The Synepitheliochorial Placenta of Ruminants: Binucleate Cell Fusions and Hormone Production." Placenta 13 (2): 101-13. https://doi.org/10.1016/0143-4004(92)90025O.

Wooding, F. B. P., and A. P. F. Flint. 1994. "Placentation." In G. E. Lamming (Ed) Marshall's Physiology of Reproduction, 3:233-460. London: Chapman and Hall.

Wooding, F. B. P., R. M. Roberts, and J. A. Green. 2005. "Light and Electron Microscope Immunocytochemical Studies of the Distribution of Pregnancy Associated Glycoproteins (PAGs) throughout Pregnancy in the Cow: Possible Functional Implications." Placenta 26 (10): 807-27. https://doi.org/10.1016/j.placenta.2004.10.014.

Wooding, F B, L D Staples, and M A Peacock. 1982. "Structure of Trophoblast Papillae on the Sheep Conceptus at Implantation." Journal of Anatomy 134 (Pt 3): 507-16.

Wooding, F. B., and D. C. Wathes. 1980. "Binucleate Cell Migration in the Bovine Placentome" 59 (2): 425-30. https://doi.org/10.1530/jrf.0.0590425.

Wooding, Peter, and Graham Burton. 2008. Comparative Placentation: Structures, Functions and Evolution. Springer Science \& Business Media.

Xie, S C, B G Low, R J Nagel, K K Kramer, R V Anthony, A P Zoli, J F Beckers, and R M Roberts. 1991. "Identification of the Major Pregnancy-Specific Antigens of Cattle and Sheep as Inactive Members of the Aspartic Proteinase Family." Proceedings of the National Academy of Sciences of the United States of America 88 (22): 10247-51.

Xie, S., J. Green, B. Bao, J. F. Beckers, K. E. Valdez, L. Hakami, and R. M. Roberts. 1997. "Multiple Pregnancy-Associated Glycoproteins Are Secreted by Day 100 Ovine Placental Tissue." Biology of Reproduction 57 (6): 1384-93.

Xie, Sancai, Jonathan Green, James B. Bixby, Bozena Szafranska, James C. DeMartini, Steven Hecht, and R. Michael Roberts. 1997. "The Diversity and Evolutionary Relationships of the PregnancyAssociated Glycoproteins, an Aspartic Proteinase Subfamily Consisting of Many TrophoblastExpressed Genes." Proceedings of the National Academy of Sciences of the United States of America 94 (24): 12809-16.

Xie, Sancai, Boon G. Low, Robert J. Nagel, Jean-François Beckers, and R. Michael Roberts. 1994. “A Novel Glycoprotein of the Aspartic Proteinase Gene Family Expressed in Bovine Placental Trophectoderm." Biology of Reproduction 51 (6): 1145-53. https://doi.org/10.1095/biolreprod51.6.1145.

Xu, Xiuxiu, Yonggang Zhou, and Haiming Wei. 2020. "Roles of HLA-G in the Maternal-Fetal Immune Microenvironment." Frontiers in Immunology 11 (October): 592010. https://doi.org/10.3389/fimmu.2020.592010.

Xu, Xuehua, Nidhi Gera, Hongyan Li, Michelle Yun, Liyong Zhang, Youhong Wang, Q. Jane Wang, and Tian Jin. 2015. “GPCR-Mediated PLC $\beta$ /PKC $\beta / P K D$ Signaling Pathway Regulates the Cofilin 
Phosphatase Slingshot 2 in Neutrophil Chemotaxis." Molecular Biology of the Cell 26 (5): 874-86. https://doi.org/10.1091/mbc.E14-05-0982.

Zhang, Shuping, L. Garry Adams, Jairo Nunes, Sangeeta Khare, Renée M. Tsolis, and Andreas J. Bäumler. 2003. "Secreted Effector Proteins of Salmonella Enterica Serotype Typhimurium Elicit HostSpecific Chemokine Profiles in Animal Models of Typhoid Fever and Enterocolitis." Infection and Immunity 71 (8): 4795-4803. https://doi.org/10.1128/IAI.71.8.4795-4803.2003.

Ziecik, A. J. 2002. "Old, New and the Newest Concepts of Inhibition of Luteolysis during Early Pregnancy in Pig." Domestic Animal Endocrinology, Fourth International Conference on Farm Animal Endocrinology, 23 (1): 265-75. https://doi.org/10.1016/S0739-7240(02)00162-5.

Zoli, A P, Jean-Francois Beckers, Patricia Wouters-Ballman, Jean Closset, Paul Falmagne, and Francis Ectors. 1991. "Purification and Characterization of a Bovine Pregnancy-Associated Glycoprotein." Biology of Reproduction 45 (1): 1-10. https://doi.org/10.1095/biolreprod45.1.1.

Zoli, Andre Pagnah, Louis A. Guilbault, Philippe Delahaut, Washington Benitez Ortiz, and Jean-François Beckers. 1992. "Radioimmunoassay of a Bovine Pregnancy-Associated Glycoprotein in Serum: Its Application for Pregnancy Diagnosis." Biology of Reproduction 46 (1): 83-92. https://doi.org/10.1095/biolreprod46.1.83. 